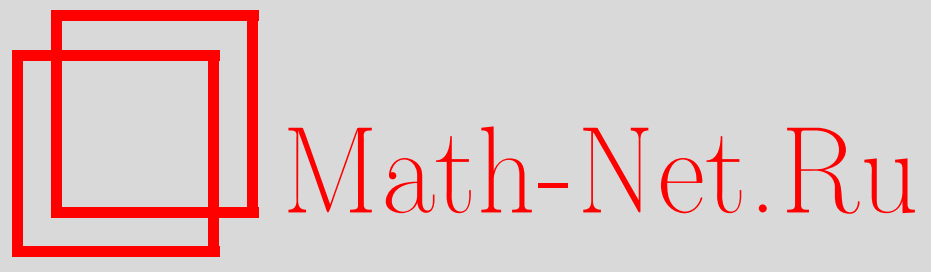

И. Б. Пенков, А. С. Тихомиров, Тривиальность векторных расслоений на скрученных инд-грассманианах, $M a-$ тем. сб., 2011, том 202, номер 1, 65-104

DOI: https://doi.org/10.4213/sm7599

Использование Общероссийского математического портала Math-Net.Ru подразумевает, что вы прочитали и согласны с пользовательским соглашением http://www . mathnet.ru/rus/agreement

Параметры загрузки:

IP : 54.162 .127 .20

26 апреля 2023 г., $17: 54: 30$

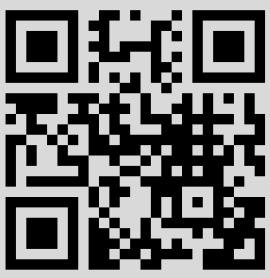




\author{
И. Б. Пенков, А.С. Тихомиров
}

\title{
Тривиальность векторных расслоений на скрученных инд-грассманианах
}

\begin{abstract}
Скрученными инд-грассманианами называются инд-многообразия $\mathbf{G}$, определяемые как прямые пределы грассманианов $G\left(i_{m}, V^{n_{m}}\right)$ для $m \in$ $\mathbb{Z}_{>0}$ при вложениях степени больше единицы. И. Дониным и И. Пенковым (2003 г.) была высказана гипотеза о том, что всякое векторное расслоение конечного ранга на скрученном инд-грассманиане тривиально. Мы доказываем эту гипотезу.

Библиография: 16 названий.
\end{abstract}

Ключевые слова: инд-многообразие, скрученный инд-грассманиан, векторное расслоение.

\section{§1. Введение и формулировка основного результата}

1.1. Инд-грассманиан $\mathbf{G}=\underline{\lim } G\left(i_{m}, V^{n_{m}}\right)$ есть инд-многообразие, определяемое как прямой предел цепочки вложений

$$
G\left(i_{1}, V^{n_{1}}\right) \stackrel{\varphi_{1}}{\hookrightarrow} G\left(i_{2}, V^{n_{2}}\right) \stackrel{\varphi_{2}}{\longrightarrow} \cdots \stackrel{\varphi_{m-1}}{\hookrightarrow} G\left(i_{m}, V^{n_{m}}\right) \stackrel{\varphi_{m}}{\longrightarrow} \cdots
$$

где через $G(i, V)$ обозначается грассманиан $i$-мерных подпространств в конечномерном векторном пространстве $V$. Каждое вложение $\varphi_{m}$ имеет корректно определенную степень $\operatorname{deg} \varphi_{m}$, и инд-грассманиан $\mathbf{G}$ называется скрученнъцм, если $\operatorname{deg} \varphi_{m}>1$ для бесконечно большого числа значений $m$. Векторное расслоение $\mathbf{E}$ ранга $\mathbf{r} \in \mathbb{Z}_{>0}$ на $\mathbf{G}$ определяется как обратный предел $\underset{\lim }{\longleftarrow} E_{m}$ обратной системы векторных расслоений $E_{m}$ ранга $\mathbf{r}$ на $G\left(i_{m}, V^{n_{m}}\right)$ (т.е. системы векторных расслоений $E_{m}$ с фиксированными изоморфизмами $\left.\psi_{m}: E_{m} \cong \varphi_{m}^{*} E_{m+1}\right)$.

В частном случае, когда $i_{m}=1$ и $\operatorname{deg} \varphi_{m}=1$ для всех $m$, изучение векторных расслоений конечного ранга на инд-грассманианах восходит к В. Барту и А. Ван де Вену (см. [1]) и А.Н. Тюрину (см. [2]). В этом случае G есть бесконечномерное проективное пространство $\mathbb{P}^{\infty}$, и замечательная теорема Барта-Ван де Вена-Тюрина гласит, что всякое векторное расслоение конечного ранга на $\mathbb{P}^{\infty}$ изоморфно прямой сумме линейных расслоений. Исторически она впервые явилась отражением следующего общего феномена, по-видимому, имеющего место для инд-многообразий, определяемых последовательностями вложений $(1.1)$ с заменой $G\left(i_{m}, V_{m}\right)$ на произвольные флаговые многообразия: во всех известных случаях ограничение векторного расслоения конечного ранга

Работа выполнена при частичной поддержке Немецкого исследовательского общества (грант № DFG PE980/3-1). 
с инд-многообразия на достаточно большое конечномерное флаговое подмногообразие эквивариантно. Примерно в то же время это явление было обнаружено и в работах Е. Сато, который дал независимое доказательство теоремы Барта-Ван де Вена-Тюрина (см. [3]). Вскоре после этого Сато доказал более общий результат, относящийся к инд-грассманиану $\mathbf{G}(i, V) i$-мерных подпространств в счетномерном векторном пространстве $V$ (см. [4]).

Сравнительно недавно это направление возобновилось в работах [5]-[7]. В частности, в [7] была высказана общая гипотеза о векторных расслоениях конечного ранга на скрученных инд-грассманианах. В действительности, как показано авторами в [7], если инд-грассманиан $\mathbf{G}$ не является скрученным (что равносильно тому, что $\operatorname{deg} \varphi_{m}=1$ для всех $m$ ), эта гипотеза является более или менее прямым следствием результата Сато. При этом остается открытым случай скрученного инд-грассманиана $\mathbf{G}$, в котором гипотеза гласит, что векторное расслоение конечного ранга на $\mathbf{G}$ тривиально. До настоящего времени гипотеза была доказана лишь в следующих трех случаях: для расслоений ранга 2 на произвольном скрученном инд-грассманиане (см. [7]); для расслоений конечного ранга на произвольном скрученном проективном инд-пространстве (скрученное проективное инд-пространство определяется последовательностью (1.1) с $i_{m}=1$ и $\operatorname{deg} \varphi_{m}>1$ для всех $m$; см. [5]); для произвольных расслоений конечного ранга на некоторых специальных скрученных инд-грассманианах (для которых вложения $\varphi_{m}$ являются скрученными расширениями в смысле [5]).

В настоящей статье мы доказываем данную гипотезу, т.е. устанавливаем следующую теорему.

Теорема 1. Векторное расслоение конечного ранга $\mathbf{E}=\lim _{\longleftarrow} E_{m}$ на произвольном скрученном инд-грассманиане $\mathbf{G}=\underset{\lim }{\longrightarrow}\left(i_{m}, V^{n_{m}}\right)$ тривиально.

1.2. Дадим краткое описание основных элементов доказательства теоремы 1. Во-первых, без ограничения общности мы можем считать, что расслоение $\mathbf{E}$ самодвойственно. При необходимости этого можно добиться заменой $\mathbf{E}$ на $\mathscr{E} n d \mathbf{E}$. Конечная цель доказательства состоит в построении для больших $m$ подпучков $\mathscr{F}_{m}$ в векторных расслоениях $E_{m}$ с $c_{1}\left(\mathscr{F}_{m}\right)>0$ в предположении, что $E_{m}$ нетривиальны. Это предположение затем легко приводит к противоречию, поскольку скрученность инд-грассманиана $\mathbf{G}$ и бесконечная продолжаемость расслоений $E_{m}$ влекут бесконечный рост класса $c_{1}\left(\mathscr{F}_{m}\right)$. Общая идея этой конструкции восходит к Барту, Ван де Вену и Тюрину для случая $\mathbb{P}^{\infty}$.

Конструкция подпучков $\mathscr{F}_{m}$ представляет собой комбинацию нескольких идей и основана на изучении многообразия прямых максимального подскока векторного расслоения $\mathbf{E}$. В нашем случае исследуется многообразие прямых максимального подскока векторного расслоения $E_{m}$ на грассманиане $G\left(i_{m}, V^{n_{m}}\right)$. Мы сводим задачу к изучению аналогичного многообразия для проективного пространства с помощью бирационального изоморфизма между грассманианом $G\left(i_{m}, V^{n_{m}}\right)$ и расслоенным пространством $X_{m}$ со слоем проективное пространство. Ключевым результатом в этой связи является существование универсальных оценок сверху на степень и коразмерность многообразия прямых максимального подскока векторного расслоения, проходящих через точку в проективном пространстве. 
Статья построена следующим образом. В 33 изучаются многообразия ограниченной степени и коразмерности в проективном пространстве растущей размерности. Основной результат здесь состоит в том, что любые две точки такого многообразия могут быть соединены цепочкой проективных подпространств растущей размерности. Этот результат близок по духу классическому результату А. Предонцана и включен в настоящую статью ввиду отсутствия подходящей ссылки.

В $\S 4$ мы даем достаточное условие на целое число $m$, при котором данное векторное расслоение $E$ на $\mathbb{P}^{n}$ является $m$-регулярным в смысле МамфордаКастельнуово, т.е. таким, что $H^{i}(E(m-i))=0$ для $i \geqslant 1$. Это условие на $m$ необходимо для получения оценки на степень многообразия прямых максимального подскока векторного расслоения на проективном пространстве, проходящих через точку, которую мы даем в §5. Эта оценка (см. теорему 7) дается в терминах ранга расслоения, его второго класса Черна, максимального подскока и размерности проективного пространства в предположении, что первый класс Черна расслоения равен нулю.

Конструкции подпучка $\mathscr{F}_{m}$ расслоения $E_{m}$, где $\mathbf{E}=\lim _{\longleftarrow} E_{m}$ есть самодвойственное векторное расслоение на $\mathbf{G}$, посвящен $\S 6$. Здесь мы заменяем $G\left(i_{m}, V^{n_{m}}\right)$ расслоенным пространством $X_{m}$, к слоям которого применяются предыдущие результаты о векторных расслоениях на проективных пространствах. Конструкция пучка $\mathscr{F}_{m}$ затем быстро приводит к противоречию с предположением о нетривиальности расслоения $E_{m}$, как было объяснено выше.

1.3. Приведем простой пример скрученного инд-грассманиана, для которого наша теорема дает нетривиальное утверждение. В этом примере скрученный инд-грассманиан возникает естественным образом как однородное пространство локально линейной инд-группы. Различные дальнейшие примеры скрученных инд-грассманианов можно найти в работах [5] и [7].

Интересной инд-группой является инд-группа $\mathbf{S L}(n, \operatorname{Adj})$. Зафиксируем $n$ и рассмотрим вложение

$$
\mathrm{SL}(n) \rightarrow \mathrm{SL}\left(n^{2}-1\right),
$$

определяемое условием, чтобы естественное представление группы $\operatorname{SL}\left(n^{2}-1\right)$ ограничивалось до представления, изоморфного присоединенному представлению подгруппы $\mathrm{SL}(n)$. Полагая $\mathscr{G}_{1}:=\mathrm{SL}(n), \mathscr{G}_{2}:=\mathrm{SL}\left(n^{2}-1\right)$ и итерируя эту конструкцию, получаем инд-группу $\mathbf{S L}(n, \mathrm{Adj})$ как прямой предел $\underset{\lim }{\longrightarrow} \mathscr{G}_{m}$. Зафиксируем подпространство $V_{1} \subset \mathbb{C}^{n}$. Тогда $V_{2}:=V_{1} \otimes\left(\mathbb{C}^{n} / V_{1}\right)^{\vee} \overrightarrow{\text { где }^{\vee}}$ обозначает двойственное пространство) является корректно определенным подпространством присоединенного представления группы $\mathscr{G}_{2}$. Итерирование этой последней конструкции дает подпространство $V_{m}$ естественного представления группы $\mathscr{G}_{m}$ для каждого $m$. Стабилизаторы $\mathscr{P}_{m} \subset \mathscr{G}_{m}$ пространств $V_{m}$ образуют прямую систему параболических подгрупп $\mathscr{P}_{1} \subset \mathscr{P}_{2} \subset \cdots$ со свойством $\mathscr{P}_{m} \cap \mathscr{G}_{m-1}=\mathscr{P}_{m-1}$. Это определяет замкнутые вложения

$$
\zeta_{m-1}: \mathscr{G}_{m-1} / \mathscr{P}_{m-1} \hookrightarrow \mathscr{G}_{m} / \mathscr{P}_{m}
$$

и, следовательно, инд-многообразие $\lim \mathscr{G}_{m} / \mathscr{P}_{m}$. Поскольку каждое многообразие $\mathscr{G}_{m} / \mathscr{P}_{m}$ есть грассманиан, $\overrightarrow{\lim } \mathscr{G}_{m} / \mathscr{P}_{m}$ является инд-грассманианом. Более того, ограничение на подмногообразие $\mathscr{G}_{m-1} / \mathscr{P}_{m-1}$ тавтологического 
расслоения на грассманиане $\mathscr{G}_{m} / \mathscr{P}_{m}$ изоморфно кокасательному расслоению грассманиана $\mathscr{G}_{m-1} / \mathscr{P}_{m-1}$. Это показывает, что степень вложения $\zeta_{m}$ равна размерности естественного представления группы $\mathscr{G}_{m}$. Тем самым, $\lim \mathscr{G}_{m} / \mathscr{P}_{m}$ есть скрученный инд-грассманиан. Простое упражнение показывает, что индгруппа $\lim \mathscr{P}_{m}$ не имеет нетривиальных конечномерных представлений. Поэтому $\underset{\lim }{\longrightarrow} \overrightarrow{\mathscr{G}_{m}} / \mathscr{P}_{m}=\mathbf{S L}(n, \operatorname{Adj}) / \lim _{m} \mathscr{P}_{m}$ не допускает нетривиальных $\mathbf{S L}(n, \operatorname{Adj})$ эквивариантных векторных расслоений конечного ранга. Теорема 1, однако, дает намного более сильный результат о том, что всякое векторное расслоение конечного ранга на $\underset{\lim }{\longrightarrow} \mathscr{G}_{m} / \mathscr{P}_{m}$ тривиально.

Мы благодарим Ф.Л. Зака за то, что он обратил наше внимание на работу Р. Брауна и С. Мюллера-Штаха. Мы признательны за поддержку и гостеприимство Математическому институту им. Макса Планка в Бонне, где была задумана настоящая статья. Второй автор также выражает благодарность за поддержку центру ICTS в Университете Якобса в Бремене.

\section{§ 2. Обозначения и соглашения}

2.1. Наши обозначения в основном стандартны. Основным полем является поле комплексных чисел $\mathbb{C}$. Предполагается, что все рассматриваемые векторные расслоения имеют конечный ранг. Мы отождествляем локально свободные пучки с векторными расслоениями конечного ранга и пользуемся термином “алгебраическое многообразие" или просто "многообразие" для обозначения при-

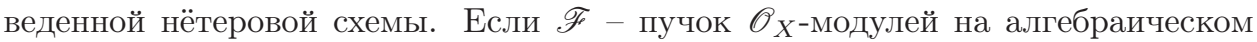
многообразии или схеме $X$, то $\mathscr{F}^{j}$ обозначает прямую сумму $j$ копий пучка $\mathscr{F}$, $H^{j}(\mathscr{F})$ обозначает $j$-ю группу когомологий пучка $\mathscr{F}, h^{j}(\mathscr{F}):=\operatorname{dim} H^{j}(\mathscr{F})$ и через $\mathscr{F} \vee$ обозначается двойственный пучок, т.е. пучок $\mathscr{F} \vee:=\mathscr{H}_{0} m_{\mathscr{O}_{X}}\left(\mathscr{F}, \mathscr{O}_{X}\right)$. Соответственно, $\mathrm{Sym}^{j}$ и $\wedge^{j}$ обозначают $j$-ю симметрическую и внешнюю степени. Если $Z \subset X$ - подмногообразие или подсхема, то через $\mathscr{I}_{Z, X}$ обозначается пучок идеалов, соответствующий $Z$. Через $\mathbb{P}(E)$ мы обозначаем проективизацию векторного расслоения $E$ (в частности, векторного пространства).

2.2. Под проективным подпространством $\mathbb{P}^{k}$ в $G(i, V)$ понимается линейно вложенное проективное подпространство, т.е. подмножество $i$-мерных подпространств $W$ в $V$ с $V_{0} \subset W \subset V_{1}$, где $V_{0} \subset V_{1}$ есть фиксированный флаг подпространств в $V$ размерностей $i-1$ и $i+k$ либо $i-k$ и $i+1$ соответственно. В частности, прямая в $G(i, V)$ определяется флагом $V_{1} \subset V_{2}$ подпространств в $V$, где $\operatorname{dim} V_{1}=i-1, \operatorname{dim} V_{2}=i+1$.

Если $C \subset X$ - гладкая неприводимая рациональная кривая на алгебраическом многообразии $X$ и $E$ - векторное расслоение на $X$, то согласно классической теореме Гротендика $\left.E\right|_{C}$ изоморфно $\bigoplus_{i} \mathscr{O}_{C}\left(\delta_{i}\right)$ для некоторых $\delta_{1} \geqslant$ $\delta_{2} \geqslant \cdots \geqslant \delta_{\mathrm{rk} E}$. Мы называем упорядоченный набор $\left(\delta_{1}, \ldots, \delta_{\mathrm{rk} E}\right)$ длины $\mathrm{rk} E$ типом расщепления расслоения $\left.E\right|_{C}$.

Пусть $E$ - векторное расслоение на $G(i, V)$. Для произвольной рациональной кривой $C$ на $G(i, V)$ рассмотрим тип расщепления $\left(\delta_{1}, \ldots, \delta_{\mathrm{rk} E}\right)$ расслоения $\left.E\right|_{C}$ и положим

$$
\begin{aligned}
\delta_{A}\left(\left.E\right|_{C}\right):= & \delta_{1}, \quad \delta_{B}\left(\left.E\right|_{C}\right):=\delta_{\operatorname{rk} E}, \quad \delta\left(\left.E\right|_{C}\right):=\delta_{A}\left(\left.E\right|_{C}\right)-\delta_{B}\left(\left.E\right|_{C}\right), \\
& \varkappa_{A}\left(\left.E\right|_{C}\right):=\max \left\{k \mid 1 \leqslant k \leqslant \operatorname{rk} E, \delta_{k}=\delta_{A}\left(\left.E\right|_{C}\right)\right\} .
\end{aligned}
$$


Далее, положим

$$
\delta_{A}(E):=\max _{l} \delta_{A}\left(\left.E\right|_{l}\right), \quad \delta_{B}(E):=\min _{l} \delta_{B}\left(\left.E\right|_{l}\right)
$$

где $l$ пробегает все прямые на $G(i, V)$,

$$
\begin{gathered}
\delta(E):=\delta_{A}(E)-\delta_{B}(E), \\
\varkappa_{A}(E):=\max \left\{\varkappa_{A}\left(\left.E\right|_{l}\right) \mid l-\text { прямая в } G(i, V) \text { такая, что } \delta_{A}\left(\left.E\right|_{l}\right)=\delta_{A}(E)\right\} .
\end{gathered}
$$

Важно отметить, что $\delta_{A}\left(\left.E\right|_{C}\right)$ и $\varkappa_{A}\left(\left.E\right|_{C}\right)$ являются полунепрерывными функциями от $C$, где $C$ принадлежит любому плоскому семейству рациональных кривых в $G(i, V)$ (см. [8; ч. III, теорема 12.8]).

2.3. Нам потребуется также одно обозначение, касающееся многочленов. Для произвольного ненулевого многочлена

$$
f\left(y_{1}, \ldots, y_{q}\right)=\sum \frac{a_{i_{1} \ldots i_{q}}}{b_{i_{1} \ldots i_{q}}} y_{1}^{i_{1}} \cdots y_{q}^{i_{q}} \in \mathbb{Q}\left[y_{1}, \ldots, y_{q}\right]
$$

с взаимно простыми коэффициентами $a_{i_{1} \ldots i_{q}} \in \mathbb{Z}$ и $b_{i_{1} \ldots i_{q}} \in \mathbb{Z}$ для всех $i_{1}, \ldots, i_{q}$ через

$$
f\left(y_{1}, \ldots, y_{q}\right)^{+} \in \mathbb{Z}\left[y_{1}, \ldots, y_{q}\right]
$$

будем обозначать многочлен $\sum a_{i_{1} \ldots i_{q}}^{2} y_{1}^{2 i_{1}} \cdots y_{q}^{2 i_{q}}$. Отметим, что

$$
-f\left(y_{1}, \ldots, y_{q}\right)^{+} \leqslant f\left(y_{1}, \ldots, y_{q}\right) \leqslant f\left(y_{1}, \ldots, y_{q}\right)^{+}
$$

для всех $y_{1}, \ldots, y_{q} \in \mathbb{Z}$.

\section{§ 3. Проективные подпространства в многообразиях ограниченной коразмерности и степени}

3.1. В этом параграфе мы доказываем, что любые две точки на многообразии ограниченной коразмерности и степени в проективном пространстве растущей размерности могут быть соединены цепочкой проективных подпространств растущей размерности, лежащих на этом многообразии. По существу это глава из теории схем Фано в духе Альтмана и Клеймана (см. [9]), и наш результат близок также к теореме Предонцана (1948 г.), современное изложение которой можно найти в [10]. Всюду в этом параграфе $d \in \mathbb{Z}_{\geqslant 2}$ постоянно, a $n \in \mathbb{Z}_{\geqslant 6}$ является переменным. Число $k \in \mathbb{Z}_{\geqslant 1}$ также является переменным и удовлетворяет неравенству

$$
n \geqslant d\left(\begin{array}{c}
k+d \\
d
\end{array}\right)+k
$$

например, можно положить $k=k(n):=[\sqrt[d+1]{n / d}]$. 
3.2. Проективные подпространства на гиперповерхности ограниченной степени и растущей размерности. Рассмотрим проективное пространство $\mathbb{P}^{n}=\mathbb{P}(V)$, где $V$ - векторное пространство размерности $n+1$. Пусть

$$
\mathbb{P}^{s}:=\left|\mathscr{O}_{\mathbb{P}^{n}}(d)\right|, \quad s=\left(\begin{array}{c}
n+d \\
d
\end{array}\right)-1,
$$

- полный линейный ряд гиперповерхностей данной степени $d$ в $\mathbb{P}^{n}$. Рассмотрим естественную диаграмму

$$
G(k+1, V) \stackrel{\widetilde{p}}{\leftarrow} \Gamma \stackrel{\widetilde{q}}{\rightarrow} \mathbb{P}^{s},
$$

где $\Gamma=\left\{\left(\mathbb{P}^{k}, H\right) \in G(k+1, V) \times \mathbb{P}^{s} \mid \mathbb{P}^{k} \subset H\right\}$ и где $G(k+1, V)$ интерпретируется как грассманиан $k$-мерных проективных подпространств в $\mathbb{P}^{n}$. Для каждой пары $\left(\mathbb{P}^{k}, H\right) \in \Gamma$ выберем однородные координаты $\left(x_{0}: x_{1}: \ldots: x_{n}\right)$ в $\mathbb{P}^{n}$ такие, что $\mathbb{P}^{k}=\left\{x_{k+1}=\cdots=x_{n}=0\right\}$. Пусть $H=\left\{f\left(x_{0}, \ldots, x_{n}\right)=0\right\}$, $f \in H^{0}\left(\mathscr{O}_{\mathbb{P}} n(d)\right)$, и

$$
\Phi_{i}\left(x_{0}, x_{1}, \ldots, x_{k}\right):=\frac{\partial f}{\partial x_{k+i}}\left(x_{0}, x_{1}, \ldots, x_{k}, 0, \ldots, 0\right), \quad 1 \leqslant i \leqslant n-k .
$$

Предположим, что гиперповерхность $H$ неособа. Тогда

$$
\bigcap_{i=1}^{n-k}\left\{\Phi_{i}\left(x_{0}, x_{1}, \ldots, x_{k}\right)=0\right\}=\varnothing
$$

и мы имеем точную последовательность нормальных расслоений на $\mathbb{P}^{k}$

$$
0 \rightarrow N_{\mathbb{P}^{k} / H} \rightarrow \mathscr{O}_{\mathbb{P}^{k}}(1)^{n-k} \stackrel{\varepsilon_{k}}{\rightarrow} \mathscr{O}_{\mathbb{P}^{k}}(d) \rightarrow 0, \quad \varepsilon_{k}:\left(L_{1}, \ldots, L_{n-k}\right) \mapsto \sum_{i=1}^{n-k} L_{i} \Phi_{i}
$$

Предположим, что $H$ является общей в том смысле, что

$$
\operatorname{Span}\left(\Phi_{1}, \ldots, \Phi_{n-k}\right)=H^{0}\left(\mathscr{O}_{\mathbb{P} k}(d-1)\right) .
$$

Тогда точная последовательность, полученная из (3.3) подкруткой на $\mathscr{O}_{\mathbb{P} k}(-1)$, индуцирует сюръективный гомоморфизм $H^{0}\left(\mathscr{O}_{\mathbb{P} k}^{n-k}\right) \rightarrow H^{0}\left(\mathscr{O}_{\mathbb{P} k}(d-1)\right)$, и легко видеть, что после обратной подкрутки на $\mathscr{O}_{\mathbb{P}^{k}}(1)$ мы получаем сюръективный гомоморфизм $h^{0}\left(\varepsilon_{k}\right): H^{0}\left(\mathscr{O}_{\mathbb{P}^{k}}(1)^{n-k}\right) \rightarrow H^{0}\left(\mathscr{O}_{\mathbb{P}^{k}}(d)\right)$. Поэтому

$h^{0}\left(N_{\mathbb{P}^{k} / H}\right)=(k+1)(n-k)-\left(\begin{array}{c}k+d \\ d\end{array}\right)>0, \quad h^{1}\left(N_{\mathbb{P}^{k} / H}\right)=h^{1}\left(N_{\mathbb{P}^{k} / H}(-1)\right)=0$

(неравенство следует из (3.1)).

Заметим, что $\widetilde{p}: \Gamma \rightarrow G(k+1, V)$ является проективным расслоением со слоем $\mathbb{P}\left(H^{0}\left(\mathscr{I}_{\mathbb{P}^{k}, \mathbb{P}^{n}}(d)\right)\right)$, а значит, Г гладко и неприводимо. Поэтому

$\operatorname{dim} \widetilde{q}^{-1}(H) \geqslant \operatorname{dim} \Gamma-s=\operatorname{dim} G(k+1, V)+\operatorname{dim} \mathbb{P}\left(H^{0}\left(\mathscr{I}_{\mathbb{P}^{k}, \mathbb{P}^{n}}(d)\right)\right)-s$

$$
=(k+1)(n-k)+\left(s-\left(\begin{array}{c}
k+d \\
d
\end{array}\right)\right)-s=(k+1)(n-k)-\left(\begin{array}{c}
k+d \\
d
\end{array}\right) .
$$


Отсюда и из (3.5) по теории деформаций получаем, что

$$
B_{H}:=\widetilde{p}\left(\widetilde{q}^{-1}(H)\right)
$$

имеет размерность

$$
\operatorname{dim} B_{H}=h^{0}\left(N_{\mathbb{P}^{k} / H}\right)=(k+1)(n-k)-\left(\begin{array}{c}
k+d \\
d
\end{array}\right)
$$

и является гладким в точке $\mathbb{P}^{k}$ для общей гладкой гиперповерхности $H \in \mathbb{P}^{s}$. Более того, проективный морфизм $\widetilde{q}$ доминантен. Поскольку образ проективного морфизма замкнут (см. [8; ч. II, §4, теорема 4.9]), это влечет сюръективность морфизма $\widetilde{q}$.

Лемма 1. Для гладкой общей (в смысле (3.4)) гиперповерхности $H \in \mathbb{P}^{s}$ $B_{H}$ есть гладкое неприводимое многообразие размерности $(k+1)(n-k)-\left(\begin{array}{c}k+d \\ d\end{array}\right)$.

ДокАЗАтельство. Гладкость $B_{H}$ следует из того факта, что $B_{H}$ есть общий слой сюръективного морфизма $\widetilde{q}: \Gamma \rightarrow \mathbb{P}^{s}$ гладких многообразий (см. [8; ч. III, $\S 10$, следствие 10.7]).

Пусть $S_{k+1}$ есть тавтологическое расслоение ранга $k+1$ на $G(k+1, V)$. Согласно [9; теорема 1.3] $B_{H}$ есть схема нулей регулярного сечения $\sigma \in H^{0}\left(T^{\vee}\right)$, где $T:=\operatorname{Sym}^{d} S_{k+1}$. Более того, имеем стандартную резольвенту Кошуля пучка $\mathscr{O}_{B_{H}}$

$$
0 \rightarrow \wedge^{\mathrm{rk} T} T \rightarrow \cdots \rightarrow \wedge^{2} T \rightarrow T \stackrel{\sigma^{\vee}}{\rightarrow} \mathscr{O}_{G(k+1, V)} \rightarrow \mathscr{O}_{B_{H}} \rightarrow 0 .
$$

Мы покажем, что

$$
H^{0}(T)=H^{j}\left(\wedge^{j} T\right)=0, \quad 1 \leqslant j \leqslant \operatorname{rk} T .
$$

Для этого рассмотрим диаграмму инцидентности

$$
G(i+1, V) \stackrel{p_{i}}{\leftarrow} \mathrm{Fl}(i, i+1, V) \stackrel{q_{i}}{\longrightarrow} G(i, V), \quad 1 \leqslant i \leqslant k .
$$

$\mathrm{Ha} \operatorname{Fl}(i, i+1, V)$ имеется точная последовательность векторных расслоений

$$
0 \rightarrow q_{i}^{*} S_{i} \stackrel{\theta_{i}}{\rightarrow} p_{i}^{*} S_{i+1} \rightarrow q_{i}^{*} \mathscr{O}_{G(i, V)}(1) \otimes p_{i}^{*} \mathscr{O}_{G(i+1, V)}(-1) \rightarrow 0,
$$

где $S_{i}$ есть тавтологическое расслоение ранга $i$ на $G(i, V)$. Ограничивая (3.10) на слой $\mathbb{P}_{y}^{n-i}:=q_{i}^{-1}(y)$ для $y \in G(i, V)$, получаем точную тройку

$$
\left.\left.0 \rightarrow q_{i}^{*} S_{i}\right|_{\mathbb{P}_{y}^{n-i}} \stackrel{\left.\theta_{i}\right|_{\mathbb{P}_{n}^{n-i}}}{\rightarrow} p_{i}^{*} S_{i+1}\right|_{\mathbb{P}_{y}^{n-i}} \rightarrow \mathscr{O}_{\mathbb{P}_{y}^{n-i}}(-1) \rightarrow 0,\left.\quad q_{i}^{*} S_{i}\right|_{\mathbb{P}_{y}^{n-i}} \simeq\left(\mathscr{O}_{\mathbb{P}_{y}^{n-i}}\right)^{i}
$$

Переходя к симметрическим степеням и полагая $s_{i}:=\left(\begin{array}{c}d+i \\ d-1\end{array}\right), t_{i}:=\left(\begin{array}{c}d+i-1 \\ d\end{array}\right)$, имеем

$$
\begin{gathered}
\left.\left.0 \rightarrow q_{i}^{*} \operatorname{Sym}^{d} S_{i}\right|_{\mathbb{P}_{y}^{n-i}} \rightarrow p_{i}^{*} \operatorname{Sym}^{d} S_{i+1}\right|_{\mathbb{P}_{y}^{n-i}} \rightarrow \bigoplus_{p=1}^{s_{i}} \mathscr{O}_{\mathbb{P}_{y}^{n-i}}\left(a_{p}\right) \rightarrow 0, \\
-d \leqslant a_{p} \leqslant-1, \quad 1 \leqslant p \leqslant s_{i}, \\
\left.q_{i}^{*} \operatorname{Sym}^{d} S_{i}\right|_{\mathbb{P}_{y}^{n-i}} \simeq\left(\mathscr{O}_{\mathbb{P}_{y}^{n-i}}\right)^{t_{i}} .
\end{gathered}
$$


Рассмотрим точные тройки

$$
\begin{gathered}
0 \rightarrow q_{i}^{*} \wedge^{j}\left(\operatorname{Sym}^{d} S_{i}\right) \stackrel{\Theta_{i j}}{\rightarrow} p_{i}^{*} \wedge^{j}\left(\operatorname{Sym}^{d} S_{i+1}\right) \rightarrow \Lambda_{i j} \rightarrow 0, \\
\Lambda_{i j}:=\operatorname{coker} \Theta_{i j}, \quad 1 \leqslant j \leqslant \operatorname{rk} T,
\end{gathered}
$$

где $\Theta_{i j}$ суть мономорфизмы, индуцируемые морфизмами $\theta_{i}$ в (3.10). После ограничения на $\mathbb{P}_{y}^{n-i}$, используя (3.11) и (3.12), получаем

$$
\left.\Lambda_{i j}\right|_{\mathbb{P}_{y}^{n-i}} \simeq \bigoplus_{q=1}^{u_{i j}} \mathscr{O}_{\mathbb{P}_{y}^{n-i}}\left(b_{q}\right), \quad-j d \leqslant b_{q} \leqslant-1, \quad 1 \leqslant q \leqslant u_{i j}
$$

где $u_{i j}:=\left(\begin{array}{c}s_{i}+t_{i} \\ j\end{array}\right)-\left(\begin{array}{c}t_{i} \\ j\end{array}\right)$. Ключевое наблюдение состоит в том, что из (3.1) и (3.14) вытекают равенства

$$
H^{a}\left(\left.\Lambda_{i j}\right|_{\mathbb{P}_{y}^{n-i}}\right)=0, \quad a \geqslant 0, \quad 1 \leqslant j \leqslant \operatorname{rk} T, \quad 1 \leqslant i \leqslant k .
$$

Отсюда следует, что спектральная последовательность Лере

$$
E_{2}^{a a^{\prime}}=H^{a}\left(R^{a^{\prime}} q_{i *} \Lambda_{i j}\right) \quad \Longrightarrow \quad H^{\cdot}\left(\Lambda_{i j}\right)
$$

вырождается и потому влечет равенства

$$
H^{a}\left(\Lambda_{i j}\right)=0, \quad a \geqslant 0, \quad 1 \leqslant j \leqslant \operatorname{rk} T, \quad 1 \leqslant i \leqslant k .
$$

Поскольку (как хорошо известно)

$$
\begin{aligned}
H^{a}\left(\wedge^{j}\left(\operatorname{Sym}^{d} S_{i}\right)\right) & =H^{a}\left(q_{i}^{*} \wedge^{j}\left(\operatorname{Sym}^{d} S_{i}\right)\right), \\
H^{a}\left(\wedge^{j}\left(\operatorname{Sym}^{d} S_{i+1}\right)\right) & =H^{a}\left(p_{i}^{*} \wedge^{j}\left(\operatorname{Sym}^{d} S_{i+1}\right)\right), \\
a & \geqslant 0
\end{aligned}
$$

из (3.15) и (3.13) выводим равенства

$$
H^{a}\left(\wedge^{j}\left(\operatorname{Sym}^{d} S_{i+1}\right)\right)=H^{a}\left(\wedge^{j}\left(\operatorname{Sym}^{d} S_{i}\right)\right), \quad 1 \leqslant i \leqslant k .
$$

Более того, полагая $j_{i}:=\operatorname{rkSym}^{d} S_{i}$, получаем

$$
\wedge^{j_{i}}\left(\operatorname{Sym}^{d} S_{i}\right) \simeq \mathscr{O}_{G(i, V)}\left(-\left(\begin{array}{c}
d+i-1 \\
i
\end{array}\right)\right),
$$

так что по аналогии с (3.15)

$$
H^{a}\left(\wedge^{j_{i}}\left(\operatorname{Sym}^{d} S_{i}\right)\right)=0, \quad a \geqslant 0, \quad 1 \leqslant i \leqslant k .
$$

Эти равенства вместе с (3.16) дают (3.8).

Равенства $(3.7)$ и $(3.8)$ показывают, что $h^{0}\left(\mathscr{O}_{B_{H}}\right)=h^{0}\left(\mathscr{O}_{G(k+1, V)}\right)=1$. Следовательно, $B_{H}$ связно. Это вместе с гладкостью $B_{H}$ влечет его неприводимость.

Рассмотрим график инцидентности $\Sigma_{H}=\left\{\left(x, \mathbb{P}^{k}\right) \in H \times B_{H} \mid x \in \mathbb{P}^{k}\right\}$ с проекциями

$$
H \stackrel{\pi_{1}}{\leftarrow} \Sigma_{H} \stackrel{\pi_{2}}{\rightarrow} B_{H}
$$

Поскольку слои проекции $\pi_{2}$ изоморфны $\mathbb{P}^{k}$, неприводимость $B_{H}$ влечет неприводимость $\Sigma_{H}$. 
Лемма 2. Пусть $H \subset \mathbb{P}^{n}$ - гладкая гиперповерхность, общая в смысле (3.4). Тогда:

(i) ного $x \in H$ множество $B_{H}(x):=\pi_{2}\left(\pi_{1}^{-1}(x)\right)$ равноразмерно размерности

$$
\operatorname{dim} B_{H}(x)=k(n-k)-\left(\begin{array}{c}
k+d \\
d
\end{array}\right)+1 ;
$$

более того, для общего $x \in H B_{H}(x)$ является неприводимым подмногообразием в $B_{H}$;

(ii) подмножество $K_{H, k}(x):=\pi_{1}\left(\pi_{2}^{-1}\left(B_{H}(x)\right)\right)=\bigcup_{\mathbb{P}^{k} \in B_{H}(x)} \mathbb{P}^{k}$ в $H$ uмеет размерность

$$
\operatorname{dim} K_{H, k}(x) \geqslant n-d ;
$$

более того, для общего $x \in H K_{H, k}(x)$ есть неприводимое подмногообразие в $H$.

Доказательство. (i) Пусть $\left(x, \mathbb{P}^{k}\right) \in \Sigma_{H}$. Рассмотрим стандартную резольвенту Кошуля пучка идеалов $\mathscr{I}_{x, \mathbb{P}^{k}}$

$$
0 \rightarrow \mathscr{O}_{\mathbb{P} k}(-k) \rightarrow \cdots \rightarrow \mathscr{O}_{\mathbb{P} k}(-i)^{\left(\begin{array}{c}
k \\
i
\end{array}\right)} \rightarrow \cdots \rightarrow \mathscr{O}_{\mathbb{P} k}(-1)^{k} \rightarrow \mathscr{I}_{x, \mathbb{P}^{k}} \rightarrow 0 .
$$

Подкручивая (3.20) на $N_{\mathbb{P} k} / H$, получим точную последовательность

$$
\begin{aligned}
0 & \rightarrow N_{\mathbb{P} k / H}(-k) \rightarrow \cdots \rightarrow N_{\mathbb{P} k / H}(-i)^{\left(\begin{array}{c}
k \\
i
\end{array}\right)} \rightarrow \cdots \rightarrow N_{\mathbb{P} k / H}(-1)^{k} \\
& \rightarrow \mathscr{I}_{x, \mathbb{P} k} \otimes N_{\mathbb{P}^{k} / H} \rightarrow 0 .
\end{aligned}
$$

Поскольку $h^{i}\left(N_{\mathbb{P}^{k} / H}(-i)\right)=0,1 \leqslant i \leqslant k$ (для $i>1$ эти равенства немедленно следуют из (3.3); для $i=1$ см. (3.5)), то (3.21) дает

$$
h^{1}\left(\mathscr{I}_{x, \mathbb{P}^{k}} \otimes N_{\mathbb{P}^{k} / H}\right)=0 .
$$

Далее, рассмотрим точную тройку

$$
0 \rightarrow \mathscr{I}_{x, \mathbb{P}^{k}} \otimes N_{\mathbb{P}^{k} / H} \rightarrow N_{\mathbb{P}^{k} / H} \rightarrow \mathbb{C}_{x} \otimes N_{\mathbb{P}^{k} / H} \rightarrow 0
$$

Так как $\mathbb{C}_{x} \otimes N_{\mathbb{P k} / H} \simeq \mathbb{C}^{n-1-k}$, из $(3.5),(3.23)$ и (3.22) следует, что

$$
h^{0}\left(\mathscr{I}_{x, \mathbb{P}^{k}} \otimes N_{\mathbb{P}^{k} / H}\right)=k(n-k)-\left(\begin{array}{c}
k+d \\
d
\end{array}\right)+1 .
$$

Заметим, что $H^{0}\left(\mathscr{I}_{x, \mathbb{P} k} \otimes N_{\mathbb{P}^{k} / H}\right)$ есть касательное пространство по Зарискому к $B_{H}(x)$ в точке $\mathbb{P}^{k}$. Более того, из (3.22) и (3.24) по теории деформации вытекает гладкость $B_{H}(x)$ в точке $\mathbb{P}^{k}$ и равноразмерность $B_{H}(x)$ вместе с равенством (3.18). Это последнее равенство показывает, что $\operatorname{dim} \pi_{1}\left(\Sigma_{H}\right)=\operatorname{dim} H$. Поскольку $H$ неприводимо, морфизм $\pi_{1}: \Sigma_{H} \rightarrow H$ является сюръективным, будучи проективным морфизмом проективных многообразий. Это означает, что $H$ заметается пространствами $\mathbb{P}^{k} \in B_{H}$. 
(ii) Пусть теперь $y$ - произвольная точка в $\mathbb{P}^{k}$, отличная от $x$, и пусть $\mathbb{P}^{1}-$ проективная прямая в $\mathbb{P}^{k}$, соединяющая точки $x$ и $y$. Подкручивая (3.3) на пучки $\mathscr{I}_{\mathbb{P}^{1}, \mathbb{P}^{k}}$ и $\mathscr{O}_{\mathbb{P}^{1}}(-2)$, получаем точные тройки

$$
\begin{gathered}
0 \rightarrow \mathscr{I}_{\mathbb{P}^{1}, \mathbb{P}^{k}} \otimes N_{\mathbb{P}^{k} / H} \rightarrow \mathscr{I}_{\mathbb{P}^{1}, \mathbb{P}^{k}} \otimes \mathscr{O}_{\mathbb{P}^{k}}(1)^{n-k} \stackrel{\varepsilon_{k}}{\rightarrow} \mathscr{I}_{\mathbb{P}^{1}, \mathbb{P}^{k}} \otimes \mathscr{O}_{\mathbb{P}^{k}}(d) \rightarrow 0 \\
0 \rightarrow N_{\mathbb{P}^{k} / H} \otimes \mathscr{O}_{\mathbb{P}^{1}}(-2) \rightarrow \mathscr{O}_{\mathbb{P}^{1}}(-1)^{n-k} \rightarrow \mathscr{O}_{\mathbb{P}^{1}}(d-2) \rightarrow 0
\end{gathered}
$$

Рассмотрим морфизм $\varepsilon_{k}$ в (3.25). Переходя к сечениям, получаем гомоморфизм

$$
H^{0}\left(\varepsilon_{k}\right): H^{0}\left(\mathscr{I}_{\mathbb{P}^{1}, \mathbb{P}^{k}} \otimes \mathscr{O}_{\mathbb{P}^{k}}(1)^{n-k}\right) \rightarrow H^{0}\left(\mathscr{I}_{\mathbb{P}^{1}, \mathbb{P}^{k}} \otimes \mathscr{O}_{\mathbb{P}^{k}}(d)\right) .
$$

Чтобы показать, что $H^{0}\left(\varepsilon_{k}\right)$ является эпиморфизмом, рассмотрим стандартную резольвенту Кошуля пучка $\mathscr{I}_{\mathbb{P}^{1}, \mathbb{P} k} \otimes \mathscr{O}_{\mathbb{P} k}(1)$

$$
0 \rightarrow \mathscr{O}_{\mathbb{P}^{k}}(2-k) \rightarrow \cdots \rightarrow \mathscr{O}_{\mathbb{P}^{k}}(-1)^{\left(\begin{array}{c}
k-1 \\
2
\end{array}\right)} \rightarrow \mathscr{O}_{\mathbb{P}^{k}}^{k-1} \stackrel{e_{1}}{\rightarrow} \mathscr{I}_{\mathbb{P}^{1}, \mathbb{P}^{k}} \otimes \mathscr{O}_{\mathbb{P}^{k}}(1) \rightarrow 0
$$

Переходя к когомологиям, получаем эпиморфизм в сечениях

$$
H^{0}\left(e_{1}\right): H^{0}\left(\mathscr{O}_{\mathbb{P}^{k}}^{k-1}\right) \rightarrow H^{0}\left(\mathscr{I}_{\mathbb{P}^{1}, \mathbb{P}^{k}} \otimes \mathscr{O}_{\mathbb{P}^{k}}(1)\right) .
$$

Подкручивая предыдущую резольвенту на $\mathscr{O}_{\mathbb{P} k}(d-1)$ и снова переходя к когомологиям, получаем эпиморфизм

$$
H^{0}\left(e_{d}\right): H^{0}\left(\mathscr{O}_{\mathbb{P}^{k}}(d-1)^{k-1}\right) \rightarrow H^{0}\left(\mathscr{I}_{\mathbb{P}^{1}, \mathbb{P}^{k}} \otimes \mathscr{O}_{\mathbb{P}^{k}}(d)\right) .
$$

Гомоморфизмы $H^{0}\left(\varepsilon_{k}\right), H^{0}\left(e_{1}\right)$ и $H^{0}\left(e_{d}\right)$ образуют коммутативную диаграмму

$$
\begin{gathered}
H^{0}\left(\mathscr{I}_{\mathbb{P}^{1}, \mathbb{P}^{k}} \otimes \mathscr{O}_{\mathbb{P}^{k}}(1)^{n-k}\right)^{H^{0}\left(\varepsilon_{k}\right)} \longrightarrow H^{0}\left(\mathscr{I}_{\mathbb{P}^{1}, \mathbb{P}^{k}} \otimes \mathscr{O}_{\mathbb{P}^{k}}(d)\right) \\
H^{0}\left(e_{1}\right)^{n-k} \uparrow \\
H^{0}\left(\mathscr{O}_{\mathbb{P}^{k}}^{(k-1)(n-k)}\right) \stackrel{H^{0}\left(\varepsilon_{k}\right)^{k-1}}{\longrightarrow} H^{0}\left(\mathscr{O}_{\mathbb{P}^{k}}(d-1)^{k-1}\right)
\end{gathered}
$$

в которой сюръективность нижнего горизонтального отображения $H^{0}\left(\varepsilon_{k}\right)^{k-1}$ следует из (3.4). Следовательно, $H^{0}\left(\varepsilon_{k}\right)$ - эпиморфизм. Поэтому когомологическая последовательность тройки (3.25) дает

$$
\begin{gathered}
h^{0}\left(\mathscr{I}_{\mathbb{P}^{1}, \mathbb{P}^{k}} \otimes N_{\mathbb{P}^{k} / H}\right)=(k-1)(n-k)-\left(\begin{array}{c}
k+d \\
d
\end{array}\right)+d+1, \\
h^{1}\left(\mathscr{I}_{\mathbb{P}^{1}, \mathbb{P}^{k}} \otimes N_{\mathbb{P}^{k} / H}\right)=0 .
\end{gathered}
$$

Далее, (3.26) влечет $h^{0}\left(N_{\mathbb{P}^{k} / H} \otimes \mathscr{O}_{\mathbb{P}^{1}}(-2)\right)=0, h^{1}\left(N_{\mathbb{P}^{k}} / H \otimes \mathscr{O}_{\mathbb{P}^{1}}(-2)\right)=d-1$. Отсюда и из (3.27) и точной тройки

$$
0 \rightarrow \mathscr{I}_{\mathbb{P}^{1}, \mathbb{P}^{k}} \otimes N_{\mathbb{P}^{k} / H} \rightarrow \mathscr{I}_{x \cup y, \mathbb{P}^{k}} \otimes N_{\mathbb{P}^{k} / H} \rightarrow \mathscr{O}_{\mathbb{P}^{1}}(-1) \otimes N_{\mathbb{P}^{k} / H} \rightarrow 0
$$

находим

$$
\begin{gathered}
h^{0}\left(\mathscr{I}_{x \cup y, \mathbb{P}^{k}} \otimes N_{\mathbb{P}^{k} / H}\right)=(k-1)(n-k)-\left(\begin{array}{c}
k+d \\
d
\end{array}\right)+d+1, \\
h^{1}\left(\mathscr{I}_{x \cup y, \mathbb{P}^{k}} \otimes N_{\mathbb{P}^{k} / H}\right)=d-1 .
\end{gathered}
$$


Положим $\Sigma_{H}(x):=\pi_{2}^{-1}\left(B_{H}(x)\right), \pi_{1}(x):=\left.\pi_{1}\right|_{\Sigma_{H}(x)}$, и пусть

$$
K_{H, k}(x) \stackrel{\pi_{1}(x)}{\longleftarrow} \Sigma_{H}(x) \stackrel{\pi_{2}(x)}{\longrightarrow} B_{H}(x)
$$

- диаграмма проекций. Для любой точки $y \in K_{H, k}(x), y \neq x$, рассмотрим слой $B_{H, x}(y):=\pi_{1}(x)^{-1}(y)$ как подмногообразие в $B_{H}(x)$. Касательное пространство по Зарискому к $B_{H, x}(y)$ в точке в $\mathbb{P}^{k}$ совпадает с $H^{0}\left(\mathscr{I}_{x \cup y, \mathbb{P}^{k}} \otimes N_{\mathbb{P}^{k} / H}\right)$, поэтому в силу (3.29) и теории деформаций имеем

$$
(k-1)(n-k)-\left(\begin{array}{c}
k+d \\
d
\end{array}\right)+d+1 \geqslant \operatorname{dim} B_{H, x}(y) \geqslant(k-1)(n-k)-\left(\begin{array}{c}
k+d \\
d
\end{array}\right)+2 .
$$

Ясно, что $\operatorname{dim} B_{H, x}(y)>0$, а значит, $\pi_{1}(x)$ сюръективно. Поскольку слой проекции $\pi_{2}(x)$ есть $\mathbb{P}^{k}$, это в совокупности с $(3.18)$, (3.30) и неприводимостью $B_{H}(x)$ влечет неравенство (3.19) и неприводимость $K_{H, k}(x)$.

Как следствие этой леммы мы получаем следующую теорему.

Теорема 2. Всякая гиперповерхность H степени d в $\mathbb{P}^{n}$ заметается подпространствами $\mathbb{P}^{k} \subset \mathbb{P}^{n}$.

ДокАЗАтЕЛЬСтво. Рассмотрим графики инцидентности $\Pi:=\left\{\left(\mathbb{P}^{k}, x\right) \in\right.$ $\left.G(k+1, V) \times \mathbb{P}^{n} \mid x \in \mathbb{P}^{k}\right\}$ и $\widetilde{H}:=\left\{(H, x) \in \mathbb{P}^{s} \times \mathbb{P}^{n} \mid x \in H\right\}$, входящие в коммутативную диаграмму

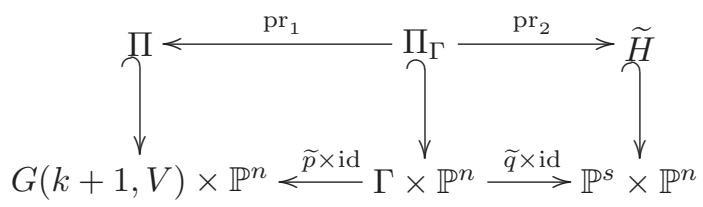

где $\Gamma, \widetilde{p}$ и $\widetilde{q}$ были определены в $(3.2), \Pi_{\Gamma}=(\widetilde{p} \times \mathrm{id})^{-1}(\Pi), \mathrm{a} \mathrm{pr}_{1}$ и $\mathrm{pr}_{2}-$ индуцированные проекции. Поскольку общая гладкая гиперповерхность $H \in \mathbb{P}^{s}$ заметается проективными подпространствами $\mathbb{P}^{k} \subset \mathbb{P}^{n}$ (лемма 2, (i)), проекция $\mathrm{pr}_{2}$ доминантна. Следовательно, $\mathrm{pr}_{2}$ сюръективна, поскольку все многообразия и морфизмы в (3.31) проектины. Отсюда следует утверждение теоремы.

\section{3. Проективные подпространства в многообразиях ограниченной} коразмерности и степени и растущей размерности. Пусть $X \subset \mathbb{P}^{n}$ есть неприводимое проективное многообразие, удовлетворяющее условиям

$$
1 \leqslant c:=\operatorname{codim}_{\mathbb{P} n} X, \quad \operatorname{deg} X \leqslant d,
$$

где $c$ - константа. Предположим, что $\mathbb{P}^{n}=\operatorname{Span} X$. Хорошо известно, что $\operatorname{deg} X \geqslant c+1$. Если $c \geqslant 2$, то, начиная с $X_{0}:=X$, можно построить индуктивно последовательность проективных многообразий $X_{i} \subset \mathbb{P}^{n-i}, 0 \leqslant i \leqslant c-1$, соответствующих коразмерностей $c-i$ вместе с линейными проекциями

$$
p_{x_{i}}: \mathbb{P}^{n-i} \rightarrow \mathbb{P}^{n-i-1}, \quad 0 \leqslant i \leqslant c-2,
$$

с центрами в точках $x_{i} \in X_{i} \backslash \operatorname{Sing} X_{i}$ таких, что каждое ограничение

$$
p_{i}:=\left.p_{x_{i}}\right|_{X_{i}}: X_{i} \rightarrow X_{i+1}, \quad 0 \leqslant i \leqslant c-2,
$$


является бирациональным изоморфизмом. Для этого достаточно зафиксировать точку $x_{i} \in X_{i} \backslash \operatorname{Sing} X_{i}$ и взять в качестве $X_{i+1}$ замыкание многообразия $p_{x_{i}}\left(X_{i}\right)$ в $\mathbb{P}^{n-i}$. Тогда $\operatorname{deg} X_{i+1}=\operatorname{deg} X_{i}-1$. Утверждение о том, что $p_{i}$ бирационально, является стандартным.

Далее, используя обозначение (3.1), положим

$$
\begin{gathered}
k_{c-1}(n):=k(n-c+1), \quad k_{c-1-i}(n):=\underbrace{\left[\frac{1}{2} \cdots\left[\frac{1}{2}\left[\frac{1}{2} k_{c-1}(n)\right]\right] \cdots\right]}_{i}, \\
1 \leqslant i \leqslant c-1 .
\end{gathered}
$$

Теперь обратной индукцией покажем, что $X=X_{0}$ заметается проективными подпространствами размерности $k_{0}(n)$. По определению $X_{c-1}$ есть гиперповерхность в $\mathbb{P}^{n-(c-1)}$ степени

$$
\operatorname{deg} X_{c-1}=\operatorname{deg} X-(c-1) \leqslant d .
$$

Следовательно, по теореме $2 X_{c-1}$ заметается подпространствами $\mathbb{P}^{k_{c-1}(n)}$ в $\mathbb{P}^{n-(c-1)}$. Это дает начало индукции.

Переходя к шагу индукции, рассмотрим бирациональное отображение (3.33). Предположим, что $X_{i+1}$ заметается подпространствами $\mathbb{P}^{k_{i+1}(n)} \subset \mathbb{P}^{n-i-1}$. Пусть $B$ - неприводимая компонента базы семейства всех таких подпространств, удовлетворяющая условию, что подпространства из $B$ заметают $X_{i+1}$. Возьмем общее пространство $\mathbb{P}^{k_{i+1}(n)} \in B$ и рассмотрим замыкание $Y_{i+1}:=\overline{p_{i}^{-1}\left(\mathbb{P}^{k_{i+1}(n)}\right)}$. Так как $\mathbb{P}^{k_{i+1}}$ - общая точка из $B$, то рациональное отображение

$$
\widetilde{p}:=\left.p_{i}\right|_{Y_{i+1}}: Y_{i+1} \rightarrow \mathbb{P}^{k_{i+1}(n)}
$$

есть линейная проекция из точки $x_{i} \in Y_{i+1}$, и имеет место один из следующих случаев:

(i) $Y_{i+1}$ - неприводимая квадрика и

$$
\widetilde{p}: Y_{i+1} \rightarrow \mathbb{P}^{k_{i+1}(n)}
$$

есть бирациональная (стереографическая) проекция из точки $x_{i} \in Y_{i+1}$;

(ii) $Y_{i+1}$ - приводимая квадрика, содержащая в качестве компоненты некотоpoе $k_{i+1}(n)$-мерное пространство $\widetilde{\mathbb{P}}^{k_{i+1}(n)}$, отображаемое изоморфно на $\mathbb{P}^{k_{i+1}(n)}$,

$$
\widetilde{p}: \widetilde{\mathbb{P}}^{k_{i+1}(n)} \stackrel{\sim}{\rightarrow} \mathbb{P}^{k_{i+1}(n)} .
$$

Рассмотрим оба эти случая.

В случае (i) $Y_{i+1}$ есть неприводимая квадрика размерности $k_{i+1}(n)$, заполняемая проективными подпространствами размерности $\left[\frac{1}{2} k_{i+1}(n)\right]=k_{i}(n)$. Поскольку $\mathbb{P}^{k_{i+1}(n)}$ есть общая точка в $B$, квадрики $Y_{i+1}\left(\mathbb{P}^{k_{i+1}(n)}\right), \mathbb{P}^{k_{i+1}(n)} \in B$, заметают многообразие $X_{i}$. Следовательно, подпространства $\mathbb{P}^{k_{i}(n)}$ заметают $X_{i}$.

В случае (ii) из неприводимости $X_{i}$ и бирациональности $p_{i}$ следует, что подпространства $\widetilde{\mathbb{P}}^{k_{i+1}(n)}$ заметают $X_{i}$. Более того, каждое $\widetilde{\mathbb{P}}^{k_{i+1}(n)}$ заметается подпространствами $\mathbb{P}^{k_{i}(n)}$. Следовательно, $X_{i}$ также заметается этими подпространствами $\mathbb{P}^{k_{i}(n)}$.

Наконец, заметим, что $\lim _{n \rightarrow \infty} k_{0}(n)=\infty$. Таким образом, доказана следующая теорема. 
Теорема 3. Пусть $X \subset \mathbb{P}^{n}$ - неприводимое проективное многообразие, удовлетворяющее условиям (3.32), u $\operatorname{Span} X=\mathbb{P}^{n}$. Тогда $X$ заметается проективными подпространствами $\mathbb{P}^{k_{0}(n)} \subset \mathbb{P}^{n} c \lim _{n \rightarrow \infty} k_{0}(n)=\infty$.

3.4. Цепочки проективных подпространств, соединяющих точки многообразий ограниченной коразмерности и степени. Пусть снова $H-$ гладкая гиперповерхность степени $d \geqslant 2$ в $\mathbb{P}^{n}$ и $x \in H$. Обозначим через $\mathbb{P}^{n-1}(x)$ гиперплоскость в $\mathbb{P}^{n}$, касательную к $H$ в точке $x$. Возьмем аффинное подпространство $\mathbb{A}^{n-1}(x)$ в $\mathbb{P}^{n-1}(x)$, содержащее точку $x$, с аффинными координатами $\left(y_{1}, \ldots, y_{n-1}\right)$ в окрестности $x$. Пересечение $Y_{H}(x):=H \cap \mathbb{A}^{n-1}(x)$ есть гиперповерхность в $\mathbb{A}^{n-1}(x)$, задаваемая уравнением $\Psi_{x}=0$ для некоторого многочлена $\Psi_{x}=\Psi_{x}\left(y_{1}, \ldots, y_{n-1}\right)$ степени $d$. Разложим $\Psi_{x}$ в сумму его однородных компонент:

$$
\Psi_{x}=\sum_{p=2}^{d} \Psi_{p}\left(y_{1}, \ldots, y_{n-1}\right), \quad \operatorname{deg} \Psi_{p}=p .
$$

Рассмотрим $\left(y_{1}: y_{2}: \ldots: y_{n-1}\right)$ как однородные координаты в $\mathbb{P}^{n-2}$; соответственно, рассмотрим $\Psi_{p}$ как формы $\Psi_{p} \in H^{0}\left(\mathscr{O}_{\mathbb{P}^{n-2}}(p)\right)$. Имеем замкнутое подмножество

$$
X_{x}=\bigcap_{p=2}^{d}\left\{\Psi_{p}\left(y_{1}, \ldots, y_{n-1}\right)=0\right\}, \quad \operatorname{deg} \Psi_{p}=p,
$$

в $\mathbb{P}^{n-2}$. Теорема Безу дает

$$
\operatorname{codim}_{\mathbb{P}^{n-2}} X \leqslant d-1, \quad \operatorname{deg} X \leqslant d !
$$

для любой неприводимой компоненты $X$ в $X_{x}$. Поэтому $n-2 \geqslant \operatorname{dim} \operatorname{Span} X \geqslant$ $n-d-1$. В частности, коразмерность и степень $X$ ограничены не зависящими от $n$ константами, так что теорема 3 применима к $X$. Это доказывает следующую лемму.

Лемма 3. Существует число $n(d) \in \mathbb{Z}_{>0}$ такое, что при $n \geqslant n(d)$ многообразие $X_{x}$ связно, и любая неприводимая компонента $X$ многообразия $X_{x}$ заметается подпространствами $\mathbb{P}^{\widetilde{k}(n)} \subset \mathbb{P}^{n-2} c \lim _{n \rightarrow \infty} \widetilde{k}(n)=\infty$.

Пусть $K_{H}(x)$ - конус в $\mathbb{A}^{n-1}(x)$ с базой $X_{x}$. По лемме 3 замыкание $\overline{K_{H}(x)}$ многообразия $K_{H}(x)$ в $\mathbb{P}^{n-1}(x)$ заметается подпространствами $\mathbb{P}^{\widetilde{k}(n)}$. Рассмотрим подмногообразие $K_{H, k}(x)$ в $H$, определенное в лемме 2 , (ii). По определению $K_{H, k}(x)$ заметается подпространствами $\mathbb{P}^{k}$ на $H$, проходящими через точку $x$. Ясно, что

$$
K_{H}(x) \supset K_{H, k}(x), \quad k=\widetilde{k}(n) .
$$

Предположим, что $H \subset \mathbb{P}^{n}$ - общая гладкая гиперповерхность степени $d$ и $x$ - общая точка в $H$. В частности, формы $\Psi_{p}$ являются общими точками пространств $H^{0}\left(\mathscr{O}_{\mathbb{P}^{n-2}}(p)\right)$. Следовательно, в этом случае $X=X_{x}$ есть гладкое неприводимое полное пересечение $d-1$ гиперповерхностей $\left\{\Psi_{p}=0\right\}$, $p=2, \ldots, d$, в $\mathbb{P}^{n-2}$, и неравенства (3.36) становятся равенствами. Это вместе 
с $(3.37),(3.19)$ влечет равенство $K_{H}(x)=K_{H, k}(x)$. В частности, пучок $\mathscr{O}_{X}$ имеет стандартную резольвенту Кошуля

$$
0 \rightarrow \mathscr{O}_{\mathbb{P}^{n-1}}\left(2-\frac{d(d+1)}{2}\right) \rightarrow \cdots \rightarrow \bigoplus_{p=2}^{d} \mathscr{O}_{\mathbb{P}^{n-2}}(1-p) \rightarrow \mathscr{O}_{\mathbb{P}^{n-2}}(1) \stackrel{\text { res }}{\rightarrow} \mathscr{O}_{X}(1) \rightarrow 0
$$

Эта резольвента вместе с (3.1) показывает, что отображение ограничения

$$
H^{0}(\text { res }): H^{0}\left(\mathscr{O}_{\mathbb{P}^{n-2}}(1)\right) \rightarrow H^{0}\left(\mathscr{O}_{X}(1)\right)
$$

является изоморфизмом. Поэтому $\operatorname{Span} X=\mathbb{P}^{n-2}$ и, следовательно,

$$
\operatorname{Span} K_{H, k}(x)=\mathbb{P}^{n-1}(x) .
$$

Определим теперь последовательность неприводимых подмногообразий $x \in$ $X_{1} \subset X_{2} \subset \cdots \subset X_{i} \subset \cdots \subset H$ по индукции:

1) $X_{1}:=K_{H, k}(x)$;

2) $X_{i+1}:=\pi_{1}\left(\pi_{2}^{-1}\left(Y_{i}\right)\right)$ для $i \geqslant 1$, где $Y_{i}$ есть неприводимая компонента в $\pi_{2}\left(\pi_{1}^{-1}\left(X_{i}\right)\right)$, а проекции $\pi_{1}$ и $\pi_{2}$ введены в диаграмме $(3.17)$.

Поскольку $X$ неприводимо, эта последовательность стабилизируется, т.е.

$$
X_{1} \subset X_{2} \subset \cdots \subset X_{i_{0}}=X_{i_{0}+1} \subset \cdots \subset H
$$

для некоторого номера $i_{0}$. Рассмотрим плотное открытое подмножество $U:=$ $\left\{x^{\prime} \in H \mid K_{H, k}\left(x^{\prime}\right)\right.$ неприводимо и $\left.\operatorname{Span}\left(K_{H, k}\left(x^{\prime}\right)\right)=\mathbb{P}^{n-1}\left(x^{\prime}\right)\right\} \subset H$. По построению $x \in U$, а следовательно, $X_{i_{0}} \cap U$ есть плотное открытое множество в $X_{i_{0}}$. Более того, по определению многообразия $X_{i_{0}}$ мы имеем

$$
K_{H, k}\left(x^{\prime}\right) \subset X_{i_{0}}
$$

для $x^{\prime} \in X_{i_{0}} \cap U$. Обозначим через $H\left(x^{\prime}\right)$ проективное подпространство в $\mathbb{P}^{n}$, касательное к $X_{i_{0}}$ в точке $x^{\prime} \in\left(X_{i_{0}} \backslash \operatorname{Sing} X_{i_{0}}\right) \cap U$. Поскольку $K_{H, k}\left(x^{\prime}\right)$ по определению заметается проективными подпространствами на $H$, проходящими через точку $x^{\prime}$, то из (3.40) вытекает, что $K_{H, k}\left(x^{\prime}\right) \subset H\left(x^{\prime}\right) \subset \mathbb{P}^{n-1}\left(x^{\prime}\right)$. $\mathrm{C}$ другой стороны, так как $x^{\prime} \in U$, то, значит, $\operatorname{Span} K_{H, k}(x)=H\left(x^{\prime}\right)$. Поскольку $H\left(x^{\prime}\right)$ есть подпространство в $\mathbb{P}^{n-1}\left(x^{\prime}\right)$, то в силу (3.38) имеем равенство $H\left(x^{\prime}\right)=\mathbb{P}^{n-1}\left(x^{\prime}\right)$. Следовательно, так как $x^{\prime}$ - неособая точка в $X_{i_{0}}$, получаем $\operatorname{dim} X_{i_{0}}=\operatorname{dim} H$, так что

$$
X_{i_{0}}=H
$$

Это равенство и конструкция цепочки (3.39) показывают, что точку $x \in H$ можно соединить с любой точкой $x^{\prime} \in H$ цепочкой подпространств $\mathbb{P}_{1}^{k}, \mathbb{P}_{2}^{k}, \ldots, \mathbb{P}_{i_{0}}^{k}$. Таким образом, мы имеем

$$
x \in \mathbb{P}_{1}^{k} \subset \mathbb{P}_{1}^{k} \cup \mathbb{P}_{2}^{k} \cup \cdots \cup \mathbb{P}_{i_{0}}^{k} \supset \mathbb{P}_{i_{0}}^{k} \ni x^{\prime} .
$$

Покажем, наконец, что (3.42) выполняется без предположения об общности $H$ и $x$. Это делается по-существу так же, как и в доказательстве теоремы 2. Действительно, рассмотрим грассманиан $G:=G(k+1, V)$, многообразие инцидентности

$\operatorname{Inc}^{i_{0}}(G):=\left\{\left(\mathbb{P}_{1}^{k}, \ldots, \mathbb{P}_{i_{0}}^{k}\right) \in G^{\times i_{0}} \mid \mathbb{P}_{1}^{k}, \ldots, \mathbb{P}_{i_{0}}^{k}\right.$ есть цепочка подпространств в $\left.\mathbb{P}^{n}\right\}$ 
и графики инцидентности

$$
\begin{gathered}
\Pi_{i_{0}}:=\left\{\left(\mathbb{P}_{1}^{k}, \ldots, \mathbb{P}_{i_{0}}^{k}, x, x^{\prime}\right) \in \operatorname{Inc}^{i_{0}}(G) \times \mathbb{P}^{n} \times \mathbb{P}^{n} \mid x \in \mathbb{P}_{1}^{k}, x^{\prime} \in \mathbb{P}_{i_{0}}^{k}\right\}, \\
\widetilde{\widetilde{H}}:=\left\{\left(H, x, x^{\prime}\right) \in \mathbb{P}^{s} \times \mathbb{P}^{n} \times \mathbb{P}^{n} \mid x, x^{\prime} \in H\right\} \\
\Gamma_{i_{0}}:=\left\{\left(\mathbb{P}_{1}^{k}, \ldots, \mathbb{P}_{i_{0}}^{k}, G\right) \in \operatorname{Inc}^{i_{0}}(G) \times \mathbb{P}^{s} \mid \mathbb{P}_{1}^{k}, \ldots, \mathbb{P}_{i_{0}}^{k} \subset H\right\}
\end{gathered}
$$

с естественными проекциями

$$
\operatorname{Inc}^{i_{0}}(G) \stackrel{\widetilde{p}_{i_{0}}}{\longleftarrow} \Gamma_{i_{0}} \stackrel{\widetilde{q}_{i_{0}}}{\longrightarrow} \mathbb{P}^{s} .
$$

Имеем коммутативную диаграмму

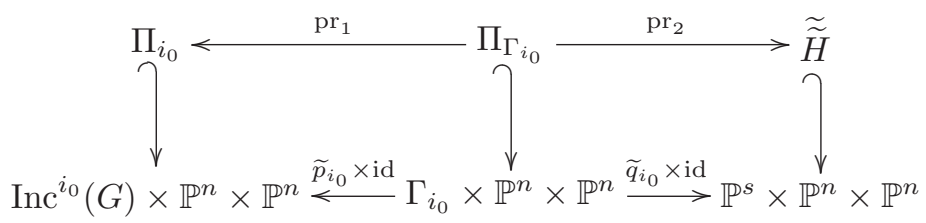

где $\mathrm{pr}_{1}$ и $\mathrm{pr}_{2}$ - индуцированные проекции. Так как общая гладкая гиперповерхность $H \in \mathbb{P}^{s}$ заметается проективными подпространствами $\mathbb{P}^{k} \subset \mathbb{P}^{n}$ (лемма 2,(i)), морфизм $\mathrm{pr}_{2}$ доминантен. Следовательно, он сюръективен, поскольку все многообразия и морфизмы в вышеприведенной диаграмме проективны. Это равносильно (3.42) для произвольной гиперповерхности $H \in \mathbb{P}^{s}$ и произвольных точек $x, x^{\prime} \in H$.

Итак, мы доказали следующую лемму.

Лемма 4. Пусть $H$ - гиперповерхность степени $d$ в $\mathbb{P}^{n}$. Тогда любие две различные точки $x, x^{\prime} \in H$ можно соединить иепочкой (3.42) подпространств $\mathbb{P}^{k}$ на $\mathrm{H}$.

Наконец, лемма 4 вместе с теоремой 3 дают основной результат этого параграфа.

Теорема 4. В условиях теоремы 3 любъе две различные точки $x, x^{\prime} \in X$ можно соединить цепочкой (3.42) подпространств $\mathbb{P}^{k_{0}(n)}$ на $X$, где

$$
\lim _{n \rightarrow \infty} k_{0}(n)=\infty
$$

\section{$\S 4$. Достаточное условие $m$-регулярности векторного расслоения на $\mathbb{P}^{N}$}

4.1. Напомним, что векторное расслоение $E$ на схеме $Y$ называется обильнъцм, если обратимый пучок Гротендика $\mathscr{O}_{\mathbb{P}\left(E^{\vee}\right)}(1)$ на $\mathbb{P}\left(E^{\vee}\right)$ обилен. Следующий результат хорошо известен (см., например, [11; предложение 6.3.56]).

Лемма 5. Пусть Е-векторное расслоение на $\mathbb{P}^{N}$. Тогда Е(а) обильно для любого $a \in \mathbb{Z}_{\geqslant a_{0}}$, где $a_{0}$ - некоторое фиксированное иелое число.

Лемма 6. Пусть $E$ - векторное расслоение на $\mathbb{P}^{1}$. Тогда $Е(a)$ обильно для $a \geqslant 1-\delta_{B}(E)$. 
ДокАЗАтЕльство. По теореме Гротендика $E \simeq \bigoplus_{j=1}^{r} \mathscr{O}_{\mathbb{P} 1}\left(a_{j}\right)$, где $\delta_{B}(E)=$ $a_{1} \leqslant a_{2} \leqslant \cdots \leqslant a_{r}, r=\mathrm{rk} E$. Следовательно, для $a \geqslant 1-\delta_{B}(E)$ расслоение $E(a)$ есть прямая сумма обильных линейных расслоений. Тогда согласно [11; предложение $6.1 .12,(\mathrm{i})] \quad E(a)$ само является обильным.

4.2. Напомним теперь понятие степени $\operatorname{deg} \mathscr{E}$ векторного расслоения $\mathscr{E}$ на одномерной схеме $Y$. Если $Y$ - гладкая неприводимая кривая, то $\operatorname{deg} \mathscr{E}:=$ $\chi(\mathscr{E})-\chi\left(\mathscr{O}_{Y}\right) \operatorname{rk} \mathscr{E}$. Если $Y$ неприводимая, но не обязательно гладкая кривая, то $\operatorname{deg} \mathscr{E}$ определяется как степень обратного образа $\mathscr{E}$ относительно нормализации $Y$. Если $Y$ есть общая одномерная схема с неприводимыми компонентами $Y_{1}, \ldots, Y_{q}$, то корректно определены кратности $k_{i} \in \mathbb{Z}_{>0}$ компонент $Y_{i}$ в $Y$ (см. [12; п. 1.5]), и мы полагаем

$$
\operatorname{deg} \mathscr{E}=\sum_{i} k_{i} \operatorname{deg}\left(\left.\mathscr{E}\right|_{Y_{i}}\right)
$$

Лемма 7. Пусть $E$ - векторное расслоение на $\mathbb{P}^{N} u$ pr: $\mathbb{P}\left(E^{\vee}\right) \rightarrow \mathbb{P}^{N}$ - проекиия. Пусть $Y$ - одномерная подсхема в $\mathbb{P}\left(E^{\vee}\right)$ такая, ито $Y_{\text {red }} \subset \operatorname{pr}^{-1}\left(\mathbb{P}^{1}\right)$ для некоторой прямой $\mathbb{P}^{1} \subset \mathbb{P}^{N}$. Рассмотрим линейное расслоение $L_{0}=$ $\mathscr{O}_{\mathbb{P}\left(E^{\vee}\right)}(1) \otimes \operatorname{pr}^{*} \mathscr{O}_{\mathbb{P} 1}(a)$ на $\mathbb{P}\left(E^{\vee}\right)$ для $a \geqslant 1-\delta_{B}(E)$. Тогда

$$
\operatorname{deg}\left(\left.L_{0}\right|_{Y}\right)>0
$$

ДокАЗАТЕЛЬСтво. Согласно (4.1)

$$
\operatorname{deg}\left(\left.L_{0}\right|_{Y}\right)=\sum_{i} k_{i} \operatorname{deg}\left(\left.L_{0}\right|_{Y_{i}}\right), \quad k_{i}>0,
$$

где $Y_{i}$ - неприводимые компоненты в $Y$. Так как $\delta_{B}\left(\left.E\right|_{\mathbb{P}^{1}}\right) \geqslant \delta_{B}(E)$, из леммы 6 следует, что пучок $\left.L_{0}\right|_{\mathrm{pr}^{-1}\left(\mathbb{P}^{1}\right)}$ обилен. Значит, $\operatorname{deg}\left(\left.L_{0}\right|_{Y_{i}}\right)>0$ для каждой из компонент $Y_{i}$, и поэтому (4.2) следует из (4.3).

4.3. Пусть $Z_{1}-$ произвольная приведенная неприводимая кривая в $\mathbb{P}^{N}$, где $N \geqslant 3$. Возьмем проективную прямую $l_{0} \subset \mathbb{P}^{N}$ и подпространство $\mathbb{P}^{N-2} \subset \mathbb{P}^{N}$ такие, что

$$
l_{0} \cap Z_{1}=\mathbb{P}^{N-2} \cap Z_{1}=\varnothing .
$$

Зафиксируем однородные координаты $\left(x_{0}: \ldots: x_{N}\right)$ в $\mathbb{P}^{N}$, в которых

$$
l_{0}=\left\{x_{2}=\cdots=x_{N}=0\right\}, \quad \mathbb{P}^{N-2}=\left\{x_{0}=x_{1}=0\right\},
$$

и зафиксируем изоморфизм

$$
\Lambda: \mathbb{C}^{*} \times \mathbb{P}^{N} \stackrel{\sim}{\rightarrow} \mathbb{C}^{*} \times \mathbb{P}^{N}, \quad\left(t,\left(x_{0}: \ldots: x_{N}\right)\right) \mapsto\left(t,\left(x_{0}: x_{1}: t x_{2}: \ldots: t x_{N}\right)\right),
$$

$\mathbb{C}^{*}=\mathbb{C} \backslash\{0\}$. Положим $\Gamma^{*}:=\Lambda\left(\mathbb{C}^{*} \times Z_{1}\right)$ и рассмотрим схему Гильберта $\mathscr{H}:=\operatorname{Hilb}^{P_{Z_{1}}}\left(\mathbb{P}^{N}\right)$, где $P_{Z_{1}}$ есть многочлен Гильберта $P_{Z_{1}}(n)=\chi\left(\left.\mathscr{O}_{\mathbb{P}^{N}}(n)\right|_{Z_{1}}\right)$. По построению $\Gamma^{*} \rightarrow \mathbb{C}^{*}$ есть плоское семейство кривых над $\mathbb{C}^{*}$, а значит, оно определяет морфизм $g: \mathbb{C}^{*} \rightarrow \mathscr{H}$ такой, что $\Gamma^{*}=\Gamma \mathscr{H} \times \mathscr{H} \mathbb{C}^{*}$, где $\Gamma \mathscr{H} \subset$ $\mathbb{P}^{N} \times \mathscr{H}$ - универсальное семейство кривых. Координата $t$ отождествляет $\mathbb{C}^{*}$ с $\mathbb{P}^{1} \backslash\left\{z_{0}, z_{\infty}\right\}$, где $z_{0}=\{t=0\}, z_{\infty}=\{t=\infty\}$, и поскольку схема Гильберта $\mathscr{H}$ 
проективна, морфизм $g$ продолжается до морфизма $\widetilde{g}: \mathbb{P}^{1} \rightarrow H$. Таким образом, мы получаем плоское семейство $\varphi: \Gamma=\Gamma \mathscr{H} \times \mathscr{H} \mathbb{P}^{1} \rightarrow \mathbb{P}^{1}$ кривых над $\mathbb{P}^{1}$ такое, что $Z_{1}=\varphi^{-1}\left(z_{1}\right)$ для $z_{1}:=\{t=1\}$ и $\left(\varphi^{-1}\left(z_{0}\right)\right)_{\text {red }}=l_{0}$.

Пусть снова $E$ - векторное расслоение ранга $\mathrm{rk} E \geqslant 2$ на $\mathbb{P}^{N}$, и пусть pr: $\mathbb{P}\left(E^{\vee}\right) \rightarrow \mathbb{P}^{N}-$ проекция. Рассмотрим проекцию $q: \Gamma \rightarrow \mathbb{P}^{N}$ и схему $\Gamma^{E}:=$ $\mathbb{P}\left(q^{*} E^{\vee}\right)=\mathbb{P}\left(E^{\vee}\right) \times_{\mathbb{P}^{N}} \Gamma$ с проекциями

$$
\mathbb{P}\left(E^{\vee}\right) \stackrel{q^{\prime}}{\longleftarrow} \Gamma^{E} \stackrel{\mathrm{pr}^{\prime}}{\rightarrow} \Gamma, \quad \rho=\varphi \circ \mathrm{pr}^{\prime}: \Gamma^{E} \rightarrow \mathbb{P}^{1} .
$$

Заметим, что по лемме 5 существует $a_{0} \in \mathbb{Z}$ такое, что линейное расслоение $A=$ $\mathscr{O}_{\mathbb{P}\left(E^{\vee}\right)}(1) \otimes \operatorname{pr}^{*} O_{\mathbb{P}^{N}}\left(a_{0}\right)$ обильно на $\mathbb{P}\left(E^{\vee}\right) ;$ следовательно, линейное расслоение $q^{\prime *} A$ является $\rho$-обильным на $\Gamma^{E}$.

Зафиксируем неприводимую кривую $Y_{1}$ в $\Gamma^{E}$ такую, что $\operatorname{pr}^{\prime}\left(Y_{1}\right)=Z_{1}$, и обозначим через $P_{Y_{1}}$ многочлен Гильберта $P_{Y_{1}}(n):=\chi\left(\left.q^{* *} A^{\otimes n}\right|_{Y_{1}}\right)$. Рассмотрим относительную схему Гильберта $\mathscr{H}_{\mathbb{P}^{1}}=\operatorname{Hilb}^{P_{Y_{1}}}\left(\Gamma^{E} / \mathbb{P}^{1}\right)$ вместе с естественным сюръективным проективным морфизмом $f: \mathscr{H}_{\mathbb{P} 1} \rightarrow \mathbb{P}^{1}$ и универсальным се-

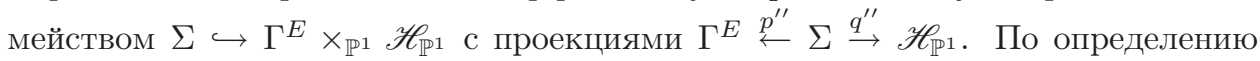
найдется точка $y_{1} \in \mathscr{H}_{\mathbb{P} 1}$ такая, что

$$
q^{\prime \prime-1}\left(y_{1}\right) \stackrel{p^{\prime \prime}}{\sim} Y_{1}
$$

и $f\left(y_{1}\right)=a_{1}$. Далее, рассмотрим нормализацию $\nu: Z \rightarrow Z_{1}$ кривой $Z_{1}$ и поверхности $\mathscr{S}=\mathbb{P}\left(\nu^{*}\left(\left.E^{\vee}\right|_{Z_{1}}\right)\right)$ и $\mathscr{S}_{1}=\mathbb{P}\left(\left.E^{\vee}\right|_{Z_{1}}\right) \subset X_{\Gamma}$ с проекциями $\operatorname{pr}_{\mathscr{S}}: \mathscr{S} \rightarrow Z$ и $\operatorname{pr}_{\mathscr{S}_{1}}: \mathscr{S}_{1} \rightarrow Z_{1}$. По конструкции морфизм $\nu$ поднимается на нормализацию $\widetilde{\nu}: \mathscr{S} \rightarrow \mathscr{S}_{1}$ так, что $\operatorname{pr}_{\mathscr{S}_{1}} \circ \widetilde{\nu}=\nu \circ \operatorname{pr}_{\mathscr{S}}$, и кривая $Y=\widetilde{\nu}^{-1}\left(Y_{1}\right)$ есть мультисечение проекции $\operatorname{pr}_{\mathscr{S}}$.

Рассмотрим многочлен Гильберта $P_{Y}(n):=\chi\left(\left.\widetilde{\nu}^{*} q^{\prime *} A^{\otimes n}\right|_{Y_{1}}\right)$. Так как $\mathscr{S}$ есть гладкая поверхность, то схема Гильберта $\operatorname{Hilb}^{P_{Y}}(\mathscr{S})$ совпадает с линейным рядом $\left|\mathscr{O}_{\mathscr{S}}(Y)\right| \simeq \mathbb{P}^{h}, h=h^{0}\left(\mathscr{O}_{\mathscr{S}}(Y)\right)-1$, и существует биективный морфизм

$$
\mathbb{P}^{h}=\operatorname{Hilb}^{P_{Y}}(\mathscr{S}) \rightarrow \operatorname{Hilb}^{P_{Y_{1}}}\left(\mathscr{S}_{1}\right)=f^{-1}\left(a_{1}\right): C \mapsto \widetilde{\nu}(C) .
$$

Таким образом, слой $f^{-1}\left(a_{1}\right)$ неприводим.

Поскольку морфизм $f: \mathscr{H}_{\mathbb{P} 1} \rightarrow \mathbb{P}^{1}$ проективен, схема $\mathscr{H}_{\mathbb{P} 1}$ также проективна. Поэтому в силу сюръективности и плоскости морфизма $f$ и неприводимости слоя $f^{-1}\left(a_{1}\right)$ существуют гладкая неприводимая кривая $T$ и морфизм $\theta: T \rightarrow \mathscr{H}_{\mathbb{P}^{1}}$ такие, что $\theta_{T}=f \circ \theta: T \rightarrow \mathbb{P}^{1}$ сюръективно. Следовательно,

$$
\theta_{T}\left(t_{0}\right)=z_{0}
$$

для некоторого $t_{0} \in T$, и поскольку $f\left(y_{1}\right)=z_{1}$, то

$$
\theta\left(t_{1}\right)=y_{1}, \quad \theta_{T}\left(t_{1}\right)=z_{1}
$$

для некоторого $t_{1} \in T$.

Рассмотрим расслоенное произведение $\Sigma_{T}=\Sigma \times_{\mathbb{P}^{1}} T$ с проекциями

$$
p_{T}: \Sigma_{T} \rightarrow T, \quad q_{T}: \Sigma_{T} \rightarrow \Sigma \stackrel{p^{\prime \prime}}{\rightarrow} X_{\Gamma} \stackrel{q^{\prime \prime}}{\rightarrow} \mathbb{P}\left(E^{\vee}\right)
$$


и вложением $i=\left(q_{T}, p_{T}\right): \Sigma \hookrightarrow \mathbb{P}\left(E^{\vee}\right) \times T$. Семейство $p_{T}: \Sigma_{T} \rightarrow T$ есть плоское семейство кривых в $\mathbb{P}\left(E^{\vee}\right)$ с базой $T$ такое, что слой $p_{T}^{-1}\left(t_{1}\right)$ совпадает с $Y_{1}$ и

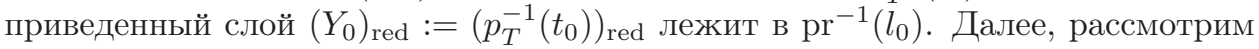
линейное расслоение $L_{T}=i^{*}\left(L_{0} \bigotimes \mathscr{O}_{T}\right)$ на $\Sigma_{T}$, где $L_{0}$ есть линейное расслоение на $X$, определенное в лемме 7. Степень $\operatorname{deg}\left(\left.L_{T}\right|_{p_{T}^{-1}(t)}\right)$ не зависит от $t \in T$ в силу принципа непрерывности (см. [12; теорема 10.2]). В частности, поскольку $\operatorname{deg}\left(\left.L_{0}\right|_{Y_{0}}\right)>0$ по лемме 7 , мы получаем

$$
\operatorname{deg}\left(\left.L_{0}\right|_{Y_{1}}\right)>0
$$

Лемма 8. Пусть $Е$ и pr те же, ито и в лемме 7.

(i) Линейное расслоение $L:=\mathscr{O}_{\mathbb{P}\left(E^{\vee}\right)}(1) \otimes \operatorname{pr}^{*} \mathscr{O}_{\mathbb{P}^{N}}\left(2-\delta_{B}(E)\right)$ на $\mathbb{P}\left(E^{\vee}\right)$ обилъно.

(ii) Линейное расслоение

$$
A_{i}:=L^{r+1} \otimes \operatorname{pr}^{*} \mathscr{O}_{\mathbb{P}^{N}}(i) \simeq \mathscr{O}_{\mathbb{P}^{\left(E^{\vee}\right)}}(r+1) \otimes \operatorname{pr}^{*} \mathscr{O}_{\mathbb{P}^{N}}\left((r+1)\left(\left(2-\delta_{B}(E)\right)+i\right)\right),
$$

где $r=\operatorname{rk} E$, такэсе обильно для произвольного $i \geqslant 0$.

ДокаЗАтельство. (i) Заметим сначала, что линейное расслоение $L_{0}:=$ $L \otimes \operatorname{pr}^{*} \mathscr{O}_{\mathbb{P}^{N}}(-1)$ численно эффективно, т.е. степень его ограничения на любую кривую в $\mathbb{P}\left(E^{\vee}\right)$ положительна. В самом деле, пусть $Y$ - неприводимая кривая в $\mathbb{P}\left(E^{\vee}\right)$. Если $\operatorname{pr}(Y)$ - кривая, то наше утверждение вытекает из (4.8). Если $\operatorname{pr}(Y)$ - точка (скажем, $z)$, то $Y \subset \operatorname{pr}^{-1}(z) \simeq \mathbb{P}^{r-1}$ и $\operatorname{deg}\left(\left.L_{0}\right|_{Y}\right)=\operatorname{deg}\left(\left.\mathscr{O}_{\mathbb{P}^{r-1}}(1)\right|_{Y}\right)$ снова положительно.

Численно эффективный класс дивизоров $c_{1}\left(L_{0}\right)$ равен $W+\left(1-\delta_{B}(E)\right) H$, где $W:=c_{1}\left(\mathscr{O}_{\mathbb{P}\left(E^{\vee}\right)}(1)\right), H:=\operatorname{pr}^{*} c_{1}\left(\mathscr{O}_{\mathbb{P}^{N}}(1)\right)$. По лемме 5 класс дивизоров $W+a_{0} H$ на $\mathbb{P}\left(E^{\vee}\right)$ обилен для достаточно большого $a_{0}-2+\delta_{B}(E)$. Более того, следствие из теоремы Клеймана (см. [11; следствие 1.4.9]) влечет обильность класса дивизоров

$$
\left(a_{0}-2+\delta_{B}(E)\right) c_{1}\left(L_{0}\right)+W+a_{0} H=\left(a_{0}-1+\delta_{B}(E)\right)\left(W+\left(2-\delta_{B}(E)\right) H\right) .
$$

Следовательно, $W+\left(2-\delta_{B}(E)\right) H$ также обилен.

Утверждение (ii) есть прямое следствие утверждения (i).

4.4. Напомним, что когерентный пучок $\mathscr{F}$ на $\mathbb{P}^{N} m$-регулярен в смысле Мамфорда-Кастельнуово, если $H^{i}(\mathscr{F}(m-i))=0$ для $i \geqslant 1$.

Теорема 5. Пусть Е-векторное расслоение ранга $r$ на $\mathbb{P}^{N}$.

(i) $E$ является $m$-регулярным при $m \geqslant m_{0}:=c_{1}(E)+(1+r)\left(2-\delta_{B}(E)\right)-1$. Более того, $E(m)$ порождается глобальными сечениями при $m \geqslant m_{0}$.

(ii) Для любой гиперплоскости $\mathbb{P}^{N-1}$ в $\mathbb{P}^{N}$ векторное расслоение $\left.E(m)\right|_{\mathbb{P}^{N-1}}$, $m \geqslant m_{0}$, порождается глобальными сечениями и

$$
h^{0}\left(\left.E(m)\right|_{\mathbb{P}^{N-1}}\right) \leqslant \frac{r}{(N-1) !}\left(\delta_{A}(E)+m+N-1\right)^{N-1} .
$$

ДокАЗАТЕЛЬСтво. Мы сохраняем обозначения лемм 7 и 8 . Дуализирующий пучок $\omega_{\mathbb{P}\left(E^{\vee}\right)}$ на $\mathbb{P}\left(E^{\vee}\right)$ дается стандартной формулой

$$
\omega_{\mathbb{P}\left(E^{\vee}\right)} \simeq \mathscr{O}_{\mathbb{P}\left(E^{\vee}\right)}(-r) \otimes \operatorname{pr}^{*} \mathscr{O}_{\mathbb{P}^{N}}\left(c_{1}(E)-N-1\right) .
$$


Поэтому (4.9) и (4.11) влекут изоморфизм

$$
\omega_{\mathbb{P}\left(E^{\vee}\right)} \otimes A_{i} \simeq \mathscr{O}_{\mathbb{P}\left(E^{\vee}\right)}(1) \otimes \operatorname{pr}^{*} \mathscr{O}_{\mathbb{P}^{N}}\left(m_{0}-N+i\right) .
$$

Так как $A_{i}$ обилен для $i \geqslant 0$ по лемме 8 , то теорема Кодаиры об обращении в нуль дает

$0=H^{j}\left(\omega_{\mathbb{P}\left(E^{\vee}\right)} \otimes A_{i}\right)=H^{j}\left(\mathscr{O}_{\mathbb{P}_{\left(E^{\vee}\right)}}(1) \otimes \operatorname{pr}^{*} \mathscr{O}_{\mathbb{P}^{N}}\left(m_{0}-N+i\right)\right), \quad i \geqslant 0, \quad j \geqslant 1$.

Кроме того, очевидно,

$\operatorname{pr}_{*}\left(\omega_{\mathbb{P}\left(E^{\vee}\right)} \otimes A_{i}\right) \simeq E\left(m_{0}-N+i\right), \quad R^{j} \operatorname{pr}_{*}\left(\omega_{\mathbb{P}\left(E^{\vee}\right)} \otimes A_{i}\right)=0, \quad j \geqslant 1, \quad i \geqslant 0$.

Поэтому спектральная последовательность Лере

$$
E_{2}^{a b}=H^{a}\left(R^{b} \operatorname{pr}_{*}\left(\omega_{\mathbb{P}\left(E^{\vee}\right)} \otimes A_{i}\right)\right) \quad \Longrightarrow \quad H^{a+b}\left(\omega_{\mathbb{P}\left(E^{\vee}\right)} \otimes A_{i}\right)
$$

вырождается и дает (в силу (4.12)) $H^{j}\left(E\left(m_{0}-N+i\right)\right)=0, i \geqslant 0, j \geqslant 1$. Это показывает, что $E m$-регулярно для $m \geqslant m_{0}$. Факт, что из $m$-регулярности расслоения $E$ следует, что $E(m)$ порождается глобальными сечениями, хорошо известен (см. [13; лемма 1.7.2]). Утверждение (i) доказано.

Утверждение (ii) следует из теоремы Ле Потье-Симпсона (см. [13; лемма 3.3.2]) в которой берется $X=\mathbb{P}^{N}, \operatorname{deg}(X)=1, F=E(m), \nu=N-1$, $X_{\nu}=\mathbb{P}^{N-1}, X_{1}=\mathbb{P}^{1}, \mu_{\max }\left(\left.E(m)\right|_{\mathbb{P}^{1}}\right)=\delta_{A}(E(m))=\delta_{A}(E)+m$.

\section{§5. Оценка сверху для степени многообразия прямых максимального подскока через точку для векторного расслоения на $\mathbb{P}^{N}$}

5.1. Преобразование $L_{0}$ векторного расслоения $E$ при линейной проекции. Пусть

$$
p_{x}: \mathbb{P}^{N} \rightarrow \mathbb{P}^{N-1}
$$

- рациональная линейная проекция с центром в точке $x \in \mathbb{P}^{N}$, и пусть $\widetilde{\mathbb{P}}^{N}-$ замыкание в $\mathbb{P}^{N} \times \mathbb{P}^{N-1}$ графика проекции $p_{x}$. Имеем следующую очевидную диаграмму проекций:

$$
\mathbb{P}^{N} \stackrel{\sigma}{\leftarrow} \widetilde{\mathbb{P}}^{N} \stackrel{\pi}{\rightarrow} \mathbb{P}^{N-1}
$$

Всюду в этом параграфе $E$ будет обозначать векторное расслоение ранга $r$ на $\mathbb{P}^{N}$ с дополнительным условием

$$
\delta_{B}(E)=0
$$

Положим $L_{0}:=\pi_{*} \sigma^{*} E$.

Tеорема 6. (i) $L_{0}$ есть векторное расслоение ранга

$$
\rho_{0}:=\operatorname{rk} L_{0}=c_{1}(E)+r
$$


на $\mathbb{P}^{N-1}$, и его конструкция совместима с заменой базы, т.е. для любого расслоенного квадрата

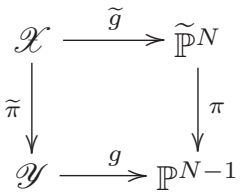

имеем изоморбизм замены базы

$$
\beta_{0}: \widetilde{\pi}_{*} \widetilde{g}^{*} \sigma^{*} E \stackrel{\sim}{\rightarrow} g^{*} \pi_{*} \sigma^{*} E=g^{*} L_{0} ;
$$

более того, естественное отображение вычисления еv: $\pi^{*} L_{0} \rightarrow \sigma^{*} E$ является эпиморфизмом.

(ii) $c_{1}\left(L_{0}\right)=P\left(c_{1}(E), c_{2}(E)\right)$, где $P(x, y):=\frac{1}{2} x(x+1)-y \in \mathbb{Q}[x, y]$.

(iii) $\delta_{A}(E) \geqslant \delta_{A}\left(L_{0}\right)$.

(iv) Выполняются неравенства

$$
\begin{gathered}
\delta_{A}\left(L_{0}\right) \geqslant-\left(P\left(c_{1}(E), c_{2}(E)\right)\right)^{2}, \\
\delta_{B}\left(L_{0}\right) \geqslant Q\left(r, \delta_{A}(E), c_{1}(E), c_{2}(E)\right),
\end{gathered}
$$

где $Q(x, y, z, t):=-(x+z) y+P(z, t)-(P(z, t))^{2} \in \mathbb{Q}[x, y]$ и многочлен $P$ определен в (ii).

ДокАЗАТЕльство. (i) Рассмотрим произвольную точку $y \in \mathbb{P}^{N-1}$ и положим $\mathbb{P}_{y}^{1}:=\pi^{-1}(y)$. Из $(5.2)$ немедленно получаем, что $h^{1}\left(\left.E\right|_{\mathbb{P}_{y}^{1}}\right)=0$, следовательно, $h^{0}\left(\left.E\right|_{\mathbb{P}_{y}^{1}}\right)=\chi\left(\left.E\right|_{\mathbb{P}_{y}^{1}}\right)=c_{1}(E)+r$. Эти равенства и теорема о замене базы (см. [8; ч. 3, теорема 12.11]) влекут (5.3), равенство $R^{1} \pi_{*} \sigma^{*} E=0$ и существование изоморфизма $\beta_{0}$. Более того, в силу $(5.2)$ пучок $\left.\sigma^{*} E\right|_{\mathbb{P}_{y}^{1}}$ порождается глобальными сечениями. Это означает, что существует эпиморфизм $\mathrm{ev}_{y}:\left.H^{0}\left(\left.\sigma^{*} E\right|_{\mathbb{P}_{y}^{1}}\right) \otimes \mathscr{O}_{\mathbb{P}_{y}^{1}} \rightarrow \sigma^{*} E\right|_{\mathbb{P}_{y}^{1}}$. Более того, отображение перехода к значению еv : $\pi^{*} L_{0} \rightarrow \sigma^{*} E$ совместимо с заменой базы, т.е. коммутативна диаграмма

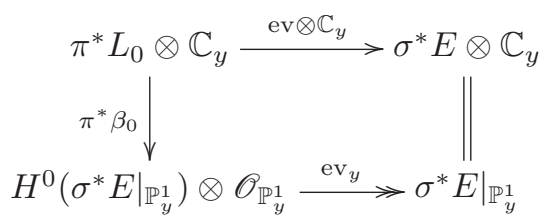

где $\pi^{*} \beta_{0}-$ изоморфизм; тем самым, отображение перехода к значению еv: $\pi^{*} L_{0} \rightarrow \sigma^{*} E$ эпиморфно.

(ii) На время доказательства фиксируем произвольную прямую $\mathbb{P}^{1} \subset \mathbb{P}^{N-1}$ и рассмотрим поверхность $S:=\pi^{-1}\left(\mathbb{P}^{1}\right)$ и проективную плоскость $\mathbb{P}^{2}:=\sigma(S)$ в $\mathbb{P}^{N}$. Эта плоскость проходит через центр $x$ проекции $p_{x}: \mathbb{P}^{N} \rightarrow \mathbb{P}^{N-1}$, и диаграмма (5.1) продолжается до коммутативной диаграммы

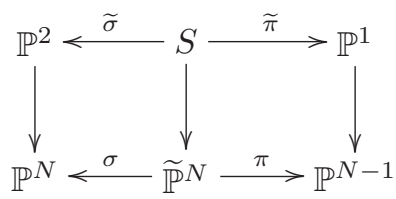


где $\tilde{\pi}=\left.\pi\right|_{S}, \tilde{\sigma}=\left.\sigma\right|_{S}: S \rightarrow \mathbb{P}^{2}$ есть раздутие $\mathbb{P}^{2}$ в точке $x$, а вертикальные стрелки являются вложениями.

Положим $\mathscr{O}_{S}(\tau):=\tilde{\sigma}^{*} \mathscr{O}_{\mathbb{P}^{2}}(1), \mathscr{O}_{S}(h):=\tilde{\pi}^{*} \mathscr{O}_{\mathbb{P}^{1}}(1)$. Заметим, что в кольце Чжоу $A(S)$ выполнены соотношения

$$
\tau^{2}=\tau h=[p t]
$$

Кроме того, $R^{1} \pi_{*} \sigma^{*} E=0$ влечет в силу замены базы равенство $\left.R^{1} \widetilde{\pi}_{*} \widetilde{\sigma}^{*} E\right|_{\mathbb{P}^{2}}=0$. Тем самым, теорема Римана-Роха (см., например, [12; пример 15.2.8]) дает

$$
\operatorname{ch}\left(\left.L_{0}\right|_{\mathbb{P}^{1}}\right)=\widetilde{\pi}_{*}\left(\operatorname{td}\left(T_{S / \mathbb{P}^{1}}\right) \cdot \operatorname{ch}\left(\left.\widetilde{\sigma}^{*} E\right|_{\mathbb{P}^{2}}\right)\right) .
$$

Здесь $T_{S / \mathbb{P} 1} \simeq \mathscr{O}_{S}(2 \tau-h)$. Поэтому, полагая $c_{i}:=c_{i}(E), i=1,2$, и используя соотношения (5.9), мы получаем в $A(S)$

$$
\operatorname{td}\left(T_{S / \mathbb{P} 1}\right) \cdot \operatorname{ch}\left(\left.\widetilde{\sigma}^{*} E\right|_{\mathbb{P}^{2}}\right)=1+\left(r+c_{1}\right) \tau-\frac{1}{2} r h+P\left(c_{1}, c_{2}\right)[p t] .
$$

Соответственно, в $A\left(\mathbb{P}^{1}\right)$ имеем

$$
\tilde{\pi}_{*}\left(\operatorname{td}\left(T_{S / \mathbb{P}^{1}}\right) \cdot \operatorname{ch}\left(\left.\widetilde{\sigma}^{*} E\right|_{\mathbb{P}^{2}}\right)\right)=\left(r+c_{1}\right) \cdot 1+P\left(c_{1}, c_{2}\right)[p t] .
$$

Отсюда следует (ii).

(iii) Положим $\widetilde{E}:=E\left(-\delta_{A}(E)-1\right)$. Тогда $\delta_{A}(\widetilde{E})=-1$ и

$$
\pi_{*} \sigma^{*} \widetilde{E}=0, \quad h^{0}(\widetilde{E})=h^{0}\left(\sigma^{*} \widetilde{E}\right)=0 .
$$

Вдобавок $(5.2)$ и условие $-1=\delta_{A}(\widetilde{E}) \geqslant \delta_{B}(\widetilde{E})$ дают

$$
D:=\delta_{A}(E)+1=-\delta_{B}(\widetilde{E})>0 .
$$

Пусть $l_{0}=\tilde{\sigma}^{-1}(x)$ - исключительная прямая на $S$. Так как $\mathscr{O}_{S}\left(l_{0}\right)=\mathscr{O}_{S}(\tau-h)$, имеем точную тройку

$$
0 \rightarrow \mathscr{O}_{S} \rightarrow \mathscr{O}_{S}(D(\tau-h)) \rightarrow \mathscr{O}_{D l_{0}}\left(D l_{0}\right) \rightarrow 0
$$

Здесь $D l_{0}$ обозначает стандартную схемную структуру кратности $D$ на $l_{0}$, как на дивизоре в $S$, и мы имеем точную тройку пучков $\mathscr{O}_{D l_{0}}$-модулей для $D \geqslant 2$

$$
\begin{aligned}
& 0 \rightarrow \mathscr{O}_{l_{0}}(-1) \rightarrow \mathscr{O}_{D l_{0}}\left(D l_{0}\right) \rightarrow \mathscr{O}_{(D-1) l_{0}}\left(D l_{0}\right) \rightarrow 0, \\
& 0 \rightarrow \mathscr{O}_{l_{0}}(-2) \rightarrow \mathscr{O}_{(D-1) l_{0}}\left(D l_{0}\right) \rightarrow \mathscr{O}_{(D-2) l_{0}}\left(D l_{0}\right) \rightarrow 0, \\
& 0 \rightarrow \mathscr{O}_{l_{0}}(1-D) \rightarrow \mathscr{O}_{2 l_{0}}\left(D l_{0}\right) \rightarrow \mathscr{O}_{l_{0}}(-D) \rightarrow 0 .
\end{aligned}
$$

Заметим, что по аналогии с (5.13)

$$
h^{0}\left(\left.\widetilde{\sigma}^{*} \widetilde{E}\right|_{\mathbb{P}^{2}}\right)=0 .
$$

Далее, поскольку $l_{0}=\widetilde{\sigma}^{-1}(x)$, то $\left.\left(\left.\widetilde{\sigma}^{*} \widetilde{E}\right|_{\mathbb{P}^{2}}\right)\right|_{m l_{0}} \simeq \mathscr{O}_{m l_{0}}^{r}$ для $m \geqslant 1$. Поэтому, подкручивая тройки $(5.16)$ на $\left.\widetilde{\sigma}^{*} \widetilde{E}\right|_{\mathbb{P}^{2}}$, мы получаем при $D \geqslant 2$

$$
h^{0}\left(\left.\widetilde{\sigma}^{*} \widetilde{E}\right|_{\mathbb{P}^{2}} \otimes \mathscr{O}_{D l_{0}}\left(D l_{0}\right)\right)=0 .
$$


Более того, равенство (5.18) очевидно при $D=1$, а значит, оно выполняется при $D \geqslant 1$.

Подкручивая $(5.15)$ на $\left.\widetilde{\sigma}^{*} \widetilde{E}\right|_{\mathbb{P}^{2}}$, мы получаем точную тройку

$$
\left.\left.\left.0 \rightarrow \widetilde{\sigma}^{*} \widetilde{E}\right|_{\mathbb{P}^{2}} \rightarrow \widetilde{\sigma}^{*} \widetilde{E}\right|_{\mathbb{P}^{2}}(D(\tau-h)) \rightarrow \widetilde{\sigma}^{*} \widetilde{E}\right|_{\mathbb{P}^{2}} \otimes \mathscr{O}_{D l_{0}}\left(D l_{0}\right) \rightarrow 0,
$$

и $(5.17)$ и (5.18) дают

$$
0=h^{0}\left(\left.\widetilde{\sigma}^{*} \widetilde{E}\right|_{\mathbb{P}^{2}}\right)(D(\tau-h))=h^{0}\left(\left.\widetilde{\pi}_{*} \widetilde{\sigma}^{*} \widetilde{E}\right|_{\mathbb{P}^{2}}(D(\tau-h))\right) .
$$

Применяя изоморфизм замены базы $\beta_{0}$ к правому квадрату диаграммы (5.8) и используя формулу проекции, получаем изоморфизмы

$$
\left.\widetilde{\pi}_{*}\left(\left.\widetilde{\sigma}^{*} \widetilde{E}\right|_{\mathbb{P}^{2}}(D(\tau-h))\right) \simeq\left(\left.\pi_{*} \sigma^{*} \widetilde{E}(D)\right|_{\mathbb{P}^{1}}\right)(-D) \simeq\left(\left.\pi_{*} \sigma^{*} E\right|_{\mathbb{P}^{1}}\right)(-D) \simeq L_{0}(-D)\right|_{\mathbb{P}^{1}} .
$$

Поэтому (5.19) влечет равенство $h^{0}\left(\left.L_{0}(-D)\right|_{\mathbb{P}^{1}}\right)=0$, т.е. $\delta_{A}\left(L_{0}\right)<D$, поскольку $\mathbb{P}^{1}$ есть произвольная прямая в $\mathbb{P}^{N-1}$. В совокупности с (5.14) это дает (iii).

(iv) Пусть

$$
\left.L_{0}\right|_{\mathbb{P}^{1}} \simeq \bigoplus_{i=1}^{\rho_{0}} \mathscr{O}_{\mathbb{P}^{1}}\left(a_{i}\right) .
$$

Ясно, что $\delta_{A}\left(L_{0}\right) \geqslant c_{1}\left(L_{0}\right) / \rho_{0}$, поскольку $a_{i} \leqslant \delta_{A}\left(L_{0}\right)$ для $1 \leqslant i \leqslant \rho_{0}$. Очевидно также, что $c_{1}\left(L_{0}\right) / \rho_{0} \geqslant-\left(c_{1}\left(L_{0}\right)\right)^{2}$. Поэтому $\delta_{A}\left(L_{0}\right) \geqslant-\left(c_{1}\left(L_{0}\right)\right)^{2}$. С другой стороны, согласно (ii) $-\left(c_{1}\left(L_{0}\right)\right)^{2}=-\left(P\left(c_{1}, c_{2}\right)\right)^{2}$. Поэтому выполняется (5.5).

Наконец, положим $\widetilde{L}_{0}:=L_{0}\left(-\delta_{A}\left(L_{0}\right)-1\right)$. Имеем

$$
\delta_{A}\left(\widetilde{L}_{0}\right)=-1, \quad \delta_{B}\left(L_{0}\right)=\delta_{B}\left(\widetilde{L}_{0}\right)+\delta_{A}\left(L_{0}\right)+1 .
$$

Предположим вдобавок, что прямая $\mathbb{P}^{1}$ в $\mathbb{P}^{N-1}$ выбрана таким образом, что $\delta_{B}\left(\left.\widetilde{L}_{0}\right|_{\mathbb{P}^{1}}\right)=\delta_{B}\left(\widetilde{L}_{0}\right)$. Тогда $h^{0}\left(\left.\widetilde{L}_{0}\right|_{\mathbb{P}^{1}}\right)=0$, и поэтому теорема Римана-Роха дает

$$
h^{1}\left(\left.\widetilde{L}_{0}\right|_{\mathbb{P}^{1}}\right)=-c_{1}\left(\widetilde{L}_{0}\right)-\operatorname{rk} \widetilde{L}_{0}=-c_{1}\left(\widetilde{L}_{0}\right)-\rho_{0} .
$$

С другой стороны, поскольку $\delta_{B}\left(\widetilde{L}_{0}\right) \leqslant \delta_{A}\left(\widetilde{L}_{0}\right)=-1$, мы имеем $-\delta_{B}\left(\widetilde{L}_{0}\right)-1=$ $h^{1}\left(\mathscr{O}_{\mathbb{P}^{1}}\left(\delta_{B}\left(\widetilde{L}_{0}\right)\right)\right) \leqslant h^{1}\left(\left.\widetilde{L}_{0}\right|_{\mathbb{P}^{1}}\right)$. Два последних неравенства вместе с $(5.21)$ влекут

$$
-1 \geqslant \delta_{B}\left(\widetilde{L}_{0}\right) \geqslant c_{1}\left(\widetilde{L}_{0}\right)+\rho_{0}-1 .
$$

Кроме того, определение $\widetilde{L}_{0}$ и утверждения (ii) и (iii) дают

$$
\begin{aligned}
c_{1}\left(\widetilde{L}_{0}\right)+\rho_{0}-1 & =-\rho_{0}\left(\delta_{A}\left(L_{0}\right)+1\right)+c_{1}\left(L_{0}\right)+\rho_{0}-1 \\
& \geqslant-\rho_{0}\left(\delta_{A}(E)+1\right)+P\left(c_{1}, c_{2}\right)+\rho_{0}-1 .
\end{aligned}
$$

Подставляя это вместе с (5.22) в (5.20) и используя (5.5) и (5.3), мы получаем неравенство

$\delta_{B}\left(L_{0}\right) \geqslant-\rho_{0} \delta_{A}(E)+P\left(c_{1}, c_{2}\right)+\delta_{A}\left(L_{0}\right) \geqslant-\left(r+c_{1}\right) \delta_{A}(E)+P\left(c_{1}, c_{2}\right)-\left(P\left(c_{1}, c_{2}\right)\right)^{2}$, т.е. неравенство (5.6). 
5.2. Оценка на преобразованное ядро отображения ev: $\pi^{*} L_{0} \rightarrow \sigma^{*} E$. Предположим вдобавок, что

$$
\delta_{A}(E)=2 \delta, \quad c_{1}(E)=r \delta
$$

для некоторого $\delta \in \mathbb{Z}_{>0}$. Положим

$$
\gamma:=c_{2}(E)-\frac{1}{2} r(r-1) \delta^{2} .
$$

Тогда теорема 6 дает

$$
\begin{gathered}
\rho_{0}=\operatorname{rk} L_{0}=r(1+\delta), \\
c_{1}\left(L_{0}\right)=P_{1}(r, \gamma, \delta), \\
Q_{1}(r, \gamma, \delta) \leqslant \delta_{B}\left(L_{0}\right) \leqslant \delta_{A}\left(L_{0}\right) \leqslant 2 \delta,
\end{gathered}
$$

где

$$
P_{1}(r, \gamma, \delta):=P\left(r \delta, \gamma+\frac{r(r-1) \delta^{2}}{2}\right), \quad Q_{1}(r, \gamma, \delta):=Q\left(r, 2 \delta, r \delta, \gamma+\frac{r(r-1) \delta^{2}}{2}\right) .
$$

По теореме 6, (i) мы имеем точную тройку векторных расслоений

$$
0 \rightarrow F \stackrel{\iota}{\rightarrow} \pi^{*} L_{0} \stackrel{\mathrm{ev}}{\rightarrow} \sigma^{*} E \rightarrow 0
$$

где $F:=$ Ker ev. Ограничение этой тройки на $S$ дает точную тройку

$$
\left.\left.0 \rightarrow F\right|_{S} \rightarrow \tilde{\pi}^{*}\left(\left.L_{0}\right|_{\mathbb{P}^{1}}\right) \rightarrow \tilde{\sigma}^{*} E\right|_{\mathbb{P}^{2}} \rightarrow 0
$$

и ее подкрученный вариант

$$
\left.0 \rightarrow\left(\left.F\right|_{S}\right)(j h) \rightarrow \tilde{\pi}^{*}\left(\left.L_{0}\right|_{\mathbb{P}^{1}}(j)\right) \rightarrow \tilde{\sigma}^{*} E\right|_{\mathbb{P}^{2}}(j h) \rightarrow 0 .
$$

Замена базы влечет изоморфизм $\left.\left.L_{0}\right|_{\mathbb{P}^{1}} \simeq \widetilde{\pi}_{*} \widetilde{\sigma}^{*} E\right|_{\mathbb{P}^{2}}$. Поэтому

$$
\left.L_{0}\right|_{\mathbb{P}^{1}}(j) \simeq\left(\left.\tilde{\pi}_{*} \tilde{\sigma}^{*} E\right|_{\mathbb{P}^{2}}\right)(j h), \quad j \in \mathbb{Z} .
$$

Поскольку $H^{1}\left(\tilde{\pi}^{*}\left(\left.L_{0}\right|_{\mathbb{P}^{1}}(j)\right)\right)=H^{1}\left(\left.L_{0}\right|_{\mathbb{P}^{1}}(j)\right)=0, j \geqslant-\delta_{B}\left(L_{0}\right)$, а морфизм $H^{0}\left(\tilde{\pi}^{*}\left(\left.L_{0}\right|_{\mathbb{P}^{1}}(j)\right)\right) \rightarrow H^{0}\left(\left.\widetilde{\sigma}^{*} E\right|_{\mathbb{P}^{2}}(j h)\right), j \in \mathbb{Z}$, индуцированный тройкой (5.30), является изоморфизмом, мы получаем

$$
h^{1}\left(\left.F\right|_{S}(j h)\right)=0, \quad j \geqslant-Q_{1}(r, \gamma, \delta)
$$

(см. (5.27)).

Далее, тройка (5.29) в силу (5.25), (5.26) влечет

$$
\begin{gathered}
\operatorname{rk} F=\operatorname{rk}\left(\left.F\right|_{S}\right)=r \delta, \\
c_{1}\left(\left.F\right|_{S}\right)=(r \delta) \tau-P_{1}(r, \gamma, \delta) h, \quad c_{2}\left(\left.F\right|_{S}\right)=P_{2}(r, \gamma, \delta)[p t],
\end{gathered}
$$

где $P_{2}(r, \gamma, \delta):=-\gamma-r(r-1) \delta^{2} / 2-r^{2} \delta^{2}+r \delta P_{1}(r, \gamma, \delta)$ и использованы соотношения (5.9). 
Положим

$$
b:=-\min \left\{\delta_{B}\left(\left.F\right|_{\mathbb{P}_{y}^{1}}\right) \mid y \in \mathbb{P}^{N-1}\right\}
$$

и отметим, что $b \geqslant 0$ в силу мономорфизма $\iota$ в (5.28). Для получения оценки сверху на $b$ возьмем точку $y \in \mathbb{P}^{N-1}$ такую, что $\delta_{B}\left(\left.F\right|_{\mathbb{P}_{y}^{1}}\right)=-b$. Тогда

$$
\left.F\right|_{\mathbb{P}_{y}^{1}} \simeq \bigoplus_{i=1}^{r \delta} \mathscr{O}_{\mathbb{P}^{1}}\left(b_{i}\right)=\mathscr{O}_{\mathbb{P}^{1}}(-b) \oplus \bigoplus_{i=1}^{r \delta} \mathscr{O}_{\mathbb{P}^{1}}\left(b_{i}\right),
$$

где $-b=b_{1} \leqslant b_{2} \leqslant \cdots \leqslant b_{r \delta} \leqslant 0$. Ограничивая (5.28) на $\mathbb{P}_{y}^{1}$ и используя $(5.25)$, мы получаем тройку

$$
\left.\left.0 \rightarrow F\right|_{\mathbb{P}_{y}^{1}} \rightarrow \mathscr{O}_{\mathbb{P}_{y}^{1}}^{r(1+\delta)} \rightarrow \sigma^{*} E\right|_{\mathbb{P}_{y}^{1}} \rightarrow 0
$$

Более того, (5.23) дает $\chi\left(\left.\sigma^{*} E\right|_{\mathbb{P}_{y}^{1}}\right)=\mathrm{rk} E+c_{1}(E)=r(1+\delta)$. Поэтому $0=$ $\chi\left(\left.F\right|_{\mathbb{P}_{y}^{1}}\right)=-b+\sum_{i=2}^{r \delta} b_{i}+r(1+\delta)$. Поскольку $\sum_{i=2}^{r \delta} b_{i} \leqslant 0$, мы получаем следующую оценку сверху на $b$ :

$$
b=\sum_{i=2}^{r \delta} b_{i}+r(1+\delta) \leqslant r(1+\delta) .
$$

Рассмотрим векторные расслоения

$$
\mathscr{O}_{\pi}(1):=\sigma^{*} \mathscr{O}_{\mathbb{P}^{N}}(1), \quad F_{b}:=F \otimes \mathscr{O}_{\pi}(b), \quad L_{1}:=\pi_{*} F_{b} .
$$

Заметим, что

$$
R^{1} \pi_{*} F_{b}=0
$$

Далее, (5.32) влечет равенства

$$
\begin{gathered}
c_{1}\left(\left.F_{b}\right|_{S}\right)=r \delta(1+b) \tau-h P_{1}(r, \gamma, \delta), \\
c_{2}\left(\left.F_{b}\right|_{S}\right)=c_{2}\left(\left.F\right|_{S}\right)+(r \delta-1)\left(r \delta \tau-h P_{1}(r, \gamma, \delta)\right) b \tau+\frac{1}{2} r b^{2} \delta(r \delta-1)[p t] .
\end{gathered}
$$

Замена базы вместе с (5.36) дает

$$
\tilde{\pi}_{*}\left(\left.F_{b}\right|_{S}\right)=\left.L_{1}\right|_{\mathbb{P}^{1}}, \quad R^{1} \tilde{\pi}_{*}\left(\left.F_{b}\right|_{S}\right)=0 .
$$

Следовательно, по теореме Римана-Роха (ср. (5.10))

$$
\operatorname{ch}\left(L_{1}{\mid \mathbb{P}^{1}}\right)=\tilde{\pi}_{*}\left(\operatorname{td}\left(T_{S / \mathbb{P}^{1}}\right) \cdot \operatorname{ch}\left(\left.F_{b}\right|_{S}\right)\right) .
$$

Подставляя (5.37) и (5.38) в (5.40) и продолжая, как при выводе равенств (5.11) и (5.12), мы получаем

$$
\begin{gathered}
\operatorname{rk} L_{1}=r \delta(2+b), \\
c_{1}\left(L_{1}\right)=F_{1}(r, b, \gamma, \delta):=\left(\begin{array}{c}
r \delta(1+b)+1 \\
2
\end{array}\right)-(r \delta(1+b)+1) P_{1}(r, \gamma, \delta)-F(r, b, \gamma, \delta) .
\end{gathered}
$$


Более того, (5.39) влечет равенства

$$
\tilde{\pi}_{*}\left(\left.F_{b}\right|_{S}(j h)\right)=\left.L_{1}(j)\right|_{\mathbb{P}^{1}}, \quad R^{1} \widetilde{\pi}_{*}\left(\left.F_{b}\right|_{S}(j h)\right)=0, \quad j \in \mathbb{Z} .
$$

Поэтому спектральная последовательность Лере $E_{2}^{a a^{\prime}}=H^{a}\left(R^{a^{\prime}} \widetilde{\pi}_{*}\left(\left.F_{b}\right|_{S}(j h)\right) \Rightarrow\right.$ $H^{a+a^{\prime}}\left(\left.F_{b}\right|_{S}(j h)\right)$ вырождается и

$$
H^{1}\left(\left.F_{b}\right|_{S}(j h)\right)=H^{1}\left(\left.L_{1}(j)\right|_{\mathbb{P}^{1}}\right), \quad j \in \mathbb{Z} .
$$

Теперь перейдем к доказательству следующей леммы.

Лемма 9. Существуют многочлены $R(x, y, z)$ u $S(x, y, z)$ в $\mathbb{Z}[x, y, z]$ такие, что

$$
-R(r, \gamma, \delta) \leqslant \delta_{B}\left(L_{1}\right) \leqslant \delta_{A}\left(L_{1}\right) \leqslant S(r, \gamma, \delta) .
$$

ДокАЗАТЕЛЬство. Зафиксируем прямую $l \subset \mathbb{P}^{2}$, не проходящую через точку $x$ (напомним, что $x$ есть центр раздутия $\tilde{\sigma}: S \rightarrow \mathbb{P}^{2}$ ), и пусть $\mathbb{P}_{\tau}^{1}:=\widetilde{\sigma}^{-1}(l)$. Тогда $\mathscr{O}_{S}\left(\mathbb{P}_{\tau}^{1}\right) \simeq \mathscr{O}_{S}(\tau)$. Ограничивая тройку (5.30) на $\mathbb{P}_{\tau}^{1}$, мы получаем точную тройку на $\mathbb{P}_{\tau}^{1} \simeq \mathbb{P}^{1}$

$$
\left.\left.0 \rightarrow F\right|_{\mathbb{P}_{\tau}^{1}}(j) \rightarrow \tilde{\pi}^{*}\left(\left.L_{0}\right|_{\mathbb{P}^{1}}(j)\right)\right|_{\mathbb{P}_{\tau}^{1}} \rightarrow \tilde{\sigma}^{*}\left(\left.E(j)\right|_{l}\right) \rightarrow 0 .
$$

Поскольку $\left.\left.\tilde{\pi}^{*}\left(\left.L_{0}\right|_{\mathbb{P}^{1}}(j)\right)\right|_{\mathbb{P}_{\tau}^{1}} \simeq L_{0}\right|_{\mathbb{P}^{1}}(j)$, мы имеем $\chi\left(\left.E(j)\right|_{l}\right)=r(1+\delta+j)$. Более того, $(5.25),(5.26)$ и теорема Римана-Роха дают

$$
\chi\left(\left.L_{0}\right|_{\mathbb{P}^{1}}(j)\right)=P_{1}(r, \gamma, \delta)+r(1+\delta)(j+1) .
$$

Следовательно,

$$
\chi\left(\left.F\right|_{\mathbb{P}_{\tau}^{1}}(j)\right)=P_{1}(r, \gamma, \delta)+r \delta j .
$$

Далее, (5.27) влечет

$$
Q_{1}(r, \gamma, \delta)+j \leqslant \delta_{B}\left(\left.L_{0}\right|_{\mathbb{P}^{1}}(j)\right) \leqslant \delta_{A}\left(\left.L_{0}\right|_{\mathbb{P}^{1}}(j)\right) \leqslant 2 \delta+j .
$$

С другой стороны, $\left.F\right|_{\mathbb{P}_{\tau}^{1}}(j) \simeq \bigoplus_{i=1}^{r \delta} \mathscr{O}_{\mathbb{P}^{1}}\left(e_{i}\right)$, где $\delta_{B}\left(\left.F\right|_{\mathbb{P}_{\tau}^{1}}(j)\right)=e_{1} \leqslant e_{2} \leqslant \ldots \leqslant e_{r \delta}$. Поэтому (5.45) дает

$$
P_{1}(r, \gamma, \delta)+r \delta j=\chi\left(\left.F\right|_{\mathbb{P}_{\tau}^{1}}(j)\right)=\delta_{B}\left(\left.F\right|_{\mathbb{P}_{\tau}^{1}}(j)\right)+r \delta+\sum_{i=2}^{r \delta} e_{i} .
$$

Заметим, что поскольку $\left.F\right|_{\mathbb{P}_{\tau}^{1}}(j)$ - подрасслоение в $\left.\left.\widetilde{\pi}^{*}\left(\left.L_{0}\right|_{\mathbb{P}^{1}}(j)\right)\right|_{\mathbb{P}_{\tau}^{1}} \simeq L_{0}\right|_{\mathbb{P}^{1}}(j)$, (5.46) влечет неравенства $e_{2} \leqslant \cdots \leqslant e_{r \delta} \leqslant \delta_{A}\left(\left.L_{0}(j)\right|_{\mathbb{P}^{1}}\right) \leqslant 2 \delta+j$, так что $\sum_{i=2}^{r \delta} e_{i} \leqslant(2 \delta+j)(r \delta-1)$. Эти неравенства вместе с (5.47) показывают, что

$$
\delta_{B}\left(\left.F\right|_{\mathbb{P}_{\tau}^{1}}(j)\right)=P_{1}(r, \gamma, \delta)+r \delta(j-1)-\sum_{i=2}^{r \delta} e_{i} \geqslant \delta(r-2 r \delta+2 \delta)-P_{1}(r, \gamma, \delta)+j .
$$

Следовательно,

$$
\delta_{B}\left(\left.F\right|_{\mathbb{P}_{\tau}^{1}}(j)\right) \geqslant 0, \quad j \geqslant P_{2}(r, \gamma, \delta):=P_{1}(r, \gamma, \delta)+\delta(-r+2 r \delta-2 \delta),
$$


откуда получаем

$$
j \geqslant P_{2}(r, \gamma, \delta) \quad \Longrightarrow \quad h^{1}\left(\left(\left.F\right|_{\mathbb{P}_{\tau}^{1}}\right)(j)\right)=0 .
$$

Рассмотрим теперь набор точных троек

$$
\begin{aligned}
& \left.\left.\left.0 \rightarrow F\right|_{S}(j h) \rightarrow F\right|_{S}(j h+\tau) \rightarrow F\right|_{\mathbb{P}_{\tau}^{1}}(j h+\tau) \rightarrow 0, \\
& \left.\left.\left.0 \rightarrow F\right|_{S}(j h+\tau) \rightarrow F\right|_{S}(j h+2 \tau) \rightarrow F\right|_{\mathbb{P}_{\tau}^{1}}(j h+2 \tau) \rightarrow 0, \\
& \left.\left.\left.0 \rightarrow F\right|_{S}(j h+(b-1) \tau) \rightarrow F\right|_{S}(j h+b \tau) \rightarrow F\right|_{\mathbb{P}_{\tau}^{1}}(j h+b \tau) \rightarrow 0,
\end{aligned}
$$

где

$$
j \geqslant \max \left\{-Q_{1}(r, \gamma, \delta), P_{2}(r, \gamma, \delta)\right\} \text {. }
$$

Поскольку $\left.\left.\mathscr{O}_{S}(\tau)\right|_{\mathbb{P}_{\tau}^{1}} \simeq \mathscr{O}_{S}(\tau)\right|_{\mathbb{P}_{\tau}^{1}} \simeq \mathscr{O}_{\mathbb{P}_{\tau}^{1}}(1)$, из (5.31) и (5.49) следует, что

$$
h^{1}\left(\left.F\right|_{S}(j h)\right)=h^{1}\left(\left.F\right|_{\mathbb{P}_{\tau}^{1}}(j h+i \tau)\right)=0, \quad 0 \leqslant i \leqslant b,
$$

для тех же значений $j$, что и в (5.50). Подставляя эти равенства в тройки из предыдущего набора и имея в виду, что $\left(\left.F\right|_{S}\right)(j h+b \tau)=\left(\left.F_{b}\right|_{S}\right)(j h)$, мы получаем в итоге

$$
h^{1}\left(\left.F_{b}\right|_{S}(j h)\right)=0, \quad j \geqslant \max \left\{-Q_{1}(r, \gamma, \delta), P_{2}(r, \gamma, \delta)\right\} .
$$

Положим $R(x, y, z):=\left(-Q_{1}(x, y, z)^{+}\right)+P_{2}(x, y, z)^{+}$(обозначение $(\cdot)^{+}$введено в $\S 2)$. Тогда $(5.43)$ и $(5.51)$ влекут равенства $h^{1}\left(\left.L_{1}(j)\right|_{\mathbb{P}^{1}}\right)=0, j \geqslant R(r, \gamma, \delta)$. Следовательно, поскольку $\mathbb{P}^{1}$ - произвольная прямая в $\mathbb{P}^{N-1}$, имеем

$$
-R(r, \gamma, \delta) \leqslant \delta_{B}\left(L_{1}\right) \text {. }
$$

Это дает левую часть неравенства (5.44).

Чтобы получить правую часть, рассмотрим прямую $\mathbb{P}^{1} \subset \mathbb{P}^{N-1}$ в диаграмме $(5.8)$ с $\delta_{A}\left(\left.L_{1}\right|_{\mathbb{P}^{1}}\right)=\delta_{A}\left(L_{1}\right)$ и

$$
\left.L_{1}\right|_{\mathbb{P}^{1}} \simeq \bigoplus_{i=1}^{r \delta(2+b)} \mathscr{O}_{\mathbb{P}^{1}}\left(a_{i}\right),
$$

где $\delta_{A}\left(L_{1}\right)=a_{1} \geqslant a_{2} \geqslant \cdots \geqslant a_{r \delta(2+b)} \geqslant \delta_{B}\left(L_{1}\right)$ и $\mathrm{rk} L_{1}=r \delta(2+b)$ в силу $(5.41)$. Заметим, что в силу $(5.41),(5.42)$ и теоремы Римана-Роха

$$
\chi\left(\left.L_{1}\right|_{\mathbb{P}^{1}}\right)=\operatorname{rk} L_{1}+c_{1}\left(L_{1}\right)=r \delta(2+b)+F_{1}(r, b, \gamma, \delta)=: F_{2}(r, b, \gamma, \delta) .
$$

С другой стороны,

$$
\chi\left(\left.L_{1}\right|_{\mathbb{P}^{1}}\right)=\delta_{A}\left(L_{1}\right)+\sum_{i=2}^{r \delta(2+b)} a_{i} .
$$

Отсюда в силу (5.52) мы получаем

$$
\begin{aligned}
\delta_{A}\left(L_{1}\right) & =\chi\left(\left.L_{1}\right|_{\mathbb{P}^{1}}\right)-\sum_{i=2}^{r \delta(2+b)} a_{i} \leqslant \chi\left(\left.L_{1}\right|_{\mathbb{P}^{1}}\right)-(r \delta(2+b)-1) \delta_{B}\left(L_{1}\right) \\
& \leqslant \chi\left(\left.L_{1}\right|_{\mathbb{P}^{1}}\right)+(r \delta(2+b)-1) R(r, \gamma, \delta) .
\end{aligned}
$$


Комбинируя эти неравенства с (5.53), находим

$$
\delta_{A}\left(L_{1}\right) \leqslant R_{1}(r, b, \gamma, \delta):=F_{2}(r, b, \gamma, \delta)+(r \delta(2+b)-1) R(r, \gamma, \delta) .
$$

Напомним, что в соответствии с (5.35)

$$
0 \leqslant b \leqslant r(1+\delta) .
$$

Полагая $S(x, y, z):=R_{1}(r, r(1+\delta), \gamma, \delta)^{+}$, мы получаем из (5.54) искомую правую часть неравенства (5.44).

5.3. Оценка для степени многообразия прямых максимального подскока $B_{\delta}^{\varkappa}\left(E^{0}, x, \mathbb{P}^{N-1}\right)$. Заметим, что из $(5.34)$ и $(5.33)$ вытекает, что $\left.F_{b}\right|_{\mathbb{P}_{y}^{1}}$ порождается глобальными сечениями для любой точки $y \in \mathbb{P}^{N-1}$. Поэтому замена базы дает эпиморфизм $\pi^{*} L_{1} \rightarrow F_{b}$ и его подкрутку

$$
\mathrm{ev}_{1}: \pi^{*} L_{1} \otimes \mathscr{O}_{\pi}(-b) \rightarrow F \text {. }
$$

Комбинируя (5.29) с (5.56), мы получаем точную последовательность

$$
\pi^{*} L_{1} \otimes \mathscr{O}_{\pi}(-b) \stackrel{\Psi}{\rightarrow} \pi^{*} L_{0} \rightarrow \sigma^{*} E \rightarrow 0,
$$

где $\Psi:=i \circ \mathrm{ev}_{1}$. Подкручивая (5.57) на $\pi$-относительный дуализирующий пучок и применяя $R^{1} \pi_{*}$, получаем точную последовательность

$$
L_{1} \otimes A_{b} \stackrel{\Phi}{\rightarrow} L_{0} \rightarrow R^{1} \pi_{*}\left(\sigma^{*} E \otimes \omega_{\pi}\right) \rightarrow 0,
$$

где

$$
\begin{aligned}
A_{b} & :=\left(\pi_{*} \mathscr{O}_{\pi}(b)\right)^{\vee} \simeq S^{b}\left(\mathscr{O}_{\mathbb{P}^{N-1}}(-1) \oplus \mathscr{O}_{\mathbb{P}^{N-1}}\right) \\
& =\mathscr{O}_{\mathbb{P}^{N-1}}(-b) \oplus \mathscr{O}_{\mathbb{P}^{N-1}}(-b+1) \oplus \cdots \oplus \mathscr{O}_{\mathbb{P}^{N-1}}
\end{aligned}
$$

Положим $E^{0}:=E(-\delta)$. Тогда $(5.23)$ и (5.24) дают

$$
c_{1}\left(E^{0}\right)=0, \quad c_{2}\left(E^{0}\right)=\gamma, \quad \delta_{A}\left(E^{0}\right)=\delta=-\delta_{B}\left(E^{0}\right) .
$$

Положим также

$$
B_{\delta}^{\varkappa}\left(E^{0}, x, \mathbb{P}^{N-1}\right):=\left\{y \in \mathbb{P}^{N-1} \mid \operatorname{dim}_{\mathbb{C}_{y}}\left(\mathbb{C}_{y} \otimes R^{1} \pi_{*}\left(\sigma^{*} E \otimes \omega_{\pi}\right)\right)=\varkappa\right\}
$$

для $x \in \mathbb{P}^{N}$.

Заметим, что $\varkappa \leqslant \mathrm{rk} E=r$. Поэтому в силу (5.25)

$$
r(1+\delta)=\rho_{0} \geqslant \rho_{0}-\varkappa \geqslant r \delta \geqslant 0 .
$$

Далее, обозначим

$$
\rho_{1}:=\operatorname{rk}\left(L_{1} \otimes A_{b}\right)=\operatorname{rk} L_{1} \cdot \operatorname{rk} A_{b}=r \delta(2+b)(1+b)
$$

(мы используем здесь (5.41) и (5.59)). Отметим, что (5.59) влечет равенства $\delta_{A}\left(L_{1} \otimes A_{b}\right)=\delta_{A}\left(L_{1}\right)-b, \delta_{B}\left(L_{1} \otimes A_{b}\right)=\delta_{B}\left(L_{1}\right)-b$, так что

$$
\begin{aligned}
j \delta_{B}\left(L_{1}\right)-j b & =j \delta_{B}\left(L_{1} \otimes A_{b}\right) \leqslant \delta_{B}\left(\wedge^{j}\left(L_{1} \otimes A_{b}\right)\right) \leqslant \delta_{A}\left(\wedge^{j}\left(L_{1} \otimes A_{b}\right)\right) \\
& \leqslant j \delta_{A}\left(L_{1} \otimes A_{b}\right)=j \delta_{A}\left(L_{1}\right)-j b
\end{aligned}
$$


для любого $j \in \mathbb{Z}_{>0}$. Это вместе с леммой 9 и (5.55) дает неравенства

$$
-j R(r, \gamma, \delta)-j r(1+\delta) \leqslant \delta_{B}\left(\wedge^{j}\left(L_{1} \otimes A_{b}\right)\right) \leqslant \delta_{A}\left(\wedge^{j}\left(L_{1} \otimes A_{b}\right)\right) \leqslant j S(r, \gamma, \delta) .
$$

Аналогичным образом (5.27) дает

$$
j Q_{1}(r, \gamma, \delta) \leqslant j \delta_{B}\left(L_{0}\right) \leqslant \delta_{B}\left(\wedge^{j} L_{0}\right) \leqslant \delta_{A}\left(\wedge^{j} L_{0}\right) \leqslant j \delta_{A}\left(L_{0}\right) \leqslant 2 j \delta .
$$

Заметим теперь, что, как показывает локально свободная резольвента (5.58) пучка $R^{1} \pi_{*}\left(\sigma^{*} E \otimes \omega_{\pi}\right)$, пучок $\varkappa$-х идеалов Фиттинга ${ }^{1} \mathscr{I}:=\mathscr{F}_{i t t}\left(R^{1} \pi_{*}\left(\sigma^{*} E \otimes\right.\right.$ $\left.\left.\omega_{\pi}\right)\right)$ пучка $R^{1} \pi_{*}\left(\sigma^{*} E \otimes \omega_{\pi}\right)$ совпадает с образом морфизма

$$
\Lambda: \mathscr{E}:=\wedge^{\left(\rho_{0}-\varkappa\right)}\left(L_{1} \otimes A_{b}\right) \otimes \Lambda^{\left(\rho_{0}-\varkappa\right)} L_{0}^{\vee} \rightarrow \mathscr{O}_{\mathbb{P}^{N-1}},
$$

индуцированного морфизмом $\Phi$ в (5.58). Таким образом, мы имеем эпиморфизм

$$
\mathscr{E} \rightarrow \mathscr{I}
$$

Обозначим через $V_{\delta}^{\varkappa}(x)$ подсхему в $\mathbb{P}^{N-1}$, определяемую пучком идеалов $\mathscr{I}$, т.e.

$$
\mathscr{O}_{V_{\delta}^{\varkappa}(x)}:=\mathscr{O}_{\mathbb{P}^{N-1}} / \mathscr{I}=\operatorname{coker} \Lambda .
$$

Тогда из (5.61) следует, что

$$
B_{\delta}^{\varkappa}\left(E^{0}, x, \mathbb{P}^{N-1}\right)=\operatorname{Supp}(\operatorname{coker} \Lambda)=V_{\delta}^{\varkappa}(x)_{\text {red }} .
$$

Ясно, что

$$
\begin{gathered}
\delta_{B}\left(\wedge^{\left(\rho_{0}-\varkappa\right)}\left(L_{1} \otimes A_{b}\right)\right)+\delta_{B}\left(\wedge^{\left(\rho_{0}-\varkappa\right)} L_{0}^{\vee}\right) \leqslant \delta_{B}(\mathscr{E}) \leqslant \delta_{A}(\mathscr{E}) \\
\leqslant \delta_{A}\left(\wedge^{\left(\rho_{0}-\varkappa\right)}\left(L_{1} \otimes A_{b}\right)\right)+\delta_{A}\left(\wedge^{\left(\rho_{0}-\varkappa\right)} L_{0}^{\vee}\right) .
\end{gathered}
$$

Подставляя сюда (5.64) и (5.65) с $j=\rho_{0}-\varkappa$ и используя (5.62), получаем

$$
T_{1}(r, \gamma, \delta) \leqslant \delta_{B}(\mathscr{E}) \leqslant \delta_{A}(\mathscr{E}) \leqslant T_{2}(r, \gamma, \delta)
$$

где

$$
\begin{gathered}
T_{1}(r, \gamma, \delta)=-r(1+\delta)\left(Q_{1}(r, \gamma, \delta)-R(r, \gamma, \delta)-r(1+\delta)\right)^{+} \\
T_{2}(r, \gamma, \delta)=r(1+\delta)(S(r, \gamma, \delta)+2 p \delta)^{+}
\end{gathered}
$$

Кроме того, принимая во внимание (5.25) и (5.63), находим

$$
\operatorname{rk} \mathscr{E}=I_{0}\left(r, b, \rho_{0}-\varkappa, \delta\right):=\left(\begin{array}{c}
r \delta(2+b)(1+b) \\
\rho_{0}-\varkappa
\end{array}\right)\left(\begin{array}{c}
r(1+\delta) \\
\rho_{0}-\varkappa
\end{array}\right) .
$$

Поэтому, используя (5.55) и (5.62), получаем

$$
\operatorname{rk} \mathscr{E} \leqslant I(r, \delta):=I_{0}(r, r(1+\delta), r(1+\delta), \delta)^{+} .
$$

\footnotetext{
${ }^{1}$ Определение пучков идеалов Фиттинга см., например, в [14; с. 492].
} 
Аналогично, (5.26) и (5.42) дают

$$
\begin{gathered}
c_{1}\left(\wedge^{\left(\rho_{0}-\varkappa\right)} L_{0}^{\vee}\right)=U_{0}(r, \gamma, \delta):=P_{1}(r, \gamma, \delta)\left(\begin{array}{c}
r(1+\delta)-1 \\
\rho_{0}-\varkappa-1
\end{array}\right), \\
c_{1}\left(\wedge^{\left(\rho_{0}-\varkappa\right)} L_{1} \otimes A_{b}\right)=U_{1}(r, b, \gamma, \delta) \\
:=(b+1)\left(F_{1}(r, b, \gamma, \delta)+\left(\begin{array}{c}
r(1+\delta)-1 \\
\rho_{0}-\varkappa-1
\end{array}\right) \frac{r \delta b(b+1)(b+2)}{2}\right) ;
\end{gathered}
$$

а значит,

$$
c_{1}(\mathscr{E})=J_{0}(r, b, \gamma, \delta):=U_{0}(r, \gamma, \delta) r \delta(2+b)(1+b)+U_{1}(r, \gamma, \delta) r(1+\delta)
$$

Тогда

$$
c_{1}(\mathscr{E}) \leqslant J(r, \gamma, \delta):=J_{0}(r, r(1+\delta), \gamma, \delta)^{+} .
$$

Применим теперь теорему 5 к векторному расслоению $\mathscr{E}$. Из $(5.69),(5.71)$ и (5.72) мы выводим, что $\mathscr{E}\left(m_{0}\right)$ глобально порождено для

$$
m_{0}=m_{0}(r, \gamma, \delta):=J(r, \gamma, \delta)+(1+I(r, \delta))\left(2-T_{1}(r, \gamma, \delta)\right)-1 .
$$

Таким образом, мы имеем эпиморфизм $\mathscr{O}_{\mathbb{P}^{N-1}}^{t_{0}} \rightarrow \mathscr{E}\left(m_{0}\right)$, где

$$
t_{0}=t_{0}(r, \gamma, \delta, N):=I(r, \delta)\left(T_{2}(r, \gamma, \delta)+m+N-1\right)^{N-1} .
$$

Следовательно, в силу (5.66) получаем эпиморфизм $\mathscr{O}_{\mathbb{P} N-1}^{t_{0}} \rightarrow \mathscr{I}\left(m_{0}\right)$. Этот эпиморфизм и теорема Безу показывают, что степень ${ }^{2}$ приведенной замкнутой подсхемы

$$
B_{\delta}^{\varkappa}\left(E^{0}, x, \mathbb{P}^{N-1}\right)=V_{\delta}^{\varkappa}(x)_{\text {red }}
$$

в $\mathbb{P}^{N-1}$ удовлетворяет неравенству

$$
\operatorname{deg} B_{\delta}^{\varkappa}\left(E^{0}, x, \mathbb{P}^{N-1}\right) \leqslant \operatorname{deg} V_{\delta}^{\varkappa}(x) \leqslant m_{0}^{t_{0}} .
$$

Подставляя сюда (5.73) и (5.74) и используя соотношения (5.60), получаем следующий основной результат этого параграфа.

Теорема 7. Пусть $E^{0}$ - векторное расслоение ранга $r$ на $\mathbb{P}^{N}$ с $c_{1}\left(E^{0}\right)=0$,

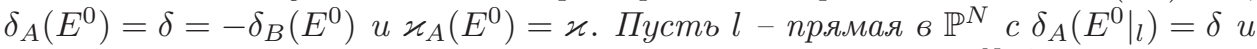
$\varkappa_{A}\left(\left.E^{0}\right|_{l}\right)=\varkappa$, и псть $x-$ произвольная точка на l. Пусть $\mathbb{P}^{N-1}$ - база семейства прямых через $x$ в $\mathbb{P}^{N}$. Тогда степень приведенной замкнутой подсхемы $B_{\delta}^{\varkappa}\left(E^{0}, x, \mathbb{P}^{N-1}\right)$ в $\mathbb{P}^{N-1}$ удовлетворяет неравенству

$$
\operatorname{deg} B_{\delta}^{\varkappa}\left(E^{0}, x, \mathbb{P}^{N-1}\right) \leqslant m_{0}\left(r, c_{2}\left(E^{0}\right), \delta\right)^{t_{0}\left(r, c_{2}\left(E^{0}\right), \delta, N\right)},
$$

где многочлены $m_{0}\left(x_{1}, x_{2}, x_{3}\right)$ и $t_{0}\left(x_{1}, x_{2}, x_{3}, x_{4}\right)$ задаются равенствами (5.73) и (5.74) соответственно.

\footnotetext{
${ }^{2}$ Под степенью замкнутой приведенной подсхемы в $\mathbb{P}^{N-1}$ мы понимаем сумму степеней ее неприводимых компонент.
} 


\section{§ 6. Доказательство теоремы 1}

6.1. В последующей части статьи мы фиксируем скрученный инд-грассманиан $\mathbf{G}=\underline{\lim } G\left(i_{m}, V^{n_{m}}\right)$, задаваемый последовательностью вложений $(1.1)$, и предполагаем, что $1<i_{m} \leqslant n_{m}-i_{m}$ для всех $m$. Положим $G_{m}:=G\left(i_{m}, V^{n_{m}}\right)$ и $\widetilde{\varphi}_{m}:=\varphi_{m-1} \circ \cdots \circ \varphi_{1}$. Мы фиксируем также самодвойственное векторное расслоение $\mathbf{E}=\lim _{m} E_{m}$ на $\mathbf{G}$ (последнее означает, что $E_{m} \simeq E_{m}^{\vee}$ для каждого $m$ ) ранга $\mathbf{r} \in \overleftarrow{\mathbb{Z}_{>0}}$. Тогда

$$
c_{1}\left(E_{m}\right)=0, \quad \delta\left(E_{m}\right)=2 \delta_{A}\left(E_{m}\right), \quad m \geqslant 1 .
$$

Заметим, что теорему 1 достаточно доказать для самодвойственных расслоений $\mathbf{E}$. В самом деле, рассмотрим произвольное векторное расслоение конечного ранга $\mathbf{E}^{\prime}=\lim _{m} E_{m}^{\prime}$ на $\mathbf{G}$. Положим $\mathbf{E}=\mathscr{E} n d \mathbf{E}^{\prime}$. Так как $\mathbf{E}$ самодвойственно, мы можем считать, что теорема 1 верна для $\mathbf{E}$. Поэтому для любого $m$ и любой прямой $l$ в $G\left(i_{m}, V^{n_{m}}\right)$ расслоение $\left.\left(\mathscr{E} n d E_{m}^{\prime}\right)\right|_{l}$ есть тривиальное расслоение. Теорема Гротендика для векторных расслоений на $\mathbb{P}^{1}$ немедленно влечет, что расслоение $\left.E_{m}^{\prime}\right|_{l} \otimes L_{m, l}$ является тривиальным расслоением для подходящего линейного расслоения $L_{m, l}$ на $l$. Так как $c_{1}\left(L_{m, l}\right)=-c_{1}\left(E_{m}^{\prime}\right) / \mathrm{rk} E_{m}^{\prime}$ не зависит от $l$, линейные расслоения $L_{m, l}$ определяют линейное расслоение $L_{m}$ на $G_{m}$. Теперь стандартный результат из [7] (предложение 1.4.1) показывает, что $E_{m}^{\prime} \otimes L_{m}$ тривиально для всех $m$. Поэтому $\lim _{\longleftarrow}\left(E_{m}^{\prime} \otimes L_{m}\right)$ тривиально. Чтобы увидеть, что $\mathbf{E}^{\prime}$ само является тривиальным, заметим, что линейные расслоения $L_{m}$ определяют линейное расслоение $\mathbf{L}=\lim L_{m}$ на $\mathbf{G}$. Поскольку $\mathbf{G}$ является скрученным грассманианом, то при любом $m$ класс Черна $c_{1}\left(\left.\mathbf{L}\right|_{G_{m}}\right)=c_{1}\left(L_{m}\right)$ должен делиться на $\operatorname{deg}\left(\varphi_{n} \circ \cdots \circ \varphi_{m}\right)$ для всех $n>m$. Так как $\lim _{n \rightarrow \infty} \operatorname{deg}\left(\varphi_{n} \circ \cdots \circ \varphi_{m}\right)=\infty$, то отсюда следует, что $c_{1}\left(L_{m}\right)=0$, и, следовательно, $\mathbf{L}$ тривиально. Поэтому $\mathbf{E}^{\prime} \simeq \underset{\lim }{\longleftarrow}\left(E_{m}^{\prime} \otimes L_{m}\right)$ тривиально.

6.2. Наблюдение о классе $c_{2}(\mathbf{E})$. Заметим, что вложения $\varphi_{m}: G_{m} \rightarrow$ $G_{m+1}$ определяют гомоморфизмы $\varphi_{m}^{*}: A^{2}\left(G_{m+1}\right) \rightarrow A^{2}\left(G_{m}\right)$, и второй класс Черна расслоения $\mathbf{E}$ есть по определению проективная система $\left\{c_{2}\left(E_{m}\right)=\right.$ $\left.\varphi_{m}^{*} c_{2}\left(E_{m+1}\right)\right\}_{m \geqslant 1}$. Здесь

$$
A\left(G_{m}\right)=\bigoplus_{i \geqslant 0} A^{i}\left(G_{m}\right)
$$

обозначает кольцо Чжоу грассманиана $G_{m}$, и мы напоминаем некоторые стандартные факты о кольце $A\left(G_{m}\right)$ (ср. $[12 ;$ гл. $\left.14, \S 7]\right)$ :

(i) $A^{1}\left(G_{m}\right)=\operatorname{Pic}\left(G_{m}\right)=\mathbb{Z}\left[\mathbb{V}_{m}\right], A^{2}\left(G_{m}\right)=\mathbb{Z}\left[\mathbb{W}_{1, m}\right] \oplus \mathbb{Z}\left[\mathbb{W}_{2, m}\right]$, где $\mathbb{V}_{m}, \mathbb{W}_{1, m}$, $\mathbb{W}_{2, m}$ - многообразия Шуберта:

$$
\begin{gathered}
\mathbb{V}_{m}=\left\{V^{i_{m}} \in G_{m} \mid \operatorname{dim}\left(V^{i_{m}} \cap V_{0}^{n_{m}-i_{m}-1}\right) \geqslant 1\right. \\
\mathbb{W}_{1, m}=\left\{V^{i_{m}} \in G_{m} \mid \operatorname{dim}\left(V^{i_{m}} \cap V_{0}^{n_{m}-i_{m}-1}\right) \geqslant 1\right. \\
\text { для фиксированного подпространства } \left.V_{0}^{n_{m}-i_{m}-1} \text { в } V^{n_{m}}\right\}, \\
\mathbb{W}_{2, m}=\left\{V^{i_{m}} \in G_{m} \mid \operatorname{dim}\left(V^{i_{m}} \cap V_{0}^{n_{m}-i_{m}+1}\right) \geqslant 2\right. \\
\text { для фиксированного подпространства } \left.V_{0}^{n_{m}-i_{m}+1} \text { в } V^{n_{m}}\right\} ;
\end{gathered}
$$


(ii) $\left[\mathbb{V}_{m}\right]^{2}=\left[\mathbb{W}_{1, m}\right]+\left[\mathbb{W}_{2, m}\right]$ в $A^{2}\left(G_{m}\right)$;

(iii) существуют целые числа $a_{i j}(m) \geqslant 0, i, j=1,2$, такие, что

$$
\begin{gathered}
\varphi_{m}^{*}\left[\mathbb{W}_{1, m+1}\right]=a_{11}(m)\left[\mathbb{W}_{1, m}\right]+a_{21}(m)\left[\mathbb{W}_{2, m}\right], \\
\varphi_{m}^{*}\left[\mathbb{W}_{2, m+1}\right]=a_{12}(m)\left[\mathbb{W}_{1, m}\right]+a_{22}(m)\left[\mathbb{W}_{2, m}\right], \\
a_{11}(m)+a_{12}(m)=a_{21}(m)+a_{22}(m)=\left(\operatorname{deg} \varphi_{m}\right)^{2}, \quad m \geqslant 1 .
\end{gathered}
$$

Лемма 10. Для данного расслоения $\mathbf{E}=\lim _{m} E_{m}$ существует бесконечная подпоследовательность последовательности грассманианов $G_{m}$ такая, что координаты класса $c_{2}\left(E_{m}\right)$ в базисе $\left\{\left[\mathbb{W}_{1, m}\right],\left[\mathbb{W}_{2, m}\right]\right\}$ являются константами $\lambda_{1} \in \mathbb{Z} u \lambda_{2} \in \mathbb{Z}$. Более того, если $\lambda_{1} \lambda_{2} \neq 0$, то $\lambda_{1} \lambda_{2}<0$.

ДокАЗАТЕЛЬСтво. Пусть

$$
c_{2}\left(E_{m}\right)=\lambda_{1 m}\left[\mathbb{W}_{1, m}\right]+\lambda_{2 m}\left[\mathbb{W}_{2, m}\right] .
$$

Рассмотрим $(2 \times 2)$-матрицу $A(m)=\left(a_{i j}(m)\right)$ и вектор-столбец $\Lambda_{m}=\left(\lambda_{1 m}, \lambda_{2 m}\right)^{t}$. Соотношения (6.4) и (6.2) дают

$$
\Lambda_{m}=A(m) \Lambda_{m+1}
$$

Положим $\gamma_{m}:=\lambda_{1 m}-\lambda_{2 m}$. Тогда, подставляя (6.3) в (6.5), находим

$$
\begin{gathered}
\gamma_{m}=\left(a_{11}(m)-a_{21}(m)\right) \gamma_{m+1}=\gamma_{m+m^{\prime}+1} \prod_{i=1}^{m^{\prime}}\left(a_{11}(m+i)-a_{21}(m+i)\right), \\
m, m^{\prime} \geqslant 1 .
\end{gathered}
$$

Предположим, что $\gamma_{m_{0}} \neq 0$ для некоторого $m_{0} \geqslant 1$. Тогда (6.6) влечет $a_{11}(m)-a_{21}(m) \neq 0, \gamma_{m} \neq 0, m \geqslant m_{0}$. Кроме того, если $\left|a_{11}(m)-a_{21}(m)\right|>1$ для бесконечного набора значений $m \geqslant m_{0}$, то правая часть в (6.6) неограниченно возрастает при $m^{\prime} \rightarrow \infty$, приводя к противоречию. Следовательно, $\left|a_{11}(m)-a_{21}(m)\right|>1$ для не более чем конечного числа значений $m \geqslant m_{0}$. Удаляя грассманианы $G_{m}$ с этими значениями $m$ из нашего инд-грассманиана $\mathbf{G}$ (и беря в качестве новых вложений соответствующие композиции старых вложений), мы можем считать, что $\left|a_{11}(m)-a_{21}(m)\right|=1$ для всех $m \geqslant m_{0}$. Поскольку для бесконечного числа значений $m$ числа $a_{11}(m)-a_{21}(m)$ имеют одинаковый знак, то последовательность $\left\{\gamma_{m}\right\}$ имеет бесконечную постоянную подпоследовательность. Следовательно, снова после удаления подходящих значений $m$ в конструкции инд-грассманиана $\mathbf{G}$ мы можем считать, что

$$
\gamma:=\gamma_{m}=\lambda_{1 m}-\lambda_{2 m} \neq 0, \quad m \geqslant m_{0} .
$$

Пусть $\gamma>0$. (В случае $\gamma<0$ рассуждения аналогичны.) Как показано в [7; $\S 5]$, при достаточно большом $m$ (скажем, при $m \geqslant m_{0}$ ) оба числа $\lambda_{1 m}$ и $\lambda_{2 m}$ не могут одновременно быть ненулевыми числами одного знака. (Доказательство в [7] проведено для расслоений ранга 2 , но оно годится для расслоений $E_{m}$ произвольного ранга.) Это свойство вместе с (6.7) влечет неравенства

$$
\gamma \geqslant \lambda_{1 m} \geqslant 0, \quad 0 \geqslant \lambda_{2 m} \geqslant-\gamma, \quad m \geqslant m_{0} .
$$


Таким образом, существуют бесконечные постоянные подпоследовательности $\left\{\lambda_{1, m^{\prime}}=: \lambda_{1} \geqslant 0\right\}_{m^{\prime} \geqslant m_{0}}$ и $\left\{\lambda_{2, m^{\prime}}=: \lambda_{2} \leqslant 0\right\}_{m^{\prime} \geqslant m_{0}}$ соответственно последовательностей $\left\{\lambda_{1 m}\right\}_{m \geqslant m_{0}}$ и $\left\{\lambda_{2 m}\right\}_{m \geqslant m_{0}}$. Поэтому снова без ограничения общности мы можем считать, что последовательности $\left\{\lambda_{1 m}\right\}_{m \geqslant m_{0}}$ и $\left\{\lambda_{2 m}\right\}_{m \geqslant m_{0}}$ постоянны:

$$
0 \leqslant \lambda_{1}=\lambda_{1 m}, \quad 0 \geqslant \lambda_{2}=\lambda_{2 m}, \quad m \geqslant m_{0} .
$$

Всюду ниже мы предполагаем, что координаты класса $c_{2}\left(E_{m}\right)$ в базисе $\left\{\left[\mathbb{W}_{1, m}\right],\left[\mathbb{W}_{2, m}\right]\right\}$ постоянны для нашей фиксированной последовательности грассманианов $G_{m}$.

Напомним, что имеются два семейства проективных подпространств максимальной размерности в $G_{m}$ : семейство $\mathbb{I}_{1}$, состоящее из подпространств

$$
\mathbb{P}^{i_{m}}=\left\{V^{i_{m}} \in G_{m} \mid V^{i_{m}} \subset V_{0}^{i_{m}+1}\right\}, \quad V_{0}^{i_{m}+1} \in G\left(i_{m}+1, V^{n_{m}}\right),
$$

и семейство $\mathbb{I}_{2}$, состоящее из подпространств

$$
\mathbb{P}^{n_{m}-i_{m}}=\left\{V^{i_{m}} \in G_{m} \mid V^{i_{m}} \supset V_{0}^{i_{m}-1}\right\}, \quad V_{0}^{i_{m}-1} \in G\left(i_{m}-1, V^{n_{m}}\right) .
$$

Лемма 10 влечет нижеследующее.

СлЕДСТвиЕ 1. В обозначениях леммы 10 мы имеем

$$
\begin{aligned}
c_{2}\left(\left.E_{m}\right|_{\mathbb{P}^{i m}}\right) & =\lambda_{2}, & & \mathbb{P}^{i_{m}} \in \mathbb{I}_{1}, \\
c_{2}\left(\left.E_{m}\right|_{\mathbb{P}^{n_{m}-i_{m}}}\right) & =\lambda_{1}, & & \mathbb{P}^{n_{m}-i_{m}} \in \mathbb{I}_{2} .
\end{aligned}
$$

\section{3. Многообразие прямых максимального подскока расслоения} $E_{m}$, проходящих через точку. Для фиксированного $m$ рассмотрим естественную диаграмму

$$
G_{m}=G\left(i_{m}, V^{n_{m}}\right) \stackrel{\pi_{1}}{\longleftarrow} \Gamma_{m} \stackrel{\pi_{2}}{\longrightarrow} \mathrm{Fl}_{m}
$$

где $\Gamma_{m}:=\mathrm{Fl}\left(i_{m}-1, i_{m}, i_{m}+1, V^{n_{m}}\right)$ и $\mathrm{Fl}_{m}:=\mathrm{Fl}\left(i_{m}-1, i_{m}+1, V^{n_{m}}\right)$ есть база семейства (проективных) прямых на $G_{m}$. Положим

$$
Z_{a}\left(E_{m}\right):=\left\{l \in \mathrm{Fl}_{m} \mid \delta_{A}\left(\left.E_{m}\right|_{l}\right) \geqslant a\right\}, \quad B_{a}\left(E_{m}\right):=\pi_{2}^{-1}\left(Z_{a}\left(E_{m}\right)\right), \quad a \in \mathbb{Z}_{>0} .
$$

Из полунепрерывности $\delta_{A}\left(\left.E_{m}\right|_{l}\right)$ как функции от $l$ следует, что $Z_{a}\left(E_{m}\right)$ замкнуто в $\mathrm{Fl}_{m}$; соответственно $B_{a}\left(E_{m}\right)$ замкнуто в $\Gamma_{m}$. Далее, положим

$$
\Delta:=\min \left\{a \mid \operatorname{im}\left(\pi_{1}\left(B_{a}\left(E_{m}\right)\right)\right) \neq G_{m}\right\}-1 .
$$

Тогда $Y:=\pi_{1}\left(B_{\Delta+1}\left(E_{m}\right)\right) \neq G_{m}, \pi_{1}\left(B_{\Delta}\left(E_{m}\right)\right)=G_{m}$ и множество $G_{m}^{\prime}:=G_{m} \backslash Y=\left\{x \in G_{m} \mid \Delta=\max \left\{\delta_{A}\left(\left.E_{m}\right|_{l}\right) \mid l\right.\right.$ есть прямая на $G_{m}$ через $\left.\left.x\right\}\right\}$ является плотным открытым подмножеством в $G_{m}$.

Обозначим $p_{\Delta, E_{m}}:=\left.\pi_{1}\right|_{B_{\Delta}\left(E_{m}\right)}$. Тогда множество $B_{\Delta}\left(E_{m}\right)^{\prime}:=p_{\Delta, E_{m}}^{-1}\left(G_{m}^{\prime}\right)$ замкнуто в $\pi_{1}^{-1}\left(G_{m}^{\prime}\right)$ и морфизм $p_{\Delta, E_{m}}: B_{\Delta}\left(E_{m}\right)^{\prime} \rightarrow G_{m}^{\prime}$ проективен и сюръективен. Аналогично, для каждого $a, 1 \leqslant a \leqslant \mathbf{r}$,

$$
B_{\Delta}^{a}\left(E_{m}\right):=\left\{(x, l) \in B_{\Delta}\left(E_{m}\right) \mid l \in Z_{\Delta}\left(E_{m}\right), \varkappa_{A}\left(\left.E_{m}\right|_{l}\right) \geqslant a\right\}
$$


является замкнутым подмножеством в $B_{\Delta}\left(E_{m}\right)$; соответственно множество $p_{\Delta, E_{m}}\left(B_{\Delta}^{a}\left(E_{m}\right)\right)$ замкнуто в $G_{m}$. Поскольку $\varkappa_{A}\left(\left.E_{m}\right|_{l}\right) \geqslant 1$ для любой $l \in$ $Z_{\Delta}\left(E_{m}\right)$, мы получаем, что $\pi_{1}\left(B_{\Delta}^{1}\left(E_{m}\right)\right)=G_{m}$.

Если $\pi_{1}\left(B_{\Delta}^{\mathrm{r}}\left(E_{m}\right)\right) \neq G_{m}$, мы полагаем

$$
\begin{aligned}
K & :=\min \left\{2 \leqslant a \leqslant \mathbf{r} \mid \pi_{1}\left(B_{\Delta}^{a}\left(E_{m}\right)\right) \neq G_{m}\right\}-1, \quad T:=\pi_{1}\left(B_{\Delta}^{K+1}\left(E_{m}\right)\right), \\
G_{m}^{0} & :=G_{m}^{\prime} \backslash T, \quad B_{\Delta}^{K}\left(E_{m}\right)^{0}:=\pi_{1}^{-1}\left(G_{m}^{0}\right) \cap B_{\Delta}^{K}\left(E_{m}\right)
\end{aligned}
$$

если $\pi_{1}\left(B_{\Delta}^{\mathbf{r}}\left(E_{m}\right)\right)=G_{m}$, то полагаем

$$
K:=\mathbf{r}, \quad G_{m}^{0}:=G_{m}^{\prime}, \quad B_{\Delta}^{K}\left(E_{m}\right)^{0}:=B_{\Delta}\left(E_{m}\right) .
$$

По определению $G_{m}^{0}$ есть плотное открытое подмножество в $G_{m}^{\prime}$, а значит, и в $G_{m}$, и морфизм $p_{\Delta, E_{m}}^{K}:=\left.\pi_{1}\right|_{B_{\Delta}^{K}\left(E_{m}\right)^{0}}: B_{\Delta}^{K}\left(E_{m}\right)^{0} \rightarrow G_{m}^{0}$ проективен и сюръективен.

6.4. Оценка коразмерности множества $B_{\Delta}^{K}\left(E_{m}\right)$. Полунепрерывность $\delta_{A}\left(\left.E_{m}\right|_{l}\right)$ (соответственно $\left.\delta\left(\left.E_{m}\right|_{l}\right)\right)$ как функции от $l$ показывает, что минимальное значение $\delta_{A}\left(\left.E_{m}\right|_{l}\right)$ (соответственно $\delta\left(\left.E_{m}\right|_{l}\right)$ ) достигается на плотном открытом множестве прямых $l \in \mathrm{Fl}_{m}$. В дальнейшем мы обозначаем это минимальное значение через $\delta_{A}^{\text {gen }}\left(E_{m}\right)$ (соответственно через $\delta^{\text {gen }}\left(E_{m}\right)$ ).

ЛЕмма 11. Предположим, что $\delta_{A}^{\text {gen }}\left(E_{m}\right)>\frac{1}{2} \mathbf{r}$. Тогда существует подnучoк $\mathscr{F}_{m}$ в $E_{m}$ c $c_{1}\left(\mathscr{F}_{m}\right)>0$.

ДокАзАтЕльство. Из неравенства $\delta_{A}^{\text {gen }}\left(E_{m}\right)>\frac{1}{2} \mathbf{r}$ и зануления $c_{1}\left(E_{m}\right)$ следует, что для любой прямой $l \subset G_{m}$ тип расщепления $\left(\delta_{1}, \ldots, \delta_{\mathbf{r}}\right)$ расслоения $\left.E\right|_{l}$ удовлетворяет неравенству $\delta_{s}-\delta_{s+1} \geqslant 2$ для некоторого $s, 1 \leqslant s \leqslant \mathbf{r}-1$. Поэтому выполнено условие теоремы 1.4.2 из [7] (являющейся вариантом леммы о спуске из [15; ч. II, лемма 2.1.2] для случая грассманианов), и эта теорема дает подпучок $\mathscr{F}_{m}$ в $E_{m}$. Поскольку $E_{m}$ самодвойственно, зануление класса $c_{1}\left(E_{m}\right)$ влечет положительность числа $\delta_{s}$, а значит, по построению пучка $\mathscr{F}_{m}$ мы имеем $c_{1}\left(\mathscr{F}_{m}\right)=\delta_{1}+\cdots+\delta_{s}>0$.

Лемма 12. Для достаточно большого $m$ не существует подпучков $\mathscr{F}_{m} \subset$ $E_{m} c c_{1}\left(\mathscr{F}_{m}\right)>0$.

ДокАЗАТЕльство. Пусть $\widetilde{d}_{m}:=\operatorname{deg} \widetilde{\varphi}_{m}$. Рассмотрим многочлен $P_{m}(t):=$ $\widetilde{d}_{m} t+1$, и положим

$$
\begin{gathered}
\mathscr{H}_{m}:=\left\{C \in \operatorname{Hilb}^{P_{m}(t)} G_{m} \mid C\right. \text { есть гладкая неприводимая кривая } \\
\text { степени } \left.\widetilde{d}_{m} \text { на } G_{m}\right\} .
\end{gathered}
$$

Из работы С. Стрёмме [16] известно, что $\mathscr{H}_{m}$ есть гладкое неприводимое многообразие размерности $n_{m} \widetilde{d}_{m}+i_{m}\left(n_{m}-i_{m}\right)-3$.

Предположим, что $\mathscr{F}_{m}$ есть подпучок в $E_{m}$ с $c_{1}\left(\mathscr{F}_{m}\right)>0$. Тогда

$$
\operatorname{codim}_{G_{m}} \operatorname{Sing} \mathscr{F}_{m} \geqslant 2 \text {, }
$$

поскольку $E_{m}$ локально свободен (см. [15; ч. II, следствие 1.1.9]). Более того, так как $G_{m}$ является однородным пространством, $\mathscr{H}_{m}^{*}:=\left\{C \in \mathscr{H}_{m} \mid C \cap\right.$ Sing $\mathscr{F}_{m}=\varnothing$ \} есть плотное открытое множество в $\mathscr{H}_{m}$. 
Положим $a_{m}:=\min _{C \in \mathscr{H}_{m}}\left\{\delta_{A}\left(\left.E_{m}\right|_{C}\right)\right\}$. Так как $\delta_{A}\left(\left.E_{m}\right|_{C}\right)$ полунепрерывна как функция от $C$, то $\mathscr{H}_{m}^{0}:=\left\{C \in \mathscr{H}_{m} \mid \delta_{A}\left(\left.E_{m}\right|_{C}\right)=a_{m}\right\}$ есть плотное открытое подмножество в $\mathscr{H}_{m}$ и для любой проективной прямой $l \subset G_{1}$

$$
\delta_{A}\left(\left.E_{1}\right|_{l}\right)=\delta_{A}\left(\left.E_{m}\right|_{C_{1}}\right) \geqslant a_{m},
$$

где $C_{1}:=\widetilde{\varphi}_{m}(l) \in \mathscr{H}_{m}$. Теперь допустим, что $c_{1}\left(\mathscr{F}_{m}\right) \geqslant 1$, и рассмотрим произвольную кривую $C \in \mathscr{H}_{m}^{*} \cap \mathscr{H}_{m}^{0}$ такую, что $\delta_{A}\left(\left.E_{m}\right|_{C}\right)=a_{m}$. Так как $\left.\mathscr{F}_{m}\right|_{C}$ является локально свободным подпучком в $\left.E_{m}\right|_{C}$ с $c_{1}\left(\mathscr{F}_{m}\right) \geqslant 1$, то, следовательно,

$$
a_{m}=\delta_{A}\left(\left.E_{m}\right|_{C}\right) \geqslant \delta_{A}\left(\left.\mathscr{F}_{m}\right|_{C}\right) \geqslant \frac{c_{1}\left(\mathscr{F}_{m} \mid C\right)}{\mathbf{r}}=\frac{\widetilde{d}_{m} c_{1}\left(\mathscr{F}_{m}\right)}{\mathbf{r}} \geqslant \frac{\widetilde{d}_{m}}{\mathbf{r}} .
$$

Комбинируя это с $(6.15)$, получаем $\delta_{A}\left(\left.E_{1}\right|_{l}\right) \geqslant \widetilde{d}_{m} / \mathbf{r}$, в частности,

$$
b_{1}:=\max _{l^{\prime} \in \mathscr{H}_{1}}\left\{\delta_{A}\left(E_{1} \mid l^{\prime}\right)\right\} \geqslant \frac{\widetilde{d}_{m}}{\mathbf{r}} .
$$

Если $\mathscr{F}_{m}$ существует для бесконечного числа значений $m$, то правая часть в последнем неравенстве стремится к бесконечности при $m \rightarrow \infty$, поскольку $\lim _{m \rightarrow \infty} \widetilde{d}_{m}=\infty$. Противоречие.

СлеДСтвиЕ 2. Для достаточно больиих $m \delta_{A}^{\text {gen }}\left(E_{m}\right) \leqslant \frac{1}{2} \mathbf{r}$.

Зафиксируем точку $x \in G_{m}$ и для любого $d \in \mathbb{Z}_{>0}$ рассмотрим локально замкнутое подмножество

$$
B_{a}^{0}(x):=\left\{l \in \pi_{1}^{-1}(x) \mid \delta\left(\left.E_{m}\right|_{l}\right)=a\right\}
$$

в $\pi_{1}^{-1}(x)$. Пусть $B_{a}(x)$ есть его замыкание в $\pi_{1}^{-1}(x)$. Полунепрерывность $\delta\left(\left.E_{m}\right|_{l}\right)$ влечет $B_{a}^{0}(x)=B_{a}(x) \backslash\left(\bigcup_{a^{\prime}>a} B_{a^{\prime}}(x)\right), a>0$. Обозначим

$$
\delta:=\delta_{A}\left(E_{m}\right), \quad \varkappa:=\varkappa_{A}\left(E_{m}\right) .
$$

Тогда

$$
B_{\delta}(x)=B_{\delta}^{0}(x)
$$

Кроме того, положим

$$
B_{\delta}^{\varkappa}(x):=\left\{l \in B_{\delta}(x) \mid \varkappa_{A}\left(\left.E_{m}\right|_{l}\right)=\varkappa\right\}
$$

и заметим, что $B_{\delta}^{\varkappa}(x)$ является замкнутым подмножеством в $B_{\delta}(x)$. Следующий результат принадлежит А.Н. Тюрину (см. [2; ч. $2, \S 2$, леммы 3 и 4]).

ЛЕмма 13. Если $B_{\delta}^{\varkappa}(x) \neq \varnothing$, mо $\operatorname{codim}_{\pi_{1}^{-1}(x)} B \leqslant \mathbf{r}(\mathbf{r}-1) \delta\left(E_{m}\right)$ для любой неприводимой компоненты $B$ в $B_{\delta}^{\varkappa}(x)$.

Поскольку $E_{m}$ самодвойственно, получаем, что $\delta\left(E_{m}\right)=2 \delta$, и, тем самым, лемма 13 влечет

$$
\operatorname{codim}_{\pi_{1}^{-1}(x)} B \leqslant 2 \mathbf{r}(\mathbf{r}-1) \delta
$$

при условии, что $B_{\delta}^{\varkappa}(x) \neq \varnothing$. 
Рассмотрим замкнутое подмножество

$$
W:=\left\{x \in G_{m} \mid B_{\delta_{A}^{\text {gen }}\left(E_{m}\right)}^{0}(x)=\varnothing\right\}
$$

в $G_{m}$ и положим

$$
G_{m}^{*}:=\left(G_{m} \backslash W\right) \cap G_{m}^{0},
$$

где $G_{m}^{0}$ было определено в (6.13) и (6.14). Ясно, что $W \cap l=\varnothing$ для общей прямой $l \subset G_{m}$, а следовательно, $G_{m}^{*}$ есть плотное открытое подмножество в $G_{m}$ и для любого $x \in G_{m}^{*}$ существует прямая $l \subset G_{m}$, проходящая через $x$, для которой $\delta_{A}\left(\left.E_{m}\right|_{l}\right)=\delta_{A}^{\text {gen }}\left(E_{m}\right)$.

Нам потребуется еще один результат А.Н. Тюрина. Из леммы 5 его статьи $[2 ;$ ч. $2, \S 2]$ непосредственно вытекает следующее.

СледСтвиЕ 3. Существует многочлен $F \in \mathbb{Q}\left[x_{1}, x_{2}\right]$ такой, что если $E$ самодвойственное векторное расслоение на $\mathbb{P}^{3}$ и $P$ - произвольная плоскость в $\mathbb{P}^{3}$, mo

$$
\delta_{A}\left(\left.E\right|_{l}\right) \leqslant F\left(\delta^{\text {gen }}(E), \chi\left(\left.E\right|_{P}\right)\right)
$$

для произвольной прямой $l \subset \mathbb{P}^{3}$.

Теперь зафиксируем точку $x \in G_{m}$, и пусть $K_{m}(x)$ - подмногообразие в $G_{m}$, заметаемое проективными подпространствами максимальной размерности в $G_{m}$, проходящими через $x$. Хорошо известно, что $K_{m}(x)$ является конусом над прямым произведением $\mathbb{P}^{i_{m}-1} \times \mathbb{P}^{n_{m}-i_{m}-1}$. Из следствия 3 вытекает, что для любой прямой $l \in p_{1}^{-1}(x)$ выполнено неравенство

$$
\delta_{A}\left(\left.E_{m}\right|_{l}\right) \leqslant F\left(\delta^{\text {gen }}\left(E_{m}\right), \chi\left(\left.E_{m}\right|_{P}\right)\right),
$$

где $F \in \mathbb{Q}\left[x_{1}, x_{2}\right]$ - некоторый многочлен и $P \subset K_{m}(x)$ - некоторая проективная плоскость. Класс плоскости $P$ в кольце Чжоу $A\left(G_{m}\right)$ совпадает с классом плоскости, содержащейся в проективном подпространстве одного из семейств $\mathbb{I}_{1}$ или $\mathbb{I}_{2}$ на $G_{m}$. Следовательно, в силу того, что $c_{1}\left(E_{m}\right)=0$, теорема Римана-Роха и следствие 1 влекут, что $\chi\left(\left.E\right|_{P}\right)$ совпадает с $\mathbf{r}-\lambda_{1}$ или $\mathbf{r}-\lambda_{2}$. Подставляя это в (6.19) и учитывая следствие 2, мы видим, что существует константа $\Delta$, не зависящая от $m$, ограничивающая $\delta_{A}\left(\left.E_{m}\right|_{l}\right)$ сверху для произвольной прямой $l \in p_{1}^{-1}(x)$ и произвольной точки $x \in G_{m}^{*}$.

Переходя от последовательности $\left(G_{m}, E_{m}\right)_{m \geqslant 1}$ к ее подходящей подпоследовательности $\left(G_{m^{\prime}}, E_{m^{\prime}}\right)_{m^{\prime} \geqslant 1}$ и заменяя исходную последовательность этой подпоследовательностью, мы получаем в силу (6.11), (6.12)-(6.14), леммы 13 и (6.18) следующий результат.

ПРеДЛОЖЕНИЕ. Существуют константы $\Delta, K u m_{0} \geqslant 1$ такие, что для любого $m \geqslant m_{0}$ существует плотное открытое подмножество $G_{m}^{*}$ в $G_{m}$ такое, что для любой точки $x \in G_{m}^{*}$ выполняются следующие утверждения.

(1) $\delta_{A}\left(\left.E_{m}\right|_{l}\right) \leqslant \Delta, \varkappa_{A}\left(\left.E_{m}\right|_{l}\right) \leqslant K$ для прочзвольной прямой $l \in B_{m}(x) u$

$$
\delta_{A}\left(\left.E_{m}\right|_{l}\right)=\Delta, \quad \varkappa_{A}\left(\left.E_{m}\right|_{l}\right)=K
$$

для некоторой прямой $l \in B_{m}(x)$. Поэтому $B_{\Delta}^{K}(x) \neq \varnothing u$

$$
\operatorname{codim}_{\pi_{1}^{-1}(x)} B \leqslant 2 \mathbf{r}(\mathbf{r}-1) \Delta
$$


для любой неприводимой компоненты $B$ множества $B_{\Delta}^{K}(x)$ в соответствии с леммой 13.

(2) Пусть $B_{\Delta}^{K}\left(E_{m}\right)^{*}:=\left(p_{\Delta, E_{m}}^{K}\right)^{-1}\left(G_{m}^{*}\right)$. Тогда $p_{\Delta, E_{m}}^{K}: B_{\Delta}^{K}\left(E_{m}\right)^{*} \rightarrow G_{m}^{*}$ является проективным сюргективным морфизмом таким, что

$$
\left(p_{\Delta, E_{m}}^{K}\right)^{-1}(x)=B_{\Delta}^{K}(x) .
$$

6.5. Завершающие утверждения. Нам остается доказать следующее утверждение.

ТеОрема 8. В условиях предложения из $n .6 .4$ положим $\Delta>0$. Тогда существует подпучок $\mathscr{F}_{m}$ в $E_{m}$ с $c_{1}\left(\mathscr{F}_{m}\right)>0$.

ДокАЗАтельство. Рассмотрим относительный грассманиан $g: G\left(K, E_{m}\right) \rightarrow$ $G_{m}$ со слоем $g^{-1}(x)=G\left(K,\left.E_{m}\right|_{x}\right)$ над $x \in G_{m}$. Положим $G\left(K, E_{m}\right)^{*}:=$ $g^{-1}\left(G_{m}^{*}\right)$. В соответствии с предложением из п. 6.4 для любой точки $(x, l) \in$ $B_{\Delta}^{K}\left(E_{m}\right)^{*}$ существует подрасслоение

$$
F(x, l) \simeq \mathscr{O}_{\mathbb{P}^{1}}(\Delta)^{K}
$$

в $\left.E\right|_{l}$. Это дает морфизм

$$
\Phi: B_{\Delta}^{K}\left(E_{m}\right)^{*} \rightarrow G\left(K, E_{m}\right)^{*},\left.\quad(x, l) \mapsto F(x, l)\right|_{x},
$$

который, очевидно, входит в коммутативную диаграмму

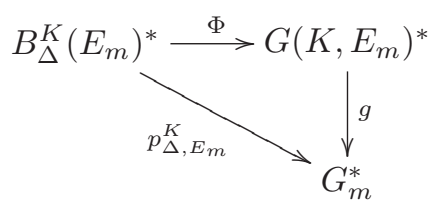

В остальной части доказательства мы предполагаем, что $i_{m} \geqslant 2$. Оставшийся случай $i_{m}=1$ есть случай скрученного инд-проективного пространства, и мы оставляем его читателю в качестве упражнения. Заметим, что (поскольку $\left.i_{m} \geqslant 2\right) G_{m}=G\left(i_{m}, V^{n_{m}}\right)$ входит в диаграмму (3.9) для $V=V^{n_{m}}, i=i_{m}-1$, и положим $p:=p_{i_{m}-1}, q:=q_{i_{m}-1}$; получим диаграмму

$$
G_{m} \stackrel{p}{\leftarrow} \mathrm{Fl}\left(i_{m}-1, i_{m}, V^{n_{m}}\right) \stackrel{q}{\rightarrow} G\left(i_{m}-1, V^{n_{m}}\right) .
$$

Зафиксируем подпространство $V_{0}^{n_{m}-1}$ в $V^{n_{m}}$ и положим $Y:=q^{-1}\left(G\left(i_{m}-1\right.\right.$, $\left.\left.V_{0}^{n_{m}-1}\right)\right)$. Проекция $\sigma:=\left.p\right|_{Y}: Y \rightarrow G_{m}$ есть не что иное как раздутие $G_{m}$ с центром в подмногообразии

$$
Z_{0}:=G\left(i_{m}, V_{0}^{n_{m}-1}\right), \quad \operatorname{codim}_{G_{m}} Z_{0}=i_{m} \geqslant 2 .
$$

Зафиксируем произвольную точку $x \in G_{m}^{*} \backslash Z_{0}$ и рассмотрим проективное подпространство

$$
\mathbb{P}_{x}^{n_{m}-i_{m}}:=\sigma\left(q^{-1}\left(q\left(\sigma^{-1}(x)\right)\right)\right) \subset G_{m},
$$

проходящее через $x$. Заметим, что слой $B_{\Delta}^{K}(x)=\left(p_{\Delta, E_{m}}^{K}\right)^{-1}(x)$ проекции

$$
p_{\Delta, E_{m}}^{K}: B_{\Delta}^{K}\left(E_{m}\right)^{*} \rightarrow G_{m}^{*}
$$


лежит в $p_{1}^{-1}(x)$. Далее, полагая

$$
\begin{gathered}
\mathbb{P}^{n_{m}-i_{m}-1}(x):=\left\{\mathbb{P}^{i_{m}} \text { принадлежит семейству } \mathbb{I}_{1} \mid x \in \mathbb{P}^{i_{m}}\right\}, \\
\mathbb{P}^{i_{m}-1}(x):=\left\{\mathbb{P}^{n_{m}-i_{m}} \text { принадлежит семейству } \mathbb{I}_{2} \mid x \in \mathbb{P}^{n_{m}-i_{m}}\right\},
\end{gathered}
$$

рассмотрим изоморфизм

$$
\mathbb{P}^{i_{m}-1}(x) \times \mathbb{P}^{n_{m}-i_{m}-1}(x) \stackrel{\simeq}{\rightarrow} \pi_{1}^{-1}(x), \quad\left(\mathbb{P}^{n_{m}-i_{m}}, \mathbb{P}^{i_{m}}\right) \mapsto l=\mathbb{P}^{n_{m}-i_{m}} \cap \mathbb{P}^{i_{m}}
$$

и проекции

$$
\mathbb{P}^{n_{m}-i_{m}-1}(x) \stackrel{\mathrm{pr}_{1}}{\longleftarrow} \pi_{1}^{-1}(x) \stackrel{\mathrm{pr}_{2}}{\longrightarrow} \mathbb{P}^{i_{m}-1}(x) .
$$

По построению морфизма $\sigma$ база $\mathbb{P}_{x}^{n_{m}-i_{m}-1}$ семейства прямых через точку $x$, лежащих в подпространстве $\mathbb{P}_{x}^{n_{m}-i_{m}}$, есть слой проекции $\pi_{1}^{-1}(x) \stackrel{\mathrm{pr}_{2}}{\longrightarrow} \mathbb{P}^{i_{m}-1}(x)$ над некоторой точкой, определяемой точкой $x$.

Рассмотрим замкнутое подмножество

$$
B_{\Delta, x}^{K}:=B_{\Delta}^{K}(x) \cap \mathbb{P}_{x}^{n_{m}-i_{m}-1}
$$

в $\mathbb{P}_{x}^{n_{m}-i_{m}-1}$. Предложение из п. 6.4 влечет

$$
\operatorname{codim}_{\mathbb{P}_{x}^{n} n_{m}^{-i} m-1} X \leqslant 2 \mathbf{r}(\mathbf{r}-1) \Delta
$$

для любой неприводимой компоненты $X$ в $B_{\Delta, x}^{K}$. Пусть

$$
N:=2 \mathbf{r}(\mathbf{r}-1) \Delta+1 \text {. }
$$

Возьмем произвольное проективное подпространство $\mathbb{P}^{N-1} \subset \mathbb{P}_{x}^{n_{m}-i_{m}-1}$, и пусть $\mathbb{P}^{N} \subset \mathbb{P}_{x}^{n_{m}-i_{m}}$ - подпространство, заметаемое прямыми из $\mathbb{P}^{N-1}$. Положим $E^{0}:=\left.E_{m}\right|_{\mathbb{P}^{N}}$. Тогда $\delta_{A}\left(E^{0}\right)=\Delta, \varkappa_{A}\left(E^{0}\right)=K$ по предложению из п. 6.4 и $c_{2}\left(E^{0}\right)=\lambda_{1}$ согласно (6.9). Вдобавок, сравнивая (5.61) с (6.27) и (6.20), получаем

$$
B_{\Delta}^{K}\left(E^{0}, x, \mathbb{P}^{N-1}\right)=B_{\Delta, x}^{K} \cap \mathbb{P}^{N-1},
$$

и $(6.28)$ и $(6.29)$ влекут

$$
\operatorname{deg} B_{\Delta}^{K}\left(E^{0}, x, \mathbb{P}^{N-1}\right)=\operatorname{deg} B_{\Delta, x}^{K}
$$

для общего выбора подпространства $\mathbb{P}^{N-1}$ в $\mathbb{P}_{x}^{n_{m}-i_{m}-1}$. Применяя теорему 7 к векторному расслоению $E^{0}$ с $\delta_{A}\left(E^{0}\right)=\Delta$ и $\varkappa_{A}\left(E^{0}\right)=K$, получаем

$$
\operatorname{deg} B_{\Delta}^{K}\left(E^{0}, x, \mathbb{P}^{N-1}\right) \leqslant d,
$$

где $d$ - не зависящая от $m$ константа. Таким образом,

$$
\operatorname{deg} B_{\Delta, x}^{K} \leqslant d .
$$

Далее, предположим, что $m$ достаточно велико, так что оценка (6.28) для любой неприводимой компоненты в $B_{\Delta, x}^{K}$ вместе с условием $\lim _{m \rightarrow \infty}\left(n_{m}-i_{m}\right)=\infty$ дают связность $B_{\Delta, x}^{K}$. Но тогда $\operatorname{deg} X \leqslant d$ в силу (6.30). Без ограничения общности мы можем считать, что $\mathbb{P}_{x}^{n_{m}-i_{m}-1}=\operatorname{Span} X$. Поэтому, применяя теорему $4 \mathrm{k} X$, получаем следующее утверждение: для достаточно большого $m$ 
любые две точки на $X$ можно соединить цепочкой подпространств $\mathbb{P}^{\mathbf{k}_{0}} \subset X$, где $\mathbf{k}_{0}>\operatorname{dim} G\left(K,\left.E_{m}\right|_{x}\right)$. Поэтому все такие подпространства $\mathbb{P}^{\mathbf{k}_{0}}$ отображаются при морфизме $\Phi$ в одну и ту же точку. Следовательно, $\Phi(X)$ - точка, а поскольку $B_{\Delta, x}^{K}$ связно, то $\Phi\left(B_{\Delta, x}^{K}\right)=\Phi(X)$. Это определяет морфизм

$$
G_{m}^{*} \backslash Z_{0} \rightarrow G\left(K,\left.E_{m}\right|_{x}\right), \quad x \mapsto \Phi\left(B_{\Delta, x}^{K}\right),
$$

а значит, задает подрасслоение $\mathscr{F}_{m}^{\prime}$ в $\left.E_{m}\right|_{G_{m}^{*} \backslash Z_{0}}$.

Следующая хорошо известная конструкция показывает, что $\mathscr{F}_{m}^{\prime}$ продолжается до подпучка $\mathscr{F}_{m}$ в $E_{m}$. Эпиморфизм локально свободных пучков

$$
\left.E_{m}^{\vee}\right|_{G_{m}^{*} \backslash Z_{0}} \rightarrow\left(\mathscr{F}_{m}^{\prime}\right)^{\vee}
$$

определяет композицию вложений

$$
\zeta: \mathbb{P}\left(\mathscr{F}_{m}^{\prime}\right) \hookrightarrow \mathbb{P}\left(\left.E_{m}\right|_{G_{m}^{*} \backslash Z_{0}}\right) \hookrightarrow \mathbb{P}\left(E_{m}\right) .
$$

Пусть $U$ - замыкание $\zeta\left(\mathbb{P}\left(\mathscr{F}_{m}^{\prime}\right)\right)$ в $\mathbb{P}\left(E_{m}\right)$. Положим $A:=\mathscr{O}_{\mathbb{P}\left(E_{m}\right) / G_{m}}(1)$, и пусть $\theta: \mathbb{P}\left(E_{m}\right) \rightarrow G_{m}-$ структурный морфизм. Применяя функтор $R \cdot \theta_{*}$ к точной тройке $\left.0 \rightarrow \mathscr{I}_{U, \mathbb{P}\left(E_{m}\right)} \otimes A \rightarrow A \rightarrow A\right|_{U} \rightarrow 0$, получаем точную последовательность $E_{m}^{\vee} \stackrel{\varepsilon}{\rightarrow} \theta_{*}\left(\left.A\right|_{U}\right) \rightarrow R^{1} \theta_{*}\left(\mathscr{I}_{U, \mathbb{P}\left(E_{m}\right)} \otimes A\right)$. Морфизм $\left.\varepsilon\right|_{G_{m}^{*} \backslash Z_{0}}$ является эпиморфизмом, а значит, $\varepsilon^{\vee}: \mathscr{F}_{m}:=\left(\theta_{*}\left(\left.A\right|_{U}\right)\right)^{\vee} \rightarrow E_{m}-$ мономорфизм и $\left.\mathscr{F}_{m}\right|_{G_{m}^{*} \backslash Z_{0}} \simeq \mathscr{F}_{m}^{\prime}$

Остается показать, что $c_{1}\left(\mathscr{F}_{m}\right)>0$. Согласно $(6.21)$, если $x \in G_{m}^{*} \backslash Z_{0}$, то для любой точки $\left(x, l_{0}\right) \in B_{\Delta, x}^{K}$ имеем подрасслоение $\mathscr{F}\left(x, l_{0}\right) \simeq \mathscr{O}_{\mathbb{P}^{1}}(\Delta)^{K}$ в $\left.E_{m}\right|_{l}$. Следовательно, $c_{1}\left(\mathscr{F}\left(x, l_{0}\right)\right)>0$, поскольку $\Delta>0$. Прямая $l_{0}$ лежит в проективном подпространстве $\mathbb{P}_{x}^{n_{m}-i_{m}}$ в $G_{m}$, определенном в $(6.26)$, поэтому определение $(6.27)$ показывает, что $\left(y, l_{0}\right) \in B_{\Delta, y}^{K}$ для любого $y$ в плотном открытом подмножестве $U:=l_{0} \cap\left(G_{m}^{*} \backslash Z_{0}\right)$ прямой $l_{0}$. Поэтому $\left.\mathscr{F}\left(x, l_{0}\right)\right|_{U}=\left.\mathscr{F}_{m}\right|_{U}$, а значит, $\mathscr{F}\left(x, l_{0}\right)$ изоморфен локально свободному факторпучку пучка $\left.\mathscr{F}_{m}\right|_{l_{0}}$, т.e. $\mathscr{F}\left(x, l_{0}\right) \simeq\left(\left.\mathscr{F}_{m}\right|_{l_{0}}\right) / \operatorname{Torsion}\left(\left.\mathscr{F}_{m}\right|_{l_{0}}\right)$.

Из того, что пучок $\mathscr{F}_{m}$ не имеет кручения как подпучок в $E_{m}$, следует, что $\operatorname{codim}_{G_{m}} \operatorname{Sing} \mathscr{F}_{m} \geqslant 2$, так что $\operatorname{codim}_{\mathrm{Fl}_{m}} \pi_{2}\left(\pi_{1}^{-1}\left(\operatorname{Sing} \mathscr{F}_{m}\right)\right) \geqslant 1$. Поэтому найдется такая гладкая аффинная кривая $C \subset \mathrm{Fl}_{m}$ с отмеченной точкой $c \in C$, что

$$
\pi_{1}\left(\pi_{2}^{-1}(c)\right)=l_{0}, \quad(C \backslash\{c\}) \subset\left(\mathrm{Fl}_{m} \backslash \pi_{2}\left(\pi_{1}^{-1}\left(\operatorname{Sing} \mathscr{F}_{m}\right)\right)\right) .
$$

Рассмотрим линейчатую поверхность $S:=\pi_{2}^{-1}(C) \stackrel{\pi_{2}}{\rightarrow} C$ и положим

$$
\begin{gathered}
\mathscr{F}_{S}:=\left(\left.\pi_{1}^{*} \mathscr{F}_{m}\right|_{S}\right) / \operatorname{Torsion}\left(\left.\pi_{1}^{*} \mathscr{F}_{m}\right|_{S}\right), \quad \widetilde{l}_{0}:=\pi_{2}^{-1}(c), \\
\mathscr{F}_{\widetilde{l}_{0}}:=\left(\left.\mathscr{F}_{S}\right|_{\tilde{l}_{0}}\right) / \operatorname{Torsion}\left(\left.\mathscr{F}_{S}\right|_{\widetilde{l}_{0}}\right), \quad \widetilde{l}_{t}:=\pi_{2}^{-1}(t), \quad t \in C \backslash\{c\} .
\end{gathered}
$$

Из условия $\pi_{1}\left(\pi_{2}^{-1}(c)\right)=l_{0}$ вытекает, что $\left.\pi_{1}\right|_{\widetilde{l}_{0}}: \widetilde{l}_{0} \rightarrow l_{0}$ - изоморфизм, а следовательно, $\left(\left.\pi_{1}\right|_{\widetilde{l}_{0}}\right)^{*} \mathscr{F}\left(x, l_{0}\right) \simeq \mathscr{F}_{\widetilde{l}_{0}}$. Поэтому

$$
c_{1}\left(\mathscr{F}\left(x, l_{0}\right)\right)=c_{1}\left(\mathscr{F}_{\widetilde{l}_{0}}\right) .
$$

А из того, что $\left.\mathscr{F}_{m}\right|_{\pi_{1}\left(\widetilde{l}_{t}\right)}$ локально свободен для $t \in C \backslash\{c\}$ в силу включения $(C \backslash\{c\}) \subset\left(\mathrm{Fl}_{m} \backslash \pi_{2}\left(\pi_{1}^{-1}\left(\operatorname{Sing} \mathscr{F}_{m}\right)\right)\right)$, следует, что

$$
c_{1}\left(\left.\mathscr{F}_{S}\right|_{\tilde{l}_{t}}\right)=c_{1}\left(\mathscr{F}_{m}\right), \quad t \in C \backslash\{c\} .
$$


Мы утверждаем, что

$$
c_{1}\left(\mathscr{F}_{\widetilde{l}_{0}}\right) \leqslant c_{1}\left(\left.\mathscr{F}_{S}\right|_{\widetilde{l}_{t}}\right), \quad t \in C \backslash\{c\} .
$$

Действительно, так как $\mathscr{F}_{S}$ не имеет кручения, то, используя фильтрацию пучка $\mathscr{F}_{S}$ с последовательными факторами ранга 1 без кручения и удаляя, если нужно, конечное число точек из $C \backslash\{c\}$, мы сведем доказательство неравенства (6.33) к случаю, когда $\mathrm{rk} \mathscr{F}_{S}=1$. Тогда $\mathscr{F}_{S} \simeq \mathscr{I}_{Y, S} \otimes L$ для некоторого линейного расслоения $L$ на $S$ и некоторой подсхемы $Y$ в $S$ размерности $\leqslant 0$. Рассмотрим схему $Y_{0}:=Y \cap \widetilde{l}_{0}$ длины $\chi\left(\mathscr{O}_{Y_{0}}\right) \geqslant 0$ с носителем в $\widetilde{l}_{0}$. Тогда $\left.\mathscr{F}_{\widetilde{l}_{0}} \simeq L\right|_{\widetilde{l}_{0}}\left(-\chi\left(\mathscr{O}_{Y_{0}}\right)\right)$, следовательно,

$$
\begin{aligned}
c_{1}\left(\mathscr{F}_{\widetilde{l}_{0}}\right) & =c_{1}\left(\left.L\right|_{\widetilde{l}_{0}}\right)-\chi\left(\mathscr{O}_{Y_{0}}\right)=c_{1}\left(\left.L\right|_{\widetilde{l}_{t}}\right)-\chi\left(\mathscr{O}_{Y_{0}}\right) \\
& =c_{1}\left(\left.\mathscr{F}_{S}\right|_{\widetilde{l}_{t}}\right)-\chi\left(\mathscr{O}_{Y_{0}}\right) \leqslant c_{1}\left(\left.\mathscr{F}_{S}\right|_{\widetilde{l}_{t}}\right), \quad t \in C \backslash\{c\} .
\end{aligned}
$$

Наконец, $(6.31)-(6.33)$ влекут $c_{1}\left(\mathscr{F}_{m}\right) \geqslant c_{1}\left(\mathscr{F}\left(x, l_{0}\right)\right)>0$. Теорема доказана.

СлеДСтвиЕ 4. Для всех $m>0 E_{m}$ есть тривиальное векторное расслоение на $G_{m}$.

ДокАЗАтельство. Если для достаточно больших $m$ имеет место неравенство $\Delta>0$, то теорема 8 противоречит лемме 12 . Следовательно, $\Delta=0$. Покажем, что это равенство влечет тривиальность расслоения $E_{m}$.

Рассмотрим диаграмму (6.24). Заметим, что для любой точки $x \in G_{m}$ проективное подпространство $\mathbb{P}^{i_{m}-1}(x)$ в $G\left(i_{m}-1, V^{n_{m}}\right)$, введенное в доказательстве теоремы 8 , совпадает с $q\left(p^{-1}(x)\right)$. Аналогично, $\mathbb{P}^{n_{m}-i_{m}}(y):=p\left(q^{-1}(y)\right)$ есть проективное подпространство в $G_{m}$ для $y \in G\left(i_{m}-1, V^{n_{m}}\right)$. Более того, легко видеть, что конус $K(x):=\bigcup_{y \in \mathbb{P}_{m}-1}(x) \mathbb{P}^{n_{m}-i_{m}}(y)$ с вершиной в $x$, рассматриваемый как приведенная подсхема в $G_{m}$, имеет то же самое касательное пространство по Зарискому в точке $x$, что и $G_{m}$ :

$$
T_{x} K(x)=T_{x} G_{m} .
$$

Поскольку $\Delta=0$, в обозначениях доказательства теоремы 8 имеем $B_{\Delta}^{K}(x)=$ $\pi^{-1}(x)$ и $K=\mathbf{r}$ для любой точки $x \in G_{m}^{*}$, т.е. расслоение $\left.E_{m}\right|_{l}$ тривиально для любой проективной прямой $l \subset G_{m}$, проходящей через $x$. Отсюда следует, что расслоение $\left.E_{m}\right|_{\mathbb{P}_{m}-i_{m}}(y)$ тривиально для любой точки $y \in \mathbb{P}^{i_{m}-1}(x)$ (см., например, [15; ч. I, теорема 3.2.1]), и, следовательно, $\mathbb{P}^{n_{m}-i_{m}}(y) \subset G_{m}^{*}$.

Мы утверждаем, что $G_{m}^{*}=G_{m}$. В самом деле, если $G_{m}^{*} \neq G_{m}$, то для любой точки $x \in\left(G_{m} \backslash G_{m}^{*}\right)$ и любой точки $y \in \mathbb{P}^{i_{m}-1}(x)$ имеем $\mathbb{P}^{n_{m}-i_{m}}(y) \subset\left(G_{m} \backslash G_{m}^{*}\right)$. Следовательно, $K(x) \subset\left(G_{m} \backslash G_{m}^{*}\right)$ и в силу $(6.34)$

$$
T_{x} G_{m}=T_{x} K(x) \subset T_{x}\left(G_{m} \backslash G_{m}^{*}\right),
$$

где мы рассматриваем $\left(G_{m} \backslash G_{m}^{*}\right)$ как приведенную подсхему в $G_{m}$. Отсюда $\left(G_{m} \backslash G_{m}^{*}\right)=G_{m}$ вопреки тому, что $G_{m}^{*}$ есть плотное открытое подмножество в $G_{m}$.

Мы показали, что $G_{m}^{*}=G_{m}$ для достаточно большого $m$. Поскольку $\Delta=0$, это означает, что $\left.E_{m}\right|_{l}$ тривиально для любой прямой $l$ в $G_{m}$. В силу [7; предложение 1.4.1] этого достаточно для тривиальности расслоения $E_{m}$ для достаточно больших $m$, а значит, и для всех $m>0$. 


\section{Список литературы}

[1] W. Barth, A. Van de Ven, "On the geometry in codimension 2 of Grassmann manifolds", Classification of algebraic varieties and compact complex manifolds, Lecture Notes in Math., 412, Springer-Verlag, Berlin, 1974, 1-35.

[2] А.Н. Тюрин, "Конечномерные расслоения на бесконечомерных многообразиях", Изв. АН СССР. Сер. матем., 40:6 (1976), 1248-1268; англ. пер.: А. N. Tjurin, "Vector bundles of finite rank over infinite varieties", Math. USSR-Izv., 10:6 (1976), 1187-1204.

[3] E. Sato, "On the decomposability of infinitely extendable vector bundles on projective spaces and Grassmann varieties", J. Math. Kyoto Univ., 17:1 (1977), 127-150.

[4] E. Sato, "On infinitely extendable vector bundles on G/P", J. Math. Kyoto Univ., 19:1 (1979), 171-189.

[5] J. Donin, I. Penkov, "Finite rank vector bundles on inductive limits of grassmannians", Int. Math. Res. Not., 34 (2003), 1871-1887.

[6] I. Coandă, G. Trautmann, "The splitting criterion of Kempf and the Babylonian tower theorem", Comm. Algebra, 34:7 (2006), 2485-2488.

[7] I. Penkov, A.S. Tikhomirov, "Rank-2 vector bundles on ind-Grassmannians", Algebra, arithmetic, and geometry: in honor of Yu. I. Manin, v. II, Progr. Math., 270, Birkhäuser, Boston-Basel-Berlin, 2009, 555-572.

[8] Р. Хартсхорн, Алгебраическал геометрия, Мир, М., 1981; пер. с англ.: R. Hartshorne, Algebraic geometry, Grad. Texts in Math., 52, Springer-Verlag, New YorkHeidelberg-Berlin, 1977.

[9] A. B. Altman, S. L. Kleiman, "Foundations of the theory of Fano schemes", Compositio Math., 34:1 (1977), 3-47.

[10] R. Braun, S. Müller-Stach, "Effective bounds for Nori's connectivity theorem", Higher dimensional complex varieties (Trento, Italy, 1994), de Gruyter, Berlin, 1996, 83-88.

[11] R. Lazarsfeld, Positivity in algebraic geometry, v. I: Classical setting: line bundles and linear series, Ergeb. Math. Grenzgeb. (3), 48, Springer-Verlag, Berlin, 2004; v. II: Positivity for vector bundles, and multiplier ideals, Ergeb. Math. Grenzgeb. (3), 49, Springer-Verlag, Berlin, 2004.

[12] У. Фултон, Теория пересечений, Мир, М., 1989; пер. с англ.: W. Fulton, Intersection theory, Ergeb. Math. Grenzgeb. (3), 2, Springer-Verlag, Berlin, 1984.

[13] D. Huybrechts, M. Lehn, The geometry of moduli spaces of sheaves, Aspects Math., 31, Vieweg \& Sohn, Braunschweig, 1997.

[14] D. Eisenbud, Commutative algebra. With a view toward algebraic geometry, Grad. Texts in Math., 150, Springer-Verlag, New York, 1995.

[15] Ch. Okonek, M. Schneider, H. Spindler, Vector bundles on complex projective spaces, Progr. Math., 3, Birkhäuser, Boston-Basel-Stuttgart, 1980.

[16] S.A. Strømme, "On parametrized rational curves in Grassmann varieties", Space curves (Rocca di Papa, 1985), Lecture Notes in Math., 1266, Springer-Verlag, 1987, $251-272$.

И. Б. Пенков (I. В. Penkov)

School of Engineering and Science, Jacobs University Bremen, Germany

E-mail: i.penkov@jacobs-university.de
Поступила в редакцию 08.07.2009 и 19.07.2010

\section{A. С. Тихомиров (A.S. Tikhomirov)}

Ярославский государственный педагогический университет им. К. Д. Ушинского

E-mail: astikhomirov@mail.ru 